\title{
Atratores para equações de reação-difusão em domínios arbitrários
}

\author{
Henrique Barbosa da Costa
}




\title{
Atratores para equações de reação-difusão em domínios arbitrários
}

\author{
Henrique Barbosa da Costa
}

Orientadora: Profa. Dra. Maria do Carmo Carbinatto

Dissertação apresentada ao Instituto de Ciências Matemáticas e de Computação - ICMC-USP, como parte dos requisitos para obtenção do título de Mestre em Ciências - Matemática . VERSÃO REVISADA

USP - São Carlos

Junho de 2012 
Ficha catalográfica elaborada pela Biblioteca Prof. Achille Bassi e Seção Técnica de Informática, ICMC/USP, com os dados fornecidos pelo(a) autor(a)

Costa, Henrique Barbosa da
Atratores para equaçóes de reação-difusão em
domínios arbitrários / Henrique Barbosa da Costa;
orientadora Maria do Carmo Carbinatto. -- São
Carlos, 2012 .
86 p.
Dissertação (Mestrado - Programa de Pós-Graduação en
Matemática)-- Instituto de Ciências Matemáticas e
de Computação, Universidade de São Paulo, 2012.
1. Atratores globais. 2. Equações parabólicas. 3.
Equaços de reação-difusão. 4. Estimativas de
truncamento. I. Carbinatto, Maria do Carmo, orient.
II. Título.


“E aqueles que foram vistos dançando foram julgados insanos por aqueles que não podiam escutar a música”. Friedrich Nietzsche 



\section{Agradecimentos}

Agradeço a minha família pelas horas passadas em casa, que sempre foram confortantes, independente do momento. Em especial à minha mãe, que sempre me guiou e aconselhou nas encruzilhadas que passamos. Fico feliz em dizer que ela cumpriu bem o seu papel, que segundo ela mesma é "criar asas"para que possa ficar tão longe da família e não me sentir triste e "manter raízes"para que sempre saiba onde procurar conselhos e se sentir em casa. E agradeço ao meu pai pelos genes, afinal, a culpa por seguir esta profissão tem que ser de alguém.

Agradeço aos meus irmãos. A quem eu deveria servir de exemplo, por ser o primogênito, mas que, às vezes, sinto que são mais exemplos para mim do que sou a eles.

Agradeço a minha namorada, que sempre fez tão bem pra mim, apesar da distância. Por me apoiar, sustentar e me amar apesar de todos pesares. Eu sei que é difícil, mas se fosse fácil, que graça teria?

Agradeço aos meus amigos. Os amigos de Sete Lagoas por serem meu escape. Como dizem, os amigos são a família que nos deixam escolher, e creio que escolhi meus irmãos muito bem. E os amigos de São Carlos que tornaram minha moradia aqui quase nada complicada, fazendo estudar matemática parecer fácil e divertido (talvez tenha exagerado um pouco aqui).

Agradeço à minha orientadora, Maria do Carmo Carbinatto, pela paciência, devoção e cuidado. Por toda a orientação, que não teria como ser melhor. Pelo fim dessa jornada me sinto orgulhoso em poder chama-la de amiga.

Agradeço, por fim, à FAPESP pela confiança e apoio financeiro. 



\section{Resumo}

Neste trabalho estudamos a dinâmica assintótica de uma classe de equações diferenciais de reação-difusão definidas em abertos de $\mathbb{R}^{3}$ arbitrários, limitados ou não, com condições de fronteira de Dirichlet. Utilizando a técnica de estimativas de truncamento, como nos artigos de Prizzi e Rybakowski, mostramos a existência de atratores globais.

Palavras-chave: Atratores globais, equações parabólicas, equações de reação-difusão, estimativas de truncamento. 



\section{Abstract}

In this work we study the asymptotic behavior of a class of semilinear reaction-diffusion equations defined on an arbitrary open set of $\mathbb{R}^{3}$, bounded or not, with Dirichlet boundary conditions. Using the tail-estimates technic based on papers of Prizzi and Rybakowski, we prove existence of global attractors.

Key words: Global attractors, parabolic equations, reaction-diffusion equations, tail-estimates. 



\section{Sumário}

Introdução

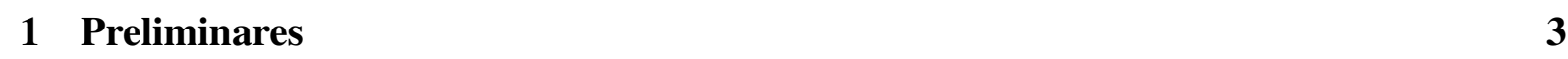

1.1 Medidas espectrais $\ldots \ldots \ldots \ldots \ldots \ldots$

1.2 Operadores de Nemytskiil $\ldots \ldots \ldots \ldots$. . . . . . . . . . . . . 7

1.3 Medida de não-compacidade de Kuratowski $\ldots \ldots \ldots$. . . . . . . . . . . . . 9

1.4 Operadores $m$-dissipativos $\ldots \ldots \ldots \ldots$

1.5 Operadores setoriais $\ldots \ldots \ldots \ldots \ldots \ldots$

1.6 Semifluxos e atratores globais $\ldots \ldots \ldots \ldots \ldots \ldots$

1.7 Equações diferenciais parabólicas $\ldots \ldots \ldots \ldots . \ldots . \ldots . \ldots . \ldots 24$

\begin{tabular}{|lll}
\hline 2 & O problema linear & 31
\end{tabular}

$2.1 \quad$ Um resultado de análise funcional $\ldots \ldots \ldots$. . . . . . . . . . . . 31

2.2 Resultados auxiliares sobre espaços de potências fracionárias . . . . . . . . . . 34

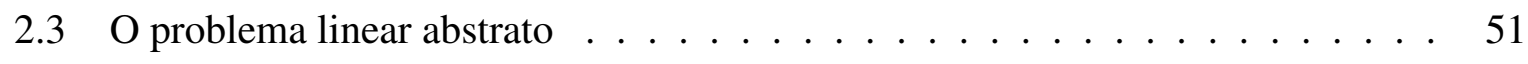

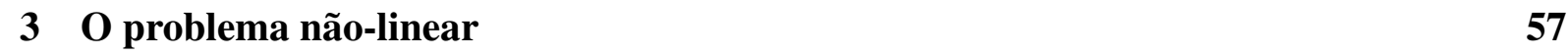

3.1 Estimativas não-lineares . . . . . . . . . . . . . . . . . . 57 
3.2 A equação de evolução abstrata $\ldots \ldots \ldots \ldots$. . . . . . . . . . . . 65

4 Dinâmica assintótica $\quad 69$

$4.1 \quad$ Equações parabólicas semilineares $\ldots \ldots$. . . . . . . . . . . . . . . 69

4.2 Construção da função de Lyapunov . . . . . . . . . . . . . . . . . . . . . 73

4.3 Estimativas de truncamento . . . . . . . . . . . . . . . . . . . 76

4.4 Compacidade assintótica e existência de atrator $\ldots \ldots \ldots$. . . . . . . . . . 80

5 Considerações finais $\quad 83$

\begin{tabular}{ll}
\hline Referências bibliográficas & 85
\end{tabular} 


\section{Introdução}

A teoria de sistemas dinâmicos descreve fenômenos que evoluem com o tempo. Dessa forma, sistemas dinâmicos servem de modelo para várias áreas das ciências aplicadas. Uma questão que podemos colocar é entender o compartamento assintótico de um determinado sistema dinâmico. Neste contexto, podemos investigar a existência de conjuntos invariantes com determinadas propriedades ou, em particular, a existência de atratores globais. O papel dos atratores globais está diretamente relacionado com o estudo da dinâmica assintótica dos sistemas dinâmicos. A existência de um atrator global nos fornece a garantia de que o fenômeno se aproxima de um padrão no futuro.

Neste trabalho, estudamos uma classe de equações diferenciais parciais parabólicas e apresentamos condições para existência de um atrator global. Mais especificamente, consideramos a seguinte equação de reação-difusão semilinear

$$
\begin{array}{ll}
u_{t}+\beta(x) u-\sum_{i, j} \partial_{i}\left(a_{i j}(x) \partial_{j} u\right)=f(x, u), & t \geq 0, x \in \Omega \\
u(x, t)=0, & t \geq 0, x \in \partial \Omega
\end{array}
$$

onde $\Omega$ é um aberto arbitrário em $\mathbb{R}^{N}$ (limitado ou não), $\beta: \Omega \rightarrow \mathbb{R}$ e $f: \Omega \times \mathbb{R} \rightarrow \mathbb{R}$ são funções dadas e $\mathrm{L} u:=\sum_{i, j} \partial_{i}\left(a_{i j}(x) \partial_{j} u\right)$ é o operador diferencial de segunda ordem na forma divergente.

Existe uma vasta literatura tratando da existência de atratores para equações de reaçãodifusão em domínios limitados (ver, por exemplo, os livros [14], [2], [7], [17] e [23]). Neste caso, a compacidade assintótica das soluções é obtida da compacidade da inclusão de Sobolev $H^{1}(\Omega) \subset L^{2}(\Omega)$.

Para caso de domínios não limitados, esta inclusão não é compacta e necessitamos de outras ferramentas para obter a propriedade da compacidade assintótica das soluções. Nos trabalhos [3], [1], [21], [24] os autores mostram a existência de atrator global para uma equação de 
reação-difusão definida em um domínio não limitado. O objetivo deste trabalho é apresentar o trabalho desenvolvido em [21].

Em [21], os autores mostram a existência de atrator global para a equação parabólica $E R D$, definida em um domínio $\Omega$ não limitado de $\mathbb{R}^{3}$ (isto é, $N=3$ ) sem supor qualquer condição de regularidade na fronteira $\partial \Omega$ de $\Omega$ ou nas funções $a_{i j}(\cdot)$. No trabalho, os autores exploram da técnica de estimativas de truncamento desenvolvida por Wang em [24] e o fato de que a equação do calor admite um funcional de Lyapunov.

Observamos que sem hipóteses de regularidade sobre $\partial \Omega$ e em $a_{i j}(\cdot)$ não é possível estudar $E R D$ em $L^{q}(\Omega)$ com $q \neq 2$. Isso se dá porque não podemos usar a teoria de regularidade das equações elípticas para caracterizar os espaços de potências fracionárias gerados pelo operador $-\mathrm{L}+\beta(x) I$. Contudo, de modo a trabalhar em $L^{2}(\Omega)$, devemos impor condições de crescimento em $f$. No caso particular em que $N=3$ o expoente crítico é $\bar{\rho}=5$.

Para descrever o trabalho [21] organizamos a apresentação como segue. No Capítulo 1 enumeramos alguns conhecimentos preliminares importantes que foram estudados para o desenvolvimento do trabalho.

No Capítulo 2 apresentamos as Hipóteses (HL1), (HL2) e (HL3). Com estas hipóteses o operador $u \mapsto-\mathrm{L} u+\beta(x) u$ define um operador positivo auto-adjunto $\mathbf{A}: D(\mathbf{A}) \subset X \rightarrow X$, onde $X=L^{2}(\Omega)$. Apresentamos as propriedades importantes do operador $\mathbf{A}$ e construímos uma família de operadores auto-adjuntos $\mathbf{A}_{(\alpha)}, \alpha \in \mathbb{R}$ tal que $\mathbf{A}_{(\alpha)}: X^{\alpha} \rightarrow X^{\alpha-1}$.

No Capítulo 3 apresentamos a Hipótese (HNL) e determinamos um $\alpha \in[0,1)$ tal que a partir uma função $f$ satisfazendo Hipótese (HNL) obtemos um operador de Nemytskiǔf : $H_{0}^{1}(\Omega) \rightarrow$ $X^{-\alpha}$. Munido dos resultados sobre equações parabólicas apresentados no Capítulo 1 obtemos que $E R D$ gera um semifluxo global $\pi$ em $H_{0}^{1}(\Omega)$.

Finalmente, no Capítulo 4, demonstramos o principal resultado deste trabalho que diz que assumindo as Hipóteses (HL1), (HL2), (HL3) e (HNL), o semifluxo $\pi$ possui um atrator global (ver Teorema 4.4.2). 


\section{Capítulo}

\section{1}

\section{Preliminares}

Neste capítulo apresentamos os diversos conceitos e resultados básicos estudados.

\subsection{Medidas espectrais}

As referências [10] e [16] foram consultadas para esta seção.

A teoria espectral para operadores fechados tem como objetivo representar operadores lineares fechados de um modo mais simples. Se $X$ é um espaço de Banach e $A: D(A) \subset X \rightarrow X$ é um operador linear fechado, o elemento principal dessa teoria é o espectro do operador $A$, $\sigma(A)$

Outra consequência importante da teoria espectral, além de escrever o operador de forma mais simples, é o cálculo operacional. Com o cálculo operacional podemos "aplicar" funções a operadores lineares. Isso é, se $A$ é um operador linear fechado e $f: \mathbb{R} \rightarrow \mathbb{R}$ tem certas propriedades, poderemos definir $f(A)$ que será um operador linear limitado em $\mathscr{L}(X)=\{B: X \rightarrow X \mid$ $B$ é linear e limitado $\}$.

As medidas espectrais ou famílias espectrais se encontram nesse contexto. Quando $X$ é um espaço de Hilbert e $A$ é um operador auto-adjunto podemos definir uma família de subespaços de $X$ que se relaciona de modo biunívoco com o operador $A$. É possível também aplicar o cálculo operacional para estas famílias e realizar o cálculo de funções de operadores. No caso particular estudado aqui, o cálculo operacional será importante na geração das famílias de espaços de 
potências fracionárias de $X$.

Dessa forma, no que segue $X$ denota um espaço de Hilbert com produto interno $\langle\cdot, \cdot\rangle_{X}$. Vamos então, definir de uma forma mais abstrata, o conceito de família espectral.

Seja $\left\{E_{\lambda}\right\}_{\lambda \in \mathbb{R}}$ uma família de projeções ortogonais em $X$. Dado $x \in X$ definimos

$$
\begin{aligned}
E_{-\infty} x & =\lim _{\lambda \rightarrow-\infty} E_{\lambda} x, \\
E_{+\infty} x & =\lim _{\lambda \rightarrow \infty} E_{\lambda} x \mathrm{e} \\
E_{\lambda+0} x & =\lim _{\varepsilon \rightarrow 0^{+}} E_{\lambda+\varepsilon} x .
\end{aligned}
$$

Definição 1.1.1. Uma família $\left\{E_{\lambda}\right\}_{\lambda \in \mathbb{R}}$ de projeções ortogonais em X é chamada uma família espectral ou resolução da identidade se satisfaz as condições:

(i) $E_{\lambda} \circ E_{\mu}=E_{\min \{\lambda, \mu\}}$, para todo $\lambda, \mu \in \mathbb{R}$.

(ii) $E_{-\infty}=0$ e $E_{+\infty}=I$.

(iii) $E_{\lambda+0}=E_{\lambda}$ para todo $\lambda \in \mathbb{R}$.

Observamos que os limites acima são tomados na norma de $X$.

Proposição 1.1.2. Seja $\left\{E_{\lambda}\right\}_{\lambda \in \mathbb{R}}$ uma família espectral em $X$. Então para todo $u, v \in X a$ função

$$
\lambda \in \mathbb{R} \mapsto\left\langle E_{\lambda} u, v\right\rangle_{X} \in \mathbb{R}
$$

é uma função de variação limitada em todo intervalo limitado da reta. Além disso, sua variação total $V(\lambda ; u, v)$ satisfaz

$$
V(\lambda ; u, v) \leq|u||v|, \text { para todo } u, v \in X \text { e } \lambda \in \mathbb{R}
$$

Sejam $[\alpha, \beta] \subset \mathbb{R}$ um intervalo fechado de $\mathbb{R}, f: \mathbb{R} \rightarrow \mathbb{C}$ uma função e $u \in X$. Considere uma partição $\pi=\left\{\alpha=\lambda_{1}, \lambda_{2}, \ldots, \lambda_{n}=\beta\right\}$ do intervalo $[\alpha, \beta]$. Defina a norma de $\pi$ por

$$
\|\pi\|=\max _{j \in\{1, \ldots, n\}}\left|\lambda_{j+1}-\lambda_{j}\right|
$$


Para cada $j \in\{1, \ldots, n\}$ seja $\lambda_{j}^{\prime} \in\left(\lambda_{j}, \lambda_{j+1}\right]$. A soma

$$
\sum_{j=1}^{n} f\left(\lambda_{j}^{\prime}\right)\left(E_{\lambda_{j+1}}-E_{\lambda_{j}}\right) u
$$

é chamada a soma de Riemann da função $f$ com relação à partição $\pi$.

Proposição 1.1.3. Com a notação introduzida acima, suponha que $f: \mathbb{R} \rightarrow \mathbb{C}$ seja uma função contínua. O limite forte em $X$

$$
\lim _{\pi \rightarrow 0} \sum_{j=1}^{n} f\left(\lambda_{j}^{\prime}\right)\left(E_{\lambda_{j+1}}-E_{\lambda_{j}}\right) u
$$

existe, para qualquer que seja a escolha de $\lambda_{j}^{\prime}$ no intervalo $\left(\lambda_{j}, \lambda_{j+1}\right], j \in\{1, \ldots, n\}$.

Denotamos o limite (1.1.2) por

$$
\int_{\alpha}^{\beta} f(\lambda) d E_{\lambda} u
$$

Se $u \in X$ e $f: \mathbb{R} \rightarrow \mathbb{C}$ é uma função contínua tal que os limites (forte em $X$ )

$$
\lim _{\alpha \rightarrow-\infty} \int_{\alpha}^{\beta} f(\lambda) d E_{\lambda} u \text { e } \lim _{\beta \rightarrow \infty} \int_{\alpha}^{\beta} f(\lambda) d E_{\lambda} u
$$

existem, dizemos que a integral $\int_{-\infty}^{+\infty} f(\lambda) d E_{\lambda} u$ existe.

Teorema 1.1.4. Sejam $u \in X$ e $f: \mathbb{R} \rightarrow \mathbb{C}$ uma função contínua. As seguintes afirmativas são equivalentes:

(i) $\int_{-\infty}^{+\infty} f(\lambda) d E_{\lambda}$ u existe.

(ii) $\int_{-\infty}^{+\infty}|f(\lambda)|^{2} d\left|E_{\lambda} u\right|^{2}<\infty$

(iii) A aplicação $v \mapsto F(v):=\int_{-\infty}^{+\infty} f(\lambda) d\left\langle E_{\lambda} v, u\right\rangle_{X}$ é um funcional linear contínuo.

Teorema 1.1.5. Seja $f: \mathbb{R} \rightarrow \mathbb{R}$ uma função contínua. Defina o seguinte subconjunto de $X$ :

$$
D=\left\{\left.u \in X\left|\int_{-\infty}^{+\infty}\right| f(\lambda)\right|^{2} d\left|E_{\lambda} u\right|^{2}<\infty\right\}
$$


Então Dé denso em X. Definimos operador por

$$
\langle T u, v\rangle_{X}=\int_{-\infty}^{+\infty} f(\lambda) d\left\langle E_{\lambda} u, v\right\rangle_{X}, u \in D e v \in X
$$

Então $T$ é um operador auto-adjunto em $X$ com domínio $D(T)=D$.

Com a notação do Teorema 1.1.5, para o caso particular em que $f(\lambda)=\lambda, \lambda \in \mathbb{R}$, definimos

$$
\langle A u, v\rangle_{X}=\int_{-\infty}^{+\infty} \lambda d\left\langle E_{\lambda} u, v\right\rangle_{X}, \text { para } u \in D(A) \subset X \text { e } v \in X
$$

onde

$$
D(A)=\left\{\left.u \in X\left|\int_{-\infty}^{+\infty} \lambda^{2} d\right| E_{\lambda} x\right|^{2}<\infty\right\} .
$$

No que segue o operador $A$ será denotado, simbolicamente, por

$$
A=\int_{-\infty}^{+\infty} \lambda d E_{\lambda}
$$

e chamamos (1.1.4) a representação espectral do operador auto-adjunto A no espaço de Hilbert $X$.

Reciprocamente, se $A: D(A) \subset X \rightarrow X$ é um operador linear auto-adjunto, podemos definir uma família espectral $\left\{E_{\lambda}\right\}_{\lambda \in \mathbb{R}}$ correspondente ao operador $A$. Descrevemos esta conexão no Teorema Espectral a seguir.

Teorema 1.1.6 (Teorema Espectral). Sejam X um espaço de Hilbert complexo e $A: D(A) \subset X \rightarrow$ $X$ um operador auto-adjunto. Então existe uma única família espectral $\left\{E_{\lambda}\right\}_{\lambda \in \mathbb{R}}$ associada ao operador A. Além disso para cada $\lambda \in \mathbb{R} e \xi \in \mathbb{C} / \mathbb{R}$ temos

$$
\left\langle(\xi-A)^{-1} u, v\right\rangle_{X}=\int_{-\infty}^{+\infty} \frac{1}{\xi-\lambda} d\left\langle E_{\lambda} u, v\right\rangle_{X} \text {, para todo } u, v \in X
$$

Finalmente obtemos

Corolário 1.1.7. Sejam X um espaço de Hilbert e A um operador auto-adjunto em X. Existe 
uma única família espectral $\left\{E_{\lambda}\right\}_{\lambda \in \mathbb{R}}$ tal que

$$
\begin{aligned}
\langle A u, v\rangle_{X} & =\int_{\mathbb{R}} \lambda d\left\langle E_{\lambda} u, v\right\rangle_{X}, \\
A u & =\int_{\mathbb{R}} \lambda d E_{\lambda} u .
\end{aligned}
$$

Concluímos observando que existe uma correspondência entre operadores auto-adjuntos no espaço de Hilbert $X$ e famílias espectrais. Dessa maneira, analogamente ao Teorema 1.1.4, podemos definir os operadores $f(A)$. Se $f$ for uma função em $\mathbb{R}$ a valores complexos limitada em $\sigma(A)$, o espectro de $A$, então $f(A)$ é um operador linear limitado em $X$ e, ainda,

$$
\|f(A)\| \leq \sup _{\lambda \in \sigma(A)}|f(\lambda)|
$$

Escrevemos, anolagamente à equação (1.1.4 e ao Corolário 1.1.7.

$$
\begin{gathered}
f(A)=\int_{-\infty}^{+\infty} f(\lambda) d E_{\lambda} . \\
f(A) u=\int_{-\infty}^{+\infty} f(\lambda) d E_{\lambda} u, \text { para todo } u \in X .
\end{gathered}
$$

\subsection{Operadores de Nemytskiǐ}

Para os conceitos apresentados e demonstrações dos resultados enunciados, sugerimos [12].

Sejam $N \geq 1$ um número natural e $\Omega \subset \mathbb{R}^{N}$ um subconjunto aberto de $\mathbb{R}^{N}$. Dizemos que $f: \Omega \times \mathbb{R} \rightarrow \mathbb{R}$ é uma função de Carathéodory se

1) para cada $s \in \mathbb{R}$, a função $\Omega \ni x \mapsto f(x, s)$ é (Lebesgue) mensurável e

2) para q.t.p. $x \in \Omega$, a função $\mathbb{R} \ni s \mapsto f(x, s)$ é contínua em $\mathbb{R}$.

Denotemos por $\mathscr{M}$ o conjunto das funções mensuráveis de $\Omega$ em $\mathbb{R}$.

Teorema 1.2.1. Se $f: \Omega \times \mathbb{R} \rightarrow \mathbb{R}$ é uma função de Carathéodory, então a função $\Omega \ni x \mapsto$ $f(x, u(x))$ é mensurável para todo $u \in \mathscr{M}$. 
Portanto, uma função de Carathéodory $f$ define uma aplicação $\widehat{f}: \mathscr{M} \rightarrow \mathscr{M}$, chamada aplicação de Nemytskiǔ.

Muitos resultados importantes requerem que o subconjunto $\Omega$ de $\mathbb{R}^{N}$ seja limitado e como não estamos nos restringindo a apenas este caso enumeramos apenas alguns resultados que não necessitem desta restrição em $\Omega$.

Teorema 1.2.2. Seja $\Omega$ um subconjunto aberto de $\mathbb{R}^{N}$ e seja $f: \Omega \times \mathbb{R} \rightarrow \mathbb{R}$ uma função de Carathéodory. Suponhamos que existam $c>0, b \in L^{q}(\Omega), 1 \leq q \leq \infty$, e $r>0$ tais que

$$
|f(x, s)| \leq c|s|^{r}+b(x), \text { para todo } x \in \Omega \text { e } s \in \mathbb{R}
$$

Então $\widehat{f}: L^{q r}(\Omega) \rightarrow L^{q}(\Omega)$ é uma função contínua. Além disso a imagem de subconjuntos limitados é um conjunto limitado.

É impressionante saber que a condição suficiente do Teorema 1.2.2 é também necessária para um função de Carathéodory definir uma aplicação de Nemytskiǐ. Mais precisamente temos:

Teorema 1.2.3. Seja $\Omega$ um subconjunto aberto de $\mathbb{R}^{N}$ e seja $f: \Omega \times \mathbb{R} \rightarrow \mathbb{R}$ uma função de Carathéodory. Suponhamos que existam $p, q \in[1, \infty)$ tais que $\widehat{f}: L^{p}(\Omega) \rightarrow L^{q}(\Omega)$. Então existem $c>0$ e $b \in L^{q}(\Omega)$ tais que

$$
|f(x, s)| \leq c|s|^{p / q}+b(x), \text { para todo } x \in \Omega \text { e } s \in \mathbb{R}
$$

Para nossos objetivos precisamos, ainda, de condições que garantam a diferenciabilidade da aplicação de Nemytskiǐ. Para isso enunciamos o seguinte resultado sobre diferenciação.

Teorema 1.2.4. Seja $\Omega$ um subconjunto aberto de $\mathbb{R}^{N}$ e seja $f: \Omega \times \mathbb{R} \rightarrow \mathbb{R}$ uma função de Carathéodory. Suponhamos que existam $c>0, b \in L^{n}(\Omega), 1 \leq n \leq \infty$ e $m>0$ tais que

$$
\left|\frac{\partial f}{\partial s}(x, s)\right| \leq c|s|^{m}+b(x), \text { para todo } x \in \Omega \text { e } s \in \mathbb{R} .
$$

Então as aplicações de Nemytskǐ̌

$$
\widehat{f}: L^{p}(\Omega) \rightarrow L^{q}(\Omega) e \widehat{f}^{\prime}: L^{p}(\Omega) \rightarrow L^{n}(\Omega)
$$

onde $f^{\prime}=\frac{\partial f}{\partial s}, p=m n, q=\frac{m n}{m+1}$, estão bem definidas. Mais ainda $\widehat{f}$ é continuamente Fréchet 
diferenciável com $D \widehat{f}: L^{p}(\Omega) \rightarrow \mathscr{L}\left(L^{p}(\Omega), L^{q}(\Omega)\right)$ definida por

$$
\left.D \widehat{f}(u)[v]=\widehat{f}^{\prime}(u) v=f^{\prime}(\cdot, u(\cdot)) v(\cdot)\right) \text {, para todo } u, v \in L^{p}(\Omega) \text {. }
$$

\subsection{Medida de não-compacidade de Kuratowski}

Nosso objetivo nesta seção é definir uma medida que nos mostre, dado um subconjunto limitado de um espaço de dimensão infinita, o quão "não-compacto" é este conjunto. Uma referência para o tópico é [11]. Seja $X$ um espaço de Banach e denote por $\mathscr{B}$ o conjunto dos subconjuntos limitados em $X$.

No que segue dado um subconjunto $A$ de $X$, $\operatorname{diam}(A)$ denota o diâmetro do conjunto $A$. A bola aberta de centro em $a \in X$ e raio $r>0$ é denotada por $B(a, r)$.

Dado $A \in \mathscr{B}$ definimos

$$
\beta_{K}(A)=\inf \left\{d>0 \mid A \subset \bigcup_{j=1}^{n} K_{j} \operatorname{com} \operatorname{diam}\left(K_{j}\right) \leq d, j \in\{1, \ldots, n\}\right\} .
$$

A aplicação $\beta_{K}: \mathscr{B} \rightarrow[0, \infty)$ é chamada a medida de não-compacidade de Kuratowski. Dado $A \in \mathscr{B}$ podemos também definir

$$
\beta_{B}(A)=\inf \left\{r>0 \mid \text { existem } a_{j} \in X, j \in\{1, \ldots, n\}, \text { tais que } A \subset \bigcup_{j=1}^{n} B\left(a_{j}, r\right)\right\}
$$

A aplicação $\beta_{B}: \mathscr{B} \rightarrow[0, \infty)$ é chamada a medida de não-compacidade por bolas.

Com estas medidas definidas seguem os resultados que serão importantes no decorrer do trabalho.

Proposição 1.3.1. Suponha que X seja um espaço de Banach de dimensão infinita e seja $\mathscr{B}$ a família de limitados de $X$ e $\beta: \mathscr{B} \rightarrow[0, \infty)$ a medida de não-compacidade de Kuratowski ou a medida de não-compacidade por bolas. Então

(i) $\beta(A)=0$ se, e somente se, $\bar{A}$ é compacto.

(ii) $\beta$ é uma seminorma. 
(iii) Sejam $A_{1}, A_{2} \in \mathscr{B}$ tais que $A_{1} \subseteq A_{2}$. Então $\beta\left(A_{1}\right) \leq \beta\left(A_{2}\right)$ e $\beta\left(A_{1} \cup A_{2}\right)=$ $\max \left\{\beta\left(A_{1}\right), \beta\left(A_{2}\right)\right\}$.

(iv) $\beta(\operatorname{conv} A)=\beta(A)$, para todo $A \in \mathscr{B}$. Aqui conv $A$ denota a envoltória convexa de $A$.

(v) $\beta$ é contínua com respeito a distância de Hausdorff $\mathrm{d}_{H}$ dada por

$$
\mathrm{d}_{H}\left(A_{1}, A_{2}\right)=\max \left\{\sup _{x \in A_{1}} \inf _{y \in A_{2}}|x-y|, \sup _{x \in A_{2}} \inf _{y \in A_{1}}|x-y|\right\}, A_{1}, A_{2} \in \mathscr{B} .
$$

Em particular, $\beta(A)=\beta(\bar{A})$.

\subsection{Operadores $m$-dissipativos}

Nesta seção definiremos os chamados operadores dissipativos e $m$-dissipativos. Tais operadores são usualmente encontrados em exemplos e possuem boas propriedades para nossos objetivos. As demonstrações dos resultados apresentados podem ser encontradas em [6]. No que segue considere $X$ um espaço de Banach.

Definição 1.4.1. Um operador $A: D(A) \subset X \rightarrow X$ em $X$ é dito dissipativo se

$$
|u-\lambda A u| \geq|u|, \text { para todo } u \in D(A) \text { e todo } \lambda>0
$$

Definição 1.4.2. Um operador $A: D(A) \subset X \rightarrow X$ em $X$ é dito $m$-dissipativo se as seguintes condições estão satisfeitas:

(i) A é dissipativo;

(ii) para todo $\lambda>0$ e para todo $v \in X$, existe $u \in D(A)$ tal que $u-\lambda A u=v$.

A proposição a seguir oferece uma condição necessária e suficiente para que um operador dissipativo seja também $m$-dissipativo.

Proposição 1.4.3. Seja $A: D(A) \subset X \rightarrow X$ um operador dissipativo em $X$. As seguintes propriedades são equivalentes:

(i) A é m-dissipativo em $X$. 
(ii) Existe um $\lambda_{0}>0$ tal que para todo $v \in X$, a equação $u-\lambda_{0} A u=v$ possui uma solução $u \in D(A)$.

Agora, vamos restringir ao caso em que $X$ é um espaço de Hilbert e analisar o conceito de dissipatividade e $m$-dissipatividade. Seja $X$ um espaço de Hilbert. O produto interno definido em $X$ será denotado por $\langle\cdot, \cdot\rangle$. No que segue dado $A: D(A) \subset X \rightarrow X$ um operador linear densamente definido, o seu operador adjunto será denotado por $A^{*}$.

Apresentamos um critério de dissipatividade para operadores definidos em espaços de Hilbert:

Proposição 1.4.4. Um operador $A: D(A) \subset X \rightarrow X$ é dissipativo em $X$ se, e somente se, $\langle A u, u\rangle \leq 0$, para todo $u \in D(A)$.

Uma consequência importante é o seguinte

Corolário 1.4.5. Seja $A: D(A) \subset X \rightarrow X$ um operador m-dissipativo em $X$. Então $D(A)$ é denso em $X$.

Os resultados a seguir relacionam operadores dissipativos e $m$-dissipativos com o operador adjunto e operadores auto-adjuntos.

Teorema 1.4.6. Seja $A: D(A) \subset X \rightarrow X$ um operador linear densamente definido e dissipativo em X. Então A é m-dissipativo se, e somente se, A é um operador fechado e $A^{*}$ é um operador dissipativo.

Corolário 1.4.7. Seja $A: D(A) \subset X \rightarrow X$ um operador auto-adjunto em $X$ tal que $\langle A u, u\rangle \leq 0$, para todo $u \in D(A)$. Então A é m-dissipativo.

Proposição 1.4.8. Seja $A: D(A) \subset X \rightarrow X$ um operador simétrico e densamente definido tal que $\langle A u, u\rangle \leq 0$ para todo $u \in D(A)$ em $X$. Então A é m-dissipativo se, e somente se, A é auto-adjunto.

\subsection{Operadores setoriais}

Operadores setoriais têm uma relevância muito grande no estudo dos semigrupos lineares, pois estes são geradores dos conhecidos semigrupos analíticos que possuem inúmeras aplicações no estudo das equações diferenciais parciais parabólicas. 
Nesta seção faremos uma breve exposição dos operadores setorias e apresentamos as notações e resultados que serão utilizados no texto. Em [7], [8] e [15] encontramos mais detalhes sobre o assunto.

Iniciamos recordando algumas definições e resultados da teoria geral de semigrupos.

Nesta seção $X$ denota um espaço de Banach e denotamos por $\mathscr{L}(X)$ o conjunto dos operadores lineares limitados de $X$ em $X$.

Definição 1.5.1. Um semigrupo de operadores linerares ou um $C^{0}$-semigrupo é uma família de operadores lineares $\{T(t) \mid t \geq 0\}$ em $\mathscr{L}(X)$ que satisfaz as seguintes propriedades:

(i) $T(0) x=x$, para todo $x \in X$.

(ii) $T(t+s) x=T(t) T(s) x$, para todo $s, t \geq 0$ e $x \in X$.

(iii) $T(t) x \rightarrow x$, quando $t \rightarrow 0^{+}$, para todo $x \in X$.

Definição 1.5.2. Um semigrupo uniformemente contínuo de operadores é uma família de operadores lineares $\{T(t) \mid t \geq 0\}$ em $\mathscr{L}(X)$ que satisfaz as seguintes propriedades:

(i) $T(0) x=x$, para todo $x \in X$.

(ii) $T(t+s) x=T(t) T(s) x$, para todo $s, t \geq 0$ e $x \in X$.

(iii) $\|T(t)-I\| \rightarrow 0$, quando $t \rightarrow 0^{+}$, para todo $x \in X$.

É claro que todo semigrupo uniformemente contínuo de operadores é um $C^{0}$-semigrupo.

Teorema 1.5.3. Seja $\{T(t) \mid t \geq 0\}$ um $C^{0}$-semigrupo em $X$. Então existem constantes $\omega \geq 0 e$ $M \geq 1$ tais que

$$
\|T(t)\| \leq M e^{\omega t} \text {, para todo } t \in[0, \infty) .
$$

O resultado acima nos mostra que um $C^{0}$-semigrupo é uniformemente limitado em intervalos limitados da reta.

Corolário 1.5.4. Se $\{T(t) \mid t \geq 0\}$ é um $C^{0}$-semigrupo em $X$, então $[0, \infty) \times X \ni(t, x) \mapsto T(t) x \in$ $X$ é uma aplicação contínua. 
Seja $\{T(t) \mid t \geq 0\}$ um $C^{0}$-semigrupo em $X$ e considere o conjunto

$$
D=\left\{x \in X \mid \lim _{t \rightarrow 0^{+}} \frac{T(t) x-x}{t} \text { existe }\right\}
$$

Definimos o operador linear $A: D(A) \subset X \rightarrow X$, onde $D(A)=D$ e

$$
A x=\lim _{t \rightarrow 0^{+}} \frac{T(t) x-x}{t}, \text { para } x \in D(A) .
$$

O operador $A$ definido acima é chamado gerador infinitesimal do semigrupo $\{T(t)) \mid t \geq 0\}$ em $X$.

Teorema 1.5.5. Sejam $\{T(t) \mid t \geq 0\}$ um $C^{0}$-semigrupo em $X$ e $A: D(A) \subset X \rightarrow X$ seu gerador infinitesimal. As seguintes propriedades são satisfeitas:

1. $\lim _{h \rightarrow 0^{+}} \frac{1}{h} \int_{t}^{t+h} T(\tau) d \tau=T(t) x$, para todo $x \in X, t \in[0, \infty)$.

2. $\int_{0}^{t} T(\tau) x d \tau \in D(A)$ e $A\left(\int_{0}^{t} T(\tau) x d \tau\right)=T(t) x-x$, para todo $x \in X, t \in[0, \infty)$.

3. $T(t) x \in D(A)$, se $x \in D(A)$ e $t \in[0, \infty)$. Além disso, para $x \in D(A)$, a função $[0, \infty) \ni t \mapsto$ $T(t) x \in X$ é diferenciável e

$$
\frac{d}{d t} T(t) x=A T(t) x=T(t) A x
$$

4. $T(t) x-T(s) x=\int_{s}^{t} T(\tau) A x d \tau=\int_{s}^{t} A T(\tau) x d \tau$, para $x \in D(A)$ e $t, s \in[0, \infty)$, com $s \leq t$.

Corolário 1.5.6. Se $A: D(A) \subset X \rightarrow X$ denota o gerador infinitesimal de um $C^{0}$-semigrupo $\{T(t) \mid t \geq 0\}$, então A é um operador linear fechado e $\overline{D(A)}=X$.

Passamos à definição de operadores setorias e listaremos alguns resultados importantes. Dados $\alpha \in \mathbb{R}$ e $\phi \in(0, \pi / 2)$, definimos o setor $S_{\alpha, \phi}$ do plano complexo por

$$
S_{\alpha, \phi}:=\{\lambda \in \mathbb{C}|\phi \leq| \arg (\lambda-\alpha) \mid \leq \pi, \lambda \neq \alpha\}
$$

Um operador linear fechado $A: D(A) \subset X \rightarrow X$ densamente definido em $X$ é dito um operador setorial em $X$ se existirem constantes $\alpha \in \mathbb{R}, \phi \in(0, \pi / 2)$ e $M>0$ tais que 
(i) $\rho(A)$ contém o setor $S_{\alpha, \phi}$,

(ii) para cada $\lambda \in S_{\alpha, \phi}$ vale a estimativa

$$
\left\|(\lambda-A)^{-1}\right\| \leq \frac{M}{|\lambda-\alpha|}
$$

Proposição 1.5.7. Seja $A: D(A) \subset X \rightarrow X$ um operador linear fechado e densamente definido em X. As seguintes afirmativas são equivalentes:

(a) A+ $A$ I é um operador setorial em $X$ para algum $\omega \in \mathbb{R}$.

(b) A+ $\mathrm{II}$ é um operador setorial em $X$ para todo $\omega \in \mathbb{R}$.

(c) Existem constantes $k, \omega \in \mathbb{R}$ tais que o conjunto $\rho\left(A_{\omega}\right)$ contém o semiplano $\{\lambda \in \mathbb{C} \mid$ $\operatorname{re} \lambda \leq k\} e$

$$
\left\|\lambda\left(\lambda I-A_{\omega}\right)^{-1}\right\| \leq M, \text { para todo re } \lambda \leq k
$$

Aqui, $A_{\omega}:=A+\omega I$.

Suponhamos que $\{T(t) \mid t \geq 0\}$ seja um $C^{0}$-semigrupo de operadores lineares em $X$ e que existem um setor do plano complexo

$$
\Delta_{\phi}=\{z \in \mathbb{C}|| \arg z \mid<\phi\}, \operatorname{com} 0<\phi \leq \pi / 2,
$$

e uma família $\left\{T(z) \mid z \in \Delta_{\phi}\right\}$ de operadores em $\mathscr{L}(X)$ que coincide com $T(t)$ para $z=t \in[0, \infty)$ e tal que

(i) a aplicação $z \mapsto T(z)$ é analítica em $\Delta_{\phi} \backslash\{0\}$,

(ii) $T\left(z_{1}+z_{2}\right)=T\left(z_{1}\right) T\left(z_{2}\right)$, se $z_{1}, z_{2} \in \Delta_{\phi}$,

(iii) para todo $x \in X, \lim _{\substack{z \rightarrow 0 \\ z \in \Delta_{\phi}}} T(z) x=x$.

O $C^{0}$-semigrupo $\{T(t) \mid t \geq 0\}$ é chamado semigrupo analítico (fortemente contínuo).

Lema 1.5.8. Seja $\{T(t) \mid t \geq 0\}$ um semigrupo analítico em $\Delta_{\phi}$, com $0<\phi \leq \pi / 2$. Existem $M \geq 1$ e $\omega \geq 0$ tais que $\|T(z)\| \leq M e^{\omega \text { re } z}, z \in \Delta_{\phi}$. 
O teorema a seguir caracteriza os geradores de semigrupos analíticos e uma prova detalhada pode ser encontrada em [8].

Teorema 1.5.9. Seja $A: D(A) \subset X \rightarrow X$ um operador linear fechado e densamente definido. As seguintes afirmativas são equivalentes:

(i) A é o gerador infinitesimal de um semigrupo analítico.

(ii) -A é um operador setorial em $X$.

Lema 1.5.10. Seja $0<\phi \leq \pi / 2$. Seja $\{T(t) \mid t \geq 0\}$ um semigrupo analítico no setor $\Delta_{\phi}=$ $\{z \in \mathbb{C}|| \arg z \mid<\phi\}$, tal que para constantes $C \geq 1$ e a $\in \mathbb{R}$,

$$
\|T(z)\| \leq C e^{-a \mathrm{re} z}, z \in \Delta_{\phi}
$$

e suponha que $A: D(A) \subset X \rightarrow X$ seja seu gerador infinitesimal. As seguintes propriedades estáo satisfeitas:

(1) $T(z)=\frac{1}{2 \pi i} \int_{\Gamma} e^{\lambda z}(\lambda-A)^{-1} d \lambda$, para $z \in \Delta_{\Phi} \backslash\{0\}$, onde $\Gamma$ é a curva consistente dos seguintes segmentos orientados conforme a parametrização:

$$
\begin{aligned}
& \Gamma_{1}=\left\{-a-r e^{-i(\pi / 2+\phi)} \mid-\infty<r \leq 1\right\} ; \\
& \Gamma_{2}=\left\{-a+e^{i \psi} \mid-\pi / 2-\phi \leq \psi \leq \pi / 2+\phi\right\} ; \\
& \Gamma_{3}=\left\{-a+r e^{i(\pi / 2+\phi)} \mid 1 \leq r<\infty\right\} .
\end{aligned}
$$

(2) Para todo $0<\varepsilon<\phi$ e $x \in X$, temos

$$
T(z) x \rightarrow x \text {, se } z \rightarrow 0 \text { e } z \in \Delta_{\phi-\varepsilon} .
$$

(3) Se $t>0$, então $T(t) x \in D(A)$ para todo $x \in X$ e $\frac{d}{d t} T(t) x=A T(t) x$, para todo $x \in X$.

Nosso intuito agora é definirmos as potências fracionárias que são elementos importantes no estudo das equações parabólicas e suas soluções.

Definição 1.5.11. Um operador $A: D(A) \subset X \rightarrow X$ será dito operador positivo se as seguintes condições forem satisfeitas: 
(i) A é um operador fechado e densamente definido,

(ii) $(-\infty, 0] \subset \rho(A) e$

(iii) existe um $N \geq 1$ tal que

$$
\left\|(s-A)^{-1}\right\| \leq \frac{N}{1+|s|}, \text { para todo } s \leq 0
$$

O operador $A$ da definição acima também é dito operador positivo do tipo $N$, onde $N$ é como em (iii).

Lema 1.5.12. Suponha que $A: D(A) \subset X \rightarrow X$ seja um operador positivo do tipo $N$. Então o setor

$$
\Sigma_{N}=\left\{\lambda \in \mathbb{C} \mid \text { existe um } s \leq 0 \text { tal que }|\lambda-s| \leq \frac{1+|s|}{2 N}\right\}
$$

está contido em $\rho(A)$. Além disso

$$
\left\|(\lambda-A)^{-1}\right\| \leq \frac{2 N+1}{1+|\lambda|}, \text { para todo } \lambda \in \Sigma_{N}
$$

Nas condições do Lema 1.5 .12 pode ser mostrado que

$$
\left\{\lambda \in \mathbb{C}|| \arg \lambda \mid \geq \pi-\operatorname{arcsen} \frac{1}{2 N}\right\} \cup\left\{\lambda \in \mathbb{C}|| \lambda \mid \leq \frac{1}{2 N}\right\} \subset \Sigma_{N} .
$$

Lema 1.5.13. Seja $A: D(A) \subset X \rightarrow X$ um operador positivo do tipo $N$. Para cada $z \in \mathbb{C}$ tal que re $z \leq 0$, definimos

$$
B(z)=\frac{-1}{2 \pi i} \int_{\Gamma} \lambda^{z}(\lambda-A)^{-1} d \lambda
$$

onde $\Gamma$ é a curva em $\Sigma_{N} \backslash(-\infty, 0]$ que consiste dos três segmentos

$$
\begin{aligned}
& \Gamma_{1}=\left\{-s e^{-i \theta} \mid s \in(-\infty,-1 /(4 N))\right\}, \\
& \Gamma_{2}=\left\{(1 /(4 N)) e^{i \psi}|| \psi \mid \leq \theta\right\}, \\
& \Gamma_{3}=\left\{s e^{i \theta} \mid s \in[1 /(4 N), \infty)\right\},
\end{aligned}
$$

onde $\theta \in[\pi-\operatorname{arcsen}(1 /(2 N)), \pi)$, orientada pela parametrização. Então para $z$ fixado $B(z)$ está bem definida, isto é, não depende da escolha de $\theta$. Além disso, $B(z) \in \mathscr{L}(X)$ para todo $z \in \mathbb{C}$ com re $z<0$ e também, a aplicação $z \mapsto B(z)$ é analítica em $\{z \in \mathbb{C} \mid$ re $z<0\}$. 
Note que, o Teorema de Cauchy implica que podemos modificar o comportamento de $\Gamma$ em torno do $0 \in \mathbb{C}$. E, com o Lema 1.5 .13 , podemos definir as potências fracionárias de um operador positivo, se re $z<0$.

Definição 1.5.14. Seja $A: D(A) \subset X \rightarrow X$ um operador positivo do tipo $N$. Para $z \in \Pi_{0}=\{z \in$ $\mathbb{C} \mid$ re $z<0\}$, definimos

$$
A^{z}:=\frac{-1}{2 \pi i} \int_{\Gamma} \lambda^{z}(\lambda-A)^{-1} d \lambda \in \mathscr{L}(X)
$$

onde $\Gamma$ é dada como no Lema $1.5 .13 \operatorname{com} \theta \in[\pi-\operatorname{arcsen}(1 /(2 N)), \pi)$.

No que segue utilizaremos a notação $A^{0}=I$, onde $I$ denota a a aplicação identidade em $X$.

Proposição 1.5.15. Seja $A: D(A) \subset X \rightarrow X$ um operador positivo. Então

(i) $A^{z_{1}+z_{2}}=A^{z_{1}} \circ A^{z_{2}}$, para quaisquer $z_{1}, z_{2} \in \Pi_{0} \cup\{0\}$.

(ii) Para $n \in \mathbb{N}$, temos $A^{-n}=\left(A^{-1}\right)^{n}$, onde $A^{-1}$ denota a inversa de $A$.

(iii) $A^{z}$ é injetor, para cada $z \in \mathbb{C}$ com re $z<0$.

Segue da Proposição 1.5 .15 que podemos definir as potências fracionárias de um operador positivo quando re $z>0$.

Definição 1.5.16. Seja $A: D(A) \subset X \rightarrow X$ um operador positivo. Definimos

$$
A^{z}=\left(A^{-z}\right)^{-1}: R\left(A^{-z}\right) \subset X \rightarrow X
$$

para $z \in \mathbb{C}$ com re $z>0$.

Podemos também definir a potência fracionária de um operador positivo quando re $z=0$, porém este caso não será importante nos nossos estudos e portanto não será retratado nesta seção. Abaixo, enumeramos fatos importantes sobre as potências fracionárias de um operador positivo.

Teorema 1.5.17. Seja $A: D(A) \subset X \rightarrow X$ um operador positivo. As seguintes propriedades são satisfeitas: 
(1) $A^{z}$ é um operador fechado em $X$, para todo $z \in \mathbb{C}$, com re $z>0$,

(2) se $z_{1}, z_{2} \in \mathbb{C}$, com re $z_{1}>$ re $z_{2}>0$, então $D\left(A^{z_{1}}\right) \subset D\left(A^{z_{2}}\right) \subset X$,

(3) para cada $z \in \mathbb{C}$, com re $z>0$, temos $\overline{D\left(A^{z}\right)}=X$.

Dizemos que um operador $A: D(A) \subset X \rightarrow X$ é um operador setorial positivo se $A$ é setorial em $X$ e re $\sigma(A)>0$.

Lema 1.5.18. Suponha que $-A: D(A) \subset X \rightarrow X$ seja o gerador infinitesimal de um semigrupo $\{T(t) \mid t \geq 0\}$ e que a $>0$ e $M \geq 1$ sejam constantes positivas tais que

$$
\|T(t)\| \leq M e^{-a t}, \text { para todo } t \geq 0
$$

Então A é um operador positivo.

Observamos que em particular, segue do Lema 1.5 .18 que se $A$ é um operador setorial positivo, então $A$ é um operador positivo.

Teorema 1.5.19. Seja $-A: D(A) \subset X \rightarrow X$ o gerador infinitesimal de um semigrupo $\{T(t) \mid$ $t \geq 0\}$ e suponha que existam constantes $a>0$ e $M \geq 1$ tais que

$$
\|T(t)\| \leq M e^{-a t}, \text { para todo } t \geq 0
$$

Então, se $z \in \mathbb{C}$ com re $z>0$, temos

$$
A^{-z} x=\frac{1}{\Gamma(z)} \int_{0}^{\infty} t^{z-1} T(t) x d t, x \in X
$$

Vamos agora restringir o estudo às potências fracionárias reais de operadores positivos. Seja $A: D(A) \subset X \rightarrow X$ um operador positivo. Dado $\alpha \geq 0, X^{\alpha}$ denota o espaço $D\left(A^{\alpha}\right)=R\left(A^{-\alpha}\right)$. Em $X^{\alpha}$ consideraremos a com a norma

$$
|u|_{X^{\alpha}}=\left|A^{\alpha} u\right|, u \in D\left(A^{\alpha}\right) .
$$

A família $\left\{X^{\alpha}\right\}_{\alpha \geq 0}$ é uma família de espaços de Banach. 
Lema 1.5.20. Seja $0 \leq \alpha \leq \beta$, então $X^{\beta}$ é um subespaço denso de $X^{\alpha}$ e a inclusão $X^{\beta} \rightarrow X^{\alpha}$ é contínua, mais precisamente, existe uma constante $C \geq 0$ tal que

$$
|u|_{X^{\alpha}} \leq C|u|_{X^{\beta}} \text {, para todo } u \in X^{\beta} \text {. }
$$

Teorema 1.5.21. Seja $A: D(A) \subset X \rightarrow X$ um operador positivo. Se $0 \leq \alpha<1$, então existe uma constante $c>0$ tal que para todo $x \in X^{1}$, temos

$$
|x|_{X^{\alpha}} \leq c|x|_{X}^{1-\alpha}|x|_{1}^{\alpha}
$$

Teorema 1.5.22. Sejam A um operador setorial positivo e $\{T(t) \mid t \geq 0\}$ o semigrupo analítico gerado por $-A$. As seguintes propriedades são satisfeitas:

(i) $T(t): X \rightarrow D\left(A^{\alpha}\right)$, para todo $\alpha \geq 0$ e $t>0$.

(ii) $T(t) A^{\alpha} u=A^{\alpha} T(t) u$, para todo $\alpha \geq 0, u \in D\left(A^{\alpha}\right)$ e $t \geq 0$.

(iii) $A^{\alpha} T(t) \in \mathscr{L}(X)$. Além disso existe um a $>0$ e para cada $\alpha \geq 0$ existe uma constante positiva $C_{\alpha}$ tal que

$$
\left\|A^{\alpha} T(t)\right\| \leq C_{\alpha} t^{-\alpha} e^{-a t}, \text { para todo } t>0
$$

(iv) Se $0<\alpha \leq 1$, então

$$
\|(T(t)-I) u\| \leq \frac{1}{\alpha} C_{1-\alpha} t^{\alpha}\left|A^{\alpha} u\right|, \text { para todo } u \in D\left(A^{\alpha}\right) \text { e } t>0
$$

onde $C_{1-\alpha}$ é a constante positiva de (iii).

Proposição 1.5.23. Sejam $\alpha, \gamma \geq 0$ e $\theta \in[0,1]$. Escreva $\beta=(1-\theta) \alpha+\theta \gamma$. Então existe constante $C \geq 0$ tal que:

$$
\left|A^{\beta} u\right| \leq C\left|A^{\alpha} u\right|^{1-\theta}\left|A^{\gamma} u\right|^{\theta} \text {, para cada } u \in X
$$

O seguinte resultado é encontrado em [13] e [15].

Lema 1.5.24. Sejam $X, Y$ espaços de Banach, $A: D(A) \subset X \rightarrow X$ um operador setorial com $\operatorname{re} \sigma(A)>0$ e $B: D(B) \subset X \rightarrow Y$ uma transformação linear. Suponhamos que $D(B) \supset D(A) e$ 
que existam $\alpha \in[0,1)$ e c $\geq 0$ tais que

$$
|B x|_{Y} \leq c|A x|^{\alpha}|x|^{1-\alpha}, \text { para todo } x \in D(A)
$$

Então para todo $\beta \in(\alpha, 1]$, B possui uma única extenso a um transformação linear contínua de $X^{\beta}$ em $Y$, isto é, $B A^{-\beta}$ é transformação linear contínua.

Finalizamos esta seção enunciando alguns resultados sobre operadores setoriais para o caso em que $X$ for um espaço de Hilbert. A seguir apresentamos uma condição suficiente para que um operador seja setorial para espaços de Hilbert.

Proposição 1.5.25. Suponha que $(X,\langle\cdot, \cdot\rangle)$ seja um espaço de Hilbert. Seja $A: D(A) \subset X \rightarrow X$ um operador linar auto-adjunto, densamente definido e suponha que exista uma constante $a \in \mathbb{R}$ tal que

$$
\langle A x, x\rangle \geq a\langle x, x\rangle \text {, para todo } x \in D(A) .
$$

Então A é setorial.

Corolário 1.5.26. Seja $(X,\langle\cdot, \cdot\rangle)$ seja um espaço de Hilbert. Se $A: D(A) \subset X \rightarrow X$ é autoadjunto, densamente definido e re $\sigma(A)>0$, então A é setorial.

No caso particular em que $X$ é um espaço de Hilbert, se $A$ for auto-adjunto e definido positivo, então $A$ será setorial positivo, como enunciado no Corolário 1.5.26.

Finalmente, em vista da teoria espectral para operadores auto-adjuntos feita na Seção 1.1 se $A$ for auto-adjunto, setorial positivo em $X$ espaço de Hilbert, podemos escrever:

$$
A^{-\alpha}=\int_{\mathbb{R}} \lambda^{-\alpha} d E_{\lambda}
$$

onde $\left\{E_{\lambda}\right\}_{\lambda \in \mathbb{R}} \subset \mathscr{L}(X)$ representa a família espectral gerada pelo operador $A$ em $X$. Portanto, a partir de uma aplicação da Desigualdade de Hölder em (1.5.5), obtemos a

Proposição 1.5.27. Sejam $\alpha, \gamma \leq 0$, tais que $\alpha \leq \gamma$, e $\theta \in[0,1]$. Escreva $\beta=(1-\theta) \alpha+\theta \gamma$. Então:

$$
\left|A^{\beta} u\right| \leq\left|A^{\alpha} u\right|^{1-\theta}\left|A^{\gamma} u\right|^{\theta} \text {, para todo } u \in X
$$




\subsection{Semifluxos e atratores globais}

Neste trabalho estamos interessados na existência de atratores. Os atratores são importantes objetos no estudo da dinâmica assintótica dos sistemas dinâmicos. Nesta seção vamos expor os principais elementos para a definição dos atratores e listar suas propriedades importantes. Também apresentaremos condições necessárias e suficientes para que um sistema dinâmico possua atrator.

As referências [5], [7], [14] e [17] apresentam a teoria de atrator global para sistemas dinâmicos não lineares.

Para esta seção, a referência [7] foi utilizada para o estudo dos atratores e o conceito de semifluxo local é como apresentado em [22].

Sejam $X$ um espaço métrico e $D$ um aberto de $[0, \infty) \times X$. Uma aplicação $\pi: D \rightarrow X$ é um semifluxo local em $X$ se

(a) para cada $u \in X$, existe um $\omega_{u}=\omega(\pi, u) \in(0, \infty]$ tal que $(t, u) \in D$ se, e somente se, $t \in\left[0, \omega_{u}\right)$

(b) $\pi(0, u)=u$, para todo $u \in X$;

(c) sempre que $(t, u) \in D$ e $(s, u \pi t) \in D$, temos $(t+s, u) \in D$ e $\pi(t+s, u)=\pi(s, \pi(t, u))$.

Escrevemos $\pi(t, u)=u \pi t$, para $(t, u) \in D$.

Se um semifluxo é tal que $\omega_{u}=+\infty$ para todo $u \in X$, então dizemos que este é um semifluxo global.

Dado um intervalo $I \subset \mathbb{R}$, uma aplicação $\sigma: I \rightarrow \mathbb{R}$ é chamada uma solução de $\pi$ se sempre que $t \in I$ e $s \in[0, \infty)$ são tais que $t+s \in I$, então $\sigma(t) \pi s$ é bem definido e $\sigma(t) \pi s=\sigma(t+s)$. $\operatorname{Se} I=\mathbb{R}$ então $\sigma$ é chamada solução global de $\pi$.

Um subconjunto $A$ de $X$ é chamado $\pi$-invariante se para todo $u \in A$ existe uma solução global $\sigma$ tal que $\sigma(\mathbb{R}) \subset A$ e $\sigma(0)=u$.

Um ponto $u \in X$ é chamado equilíbrio $(d e \pi)$ se $u \pi t=u$, para todo $t \in\left[0, \omega_{u}\right)$. 
Dados um semifluxo local $\pi$ em $X$ e um subconjunto $N$ de $X$, dizemos que $\pi$ não explode em $N$ se sempre que $u \in X$ e $u \pi\left[0, \omega_{u}\right) \subset N$, implicar $\omega_{u}=+\infty$.

No restante desta seção $\pi$ denota um semifluxo global definido num espaço de Banach $X$.

A seguinte definição é apresentada em [7].

Definição 1.6.1. Dizemos que um subconjunto $B \subset X$ é ( $\pi$-)eventualmente limitado se existe $u m t_{B} \in[0, \infty)$ tal que o conjunto $\left\{u \pi t \mid u \in B, t \in\left[t_{B}, \infty\right)\right\}$ é limitado.

Pode ser encontrado na literatura o conceito de semifluxo (semigrupo) eventualmente limitado. Neste caso, dizemos $\pi$ é eventualmente limitado se todo limitado de $X$ é eventualmente limitado conforme a Definição 1.6.1

Seja $B \subset X$. A órbita positiva de $B$ por $\pi$ é definida como o conjunto

$$
\gamma^{+}(B):=\bigcup_{t \in[0, \infty)} B \pi t=\bigcup_{t \in[0, \infty)}\{u \pi t \mid u \in B\}
$$

O conjunto

$$
\gamma_{t}^{+}(B):=\bigcup_{s \in[0, \infty)} B \pi(s+t)=\bigcup_{s \in[t, \infty)} B \pi s
$$

é chamadoa órbita de $B \pi t$. O conjunto $\omega$-limite de $B$ é definido como

$$
\omega(B)=\bigcap_{t \in[0, \infty)} \overline{\gamma_{t}^{+}(B)}
$$

Proposição 1.6.2. Seja $B \subset X$. Então

(i) $\omega(B)$ é fechado.

(ii) Seja $v \in X$. Então $v \in \omega(B)$ se, e somente se, existem sequências $\left(t_{n}\right)_{n}$ em $[0, \infty) e\left(u_{n}\right)_{n}$ em B tais que $t_{n} \rightarrow \infty$ e $u_{n} \pi t_{n} \rightarrow v$ quando $n \rightarrow \infty$.

Precisamos da noção de atração sobre o semifluxo $\pi$ de modo a definirmos o conceito do atrator. Mas primeiro vamos definir a semidistância de Hausdorff entre dois conjuntos. Sejam $A$ e $B$ subconjuntos de $X$. Definimos a semidistância de Hausdorff entre $A$ e $B$, denotada por 
$\operatorname{dist}_{H}(A, B)$, por

$$
\operatorname{dist}_{H}(A, B)=\sup _{u \in A} \inf _{v \in B}|u-v|
$$

Sejam $A$ e $B$ subconjuntos de $X$. Dizemos que $A$ atrai $B$ pela ação de $\pi$ se

$$
\lim _{t \rightarrow \infty} \operatorname{dist}_{H}(B \pi t, A)=0 .
$$

Proposição 1.6.3. Seja $\pi$ um semifluxo global em $X$. Então $A \subset X$ é $\pi$-invariante se, e somente se, $A \pi t=A$, para todo $t \in[0, \infty)$.

Um subconjunto $\mathscr{A}$ de $X$ é um atrator global para $\pi$, se $\mathscr{A}$ é um conjunto compacto, $\pi$ invariante e que atrai subconjuntos limitados de $X$ sob a ação de $\pi$.

Definição 1.6.4. O semifluxo global $\pi$ é chamado assintoticamente suave se para cada subconjunto $B$ de $X$ não-vazio, fechado e limitado tal que $B \pi t \subseteq B$ para todo $t \in[0, \infty)$, existir um conjunto não-vazio e compacto $K \subset B$ tal que $K$ atrai $B$ sob ação de $\pi$.

Definição 1.6.5. O semifluxo global $\pi$ é chamado assintoticamente compacto se para cada subconjunto B de X não-vazio e eventualmente limitado tal que para cada sequência $\left(t_{n}\right)_{n}$ em $[0, \infty)$ e cada sequência $\left(u_{n}\right)_{n}$ em $B$ com $t_{n} \rightarrow \infty$, a sequência $\left(u_{n} \pi t_{n}\right)_{n}$ possui uma subsequência convergente.

Os conceitos acima são equivalentes:

Proposição 1.6.6. Um semifluxo global é assintoticamente suave se, e somente se, é assintoticamente compacto.

Teorema 1.6.7. Seja $\pi$ um semifluxo global definido num espaço de Banach X. As seguintes afirmativas são equivalentes:

(i) $\pi$ é assintoticamente compacto, existe um subconjunto limitado B de X tal que para cada $u \in X$ existe um $t_{u} \geq 0$ com $u \pi t_{u} \in B$ e todo conjunto limitado em $X$ é $\pi$-eventualmente limitado.

(ii) $\pi$ possui um atrator global $\mathscr{A}$. 
A seguir definiremos o que é conhecido por funcional de Lyapunov. Semifluxos que possuem um funcional de Lyapunov são chamados de semifluxos gradiente e possuem boas propriedades que nos levam a um teorema de existência de atrator global mais direto do que o Teorema 1.6 .7$.

Um funcional $\mathscr{L}: X \rightarrow \mathbb{R}$ contínuo em $X$ e limitado inferiormente é chamado funcional de Lyapunov para $\pi$ se

1. para cada $u \in X$ a aplicação $(0, \infty) \ni t \mapsto \mathscr{L}(u \pi t) \in \mathbb{R}$ é não-crescente,

2. se $u \in X$ é tal que existe um $k_{u} \in \mathbb{R}$ com

$$
\mathscr{L}(u \pi t)=k_{u}, \text { para todo } t \geq 0,
$$

então $u$ é um ponto de equilíbrio de $\pi$.

Podemos agora, apresentar o teorema que será utilizado para demonstrar a existência de atrator global no nosso trabalho.

Teorema 1.6.8. Seja $\pi$ um semifluxo global definido num espaço de Banach X. Suponha que:

(a) $\pi$ é assintoticamente compacto,

(b) todo subconjunto limitado em X é eventualmente limitado,

(c) o conjunto dos pontos de equilíbrio de $\pi$ é limitado $e$

(d) existe um funcional de Lyapunov para o semifluxo $\pi$.

Então existe um atrator global para $\pi$.

\subsection{Equações diferenciais parabólicas}

Nesta última seção apresentamos resultados utilizados sobre as equações diferenciais parabólicas. Seguimos a notação e resultados apresentados em [15] e [18]. 
Suponha $0 \leq \alpha<1$ e seja $U$ um conjunto aberto em $X^{\alpha}$. Seja $g: U \rightarrow X$ uma função localmente Lipschitziana. Considere a equação:

$$
\frac{d u}{d t}+A u=g(u)
$$

Seja $u_{0} \in U$ e $t_{0} \geq 0$. Uma solução de (1.7.1) em $\left(t_{0}, t_{1}\right)$ tal que em $t_{0}$ vale $u_{0}$ é uma função contínua $u:\left[t_{0}, t_{1}\right) \rightarrow X$ tal que

1. $u\left(t_{0}\right)=u_{0}$;

2. $u(t) \in U$ para $t \in\left(t_{0}, t_{1}\right)$;

3. $u$ é diferenciável em $\left(t_{0}, t_{1}\right)$;

4. $u(t) \in D(A)$ para $t \in\left(t_{0}, t_{1}\right)$;

5. $\left[t_{0}, t_{1}\right) \ni t \mapsto g(u(t)) \in X$ é contínua;

6. a equação (1.7.1) está satisfeita para todo $t \in\left(t_{0}, t_{1}\right)$.

A definição de solução é como em [18].

A seguir apresentamos um teorema de existência e unicidade de soluções maximais para a equação 1.7.1 com condição inicial $u\left(t_{0}\right)=u_{0} \in U$ cuja demonstração é adaptada das demonstrações de [15].

Teorema 1.7.1. Seja A um operador setorial em $X$ e seja $g: U \rightarrow X$ uma função localmente Lipschitziana, onde $U$ é um conjunto aberto em $X^{\alpha}$, para algum $0 \leq \alpha<1$. Então para todo $t_{0} \geq 0$ e para todo $u_{0} \in U$, existe um intervaldo maximal $\left[t_{0}, \omega_{u_{0}}\right)$, onde $\omega_{u_{0}} \in(0, \infty]$ e uma única solução $t \mapsto u\left(t, u_{0}\right)$ de

$$
\frac{d u}{d t}+A u=g(u)
$$

tal que $u\left(t_{0}\right)=u_{0}$ definida em $\left[t_{0}, \omega_{u_{0}}\right)$.

No restante do texto se $A: D(A) \subset X \rightarrow X$ é um operador setorial em $X$, o semigrupo analítico gerado por $-A$ será denotado por $\left\{e^{-A t} \mid t \geq 0\right\}$.

Para demonstrar a existência de solução no Teorema 1.7.1 utilizamos o seguinte lema: 
Lema 1.7.2. Seja u uma solução do problema 1.7 .1 em $\left(t_{0}, t_{1}\right)$ com valor inicial $u\left(t_{0}\right)=u_{0}$. Então

$$
u(t)=e^{-A\left(t-t_{0}\right)} u_{0}+\int_{t_{0}}^{t} e^{-A(t-s)} g(u(s)) d s, \text { para } t \in\left[t_{0}, t_{1}\right) .
$$

Reciprocamente, se $u:\left[t_{0}, t_{1}\right) \rightarrow X^{\alpha} e\left[t_{0}, t_{1}\right) \ni t \mapsto g(u(t)) \in X$ são funções contínuas, e a equação integral (1.7.2) é satisfeita para $t \in\left(t_{0}, t_{1}\right)$, então u é uma solução da equação diferencial (1.7.1).

Para a demonstração do Lema 1.7.2, ver [15] e [18].

A fórmula 1.7.2 é conhecida como a Fórmula da Variação das Constantes.

No que segue vamos supor que $A$ é um operador setorial positivo, isto é, $A$ é setorial e re $\sigma(A)>0, g: X^{\alpha} \rightarrow X$ uma função Lipschitziana em limitados de $X^{\alpha}$, com $0 \leq \alpha<1$ e, também, que $t_{0}=0$. Para cada $u_{0} \in U$ e $t \in\left[0, \omega_{u_{0}}\right)$, defina $u_{0} \pi t:=u\left(t, u_{0}\right)$. Dessa forma $\pi$ é um semifluxo local em $U$.

Vamos assumir também que $g: U \rightarrow X$ seja uma função Lipschitziana em limitados, isto é, dado $B \subset U$ limitado existe uma constante $L_{B} \geq 0$ tal que

$$
|g(u)-g(v)|_{X} \leq L_{B}|u-v|, \text { para todo } u, v \in B
$$

A seguir enunciamos e demonstramos resultados gerais que serão utilizado nos próximos capítulos.

Proposição 1.7.3. Seja $B \subset U$ um subconjunto limitado tal que para cada $u \in B$, $u \pi t \in B$, para todo $t \in\left[0, \omega_{u}\right)$. Então $\omega_{u}=+\infty$.

Demonstração. Seja $u \in B$. Logo, $u \pi t \in B$, para todo $t \in\left[0, \omega_{u}\right)$. Suponhamos por absurdo que $\omega_{u}<\infty$. Sejam $0<\alpha<\beta<1$ e $s \in\left(0, \omega_{u}\right)$.

Afirmamos que o conjunto $\left\{|u \pi t|_{X^{\beta}} \mid t \in\left[s, \omega_{u}\right)\right\}$ é limitado. De fato, seja $t \in\left[s, \omega_{u}\right)$. Utili- 
zando a Fórmula da Variação das Constantes temos

$$
\begin{aligned}
|u \pi t|_{X^{\beta}} & \leq\left|e^{-A t} u\right|_{X^{\beta}}+\int_{0}^{t}\left|e^{-A(t-s)} g(u \pi s)\right|_{X^{\beta}} d s \\
& \leq\left|A^{\beta-\alpha} e^{-A t}\right|\left|A^{\alpha} u\right|+\int_{0}^{t}\left|A^{\beta} e^{-A(t-s)} g(u \pi s)\right|_{X} d s \\
& \leq \max \left\{C_{\beta-\alpha}, C_{\beta} L_{B}\right\}\left(t^{\beta-\alpha}|u|_{X^{\alpha}}+\int_{0}^{t}(t-s)^{-\beta} d s\right) \\
& \leq \max \left\{C_{\beta-\alpha}, C_{\beta} L_{B}, 1\right\}\left(t^{\beta-\alpha}|u|_{X^{\alpha}+t^{1-\beta}}\right) .
\end{aligned}
$$

Como $\left[s, \omega_{u}\right) \ni t \mapsto t^{\beta-\alpha}|u|_{X^{\alpha}}+t^{1-\beta}$ é limitada, a afirmativa está demonstrada.

Sejam $s \leq \tau<t<\omega_{u}$. A Fórmula da Variação das Constantes implica que

$$
u \pi t-u \pi \tau=\left(e^{-A(t-\tau)}-I\right) u \pi \tau+\int_{\tau}^{t} e^{-A(t-s)} g(u \pi s) d s
$$

Portanto,

$$
\begin{aligned}
|u \pi t-u \pi \tau|_{X^{\alpha}} & \leq \frac{1}{\beta-\alpha} C_{1-\beta+\alpha}\left\|A^{-\alpha}\right\|(t-\tau)^{\beta-\alpha}\left|u_{0} \pi \tau\right|_{X^{\beta}}+C_{\alpha} L_{B} \int_{\tau}^{t}(t-s)^{-\alpha} d s \\
& \leq \frac{1}{\beta-\alpha} C_{1-\beta+\alpha}\left\|A^{-\alpha}\right\|(t-\tau)^{\beta-\alpha}+C_{\alpha} L_{B}(t-\tau)^{1-\alpha} \\
& \leq \widetilde{C}(t-\tau)^{\beta-\alpha}
\end{aligned}
$$

onde $\widetilde{C}=\max \left\{\frac{1}{\beta-\alpha} C_{1-\beta+\alpha}\left\|A^{-\alpha}\right\|, C_{\alpha} L_{B}(t-\tau)^{1-\beta}\right\}$.

Como $\beta>\alpha$, o Critério de Cauchy implica que o limite, quando $t \rightarrow \omega_{u}^{-}$, existe. Seja $u_{1}$ o valor deste limite e defina $v(t)=u \pi t$, se $t \in\left[0, \omega_{u}\right)$ e $v\left(\omega_{u}\right)=u_{1}$. Segue que $v$ é uma solução de (1.7.1) com $v(0)=u$ definida em $\left[0, \omega_{u}\right]$. Porém isso contradiz a maximalidade de $\omega_{u}$. Portanto, $\omega_{u}=+\infty$ para todo $u \in B$.

O próximo resultado pode ser encontrado na Proposição 3.2.1 e Lema 3.2.1 em [7].

Proposição 1.7.4. Seja $B \subset X^{\alpha}$ um subconjunto limitado em $X^{\alpha}$. Então para todo $t \in\left(0, \omega_{B}\right)$, Bлt é limitado em $X^{\gamma}$, para cada $\alpha \leq \gamma \leq 1$.

Concluímos o capítulo com um resultado de regularidade que pode ser encontrado em [15]. Como apresentaremos a demonstração desse resultado, enunciamos um lema auxiliar. 
Lema 1.7.5 (Desigualdade de Gronwall Singular). Sejam $0 \leq \alpha<1,0 \leq \beta<1, a \geq 0, b \geq 0$ constantes e $T \in(0, \infty)$. Se $u:[0, T] \rightarrow \mathbb{R}$ é uma função integrável tal que

$$
0 \leq u(t) \leq a t^{-\alpha}+b \int_{0}^{t}(t-s)^{-\beta} u(s) d s, \text { para quase todo } t \in[0, T]
$$

então existe uma constante positiva $M$ tal que

$$
0 \leq u(t) \leq a M t^{-\alpha} \text {, para quase todo } t \in(0, T]
$$

Teorema 1.7.6. Assuma a notação apresentada acima. Seja u uma solução de (1.7.1) em $\left[t_{0}, t_{1}\right]$ e $\gamma \in(0,1)$. Então $\frac{d u}{d t} \in X^{\gamma}$ e existe uma constante $C>0$ tal que

$$
\left|\frac{d u}{d t}\right|_{X \gamma} \leq C\left(t-t_{0}\right)^{\alpha-\gamma-1}, \text { para } t_{0}<t \leq t_{1} .
$$

Demonstração. Seja $\beta>0$ tal que $\max \{\alpha, \gamma\}<\beta<1$.

Denotemos $f(t):=g(u(t))$, para $t \in\left[t_{0}, t_{1}\right]$. Como $\left[t_{0}, t_{1}\right]$ é compacto, sejam $B$ e $L$ constantes positivas tais que, para todo $t \in\left[t_{0}, t_{1}\right]$ e $h_{0} \geq 0$, com $t_{0} \leq t \leq t+h \leq t_{1}$ e $0 \leq h \leq h_{0}$,

$$
\begin{gathered}
|f(t)| \leq B \\
|f(t+h)-f(t)| \leq L|u(t+h)-u(t)| .
\end{gathered}
$$

Para $t_{0}<t<t+h \leq t_{1}$ temos:

$$
\begin{aligned}
u(t+h)-u(t) & =e^{-A\left(t+h-t_{0}\right)} u\left(t_{0}\right)+\int_{t_{0}}^{t+h} e^{-A(t+h-s)} f(s) d s \\
& -e^{-A\left(t-t_{0}\right)} u\left(t_{0}\right)-\int_{t_{0}}^{t} e^{-A(t-s)} f(s) d s \\
& =\left(e^{-A h}-I\right) e^{-A\left(t-t_{0}\right)} u\left(t_{0}\right)+\int_{t_{0}}^{t_{0}+h} e^{-A(t+h-s)} f(s) d s \\
& +\int_{t_{0}}^{t} e^{-A(t-s)}[f(s+h)-f(s)] d s .
\end{aligned}
$$


Desse modo,

$$
\begin{aligned}
|u(t+h)-u(t)|_{X^{\alpha}} & \leq\left|\left(e^{-A h}-I\right) e^{-A\left(t-t_{0}\right)} u\left(t_{0}\right)\right|_{X^{\alpha}}+\int_{t_{0}}^{t_{0}+h}\left|e^{-A(t+h-s)} f(s)\right|_{X^{\alpha}} d s \\
& +\int_{t_{0}}^{t}\left|e^{-A(t-s)} f(s+h)-f(s)\right|_{X^{\alpha}} d s \\
& \leq \beta^{-1} C_{1-\beta} h^{\beta}\left|A^{\beta} e^{-A\left(t-t_{0}\right)} A^{\alpha} u\left(t_{0}\right)\right|_{X}+\int_{t_{0}}^{t_{0}+h} C_{\alpha}(t+h-s)^{-\alpha} K B d s \\
& +\int_{t_{0}}^{t} C_{\alpha}(t-s)^{-\alpha} K L|u(s+h)-u(s)|_{X^{\alpha}} d s \\
& \leq \beta^{-1} C_{1-\beta} h^{\beta} C_{\beta}\left(t-t_{0}\right)^{-\beta} K\left|u\left(t_{0}\right)\right|_{X^{\alpha}}+C_{\alpha} K B h\left(t-t_{0}\right)^{-\alpha}+ \\
& +C_{\alpha} K L \int_{t_{0}}^{t}(t-s)^{-\alpha}|u(s+h)-u(s)|_{X^{\alpha}} d s \\
& \leq \widetilde{K} h^{\beta}\left(t-t_{0}\right)^{-\beta}+C_{\alpha} K L \int_{t_{0}}^{t}|u(s+h)-u(s)|_{X^{\alpha}} d s,
\end{aligned}
$$

onde $\widetilde{K}=\beta^{-1} C_{1-\beta} C_{\beta} K\left(t_{1}-t_{0}\right)^{\beta-\alpha}\left|u\left(t_{0}\right)\right|_{X^{\alpha}}$. Portanto, a Desigualdade de Gronwall Singular, Lema 1.7.5, implica que existe uma constante $M \geq 0$ tal que

$$
|u(t+h)-u(t)|_{X^{\alpha}} \leq M h^{\beta}\left(t-t_{0}\right)^{-\beta},
$$

para todo $t \in\left(t_{0}, t_{1}\right), 0 \leq h \leq h_{0}$.

Afirmamos que, para todo $t \in\left(t_{0}, t_{1}\right)$,

$$
A \int_{t_{0}}^{t} e^{-A(t-s)} f(s) d s=\int_{t_{0}}^{t} A e^{-A(t-s)}[f(s)-f(t)] d s+f(t)-e^{-A\left(t-t_{0}\right)} f(t) .
$$

De fato, note que para $t \in\left(t_{0}, t_{1}\right)$ temos

$$
\int_{t_{0}}^{t} A e^{-A(t-s)} f(t) d s=\int_{t_{0}}^{t} \frac{d}{d s}\left(e^{-A(t-s)} f(t)\right) d s=f(t)-e^{-A\left(t-t_{0}\right)} f(t) .
$$

Logo, para $t \in\left(t_{0}, t_{1}\right)$,

$$
\int_{t_{0}}^{t} A e^{-A(t-s)} f(s) d s=\int_{t_{0}}^{t} A e^{-A(t-s)}[f(s)-f(t)] d s+f(t)-e^{-A\left(t-t_{0}\right)} f(t)
$$

e portanto temos

$$
\left|\int_{t_{0}}^{t} A e^{-A(t-s)} f(s) d s\right| \leq \int_{t_{0}}^{t} K(t-s)^{\beta-1}\left(t-t_{0}\right)^{-\beta} d s+B+B\left\|e^{-A\left(t-t_{0}\right)}\right\| .
$$


Como $0<\beta<1$, temos $\left|\int_{t_{0}}^{t} A e^{-A(t-s)} f(s) d s\right|$ define um número real. Como $A$ é um operador fechado, a igualdade (1.7.5) segue e afirmativa está demonstrada. Portanto, para $t \in\left(t_{0}, t_{1}\right)$ temos

$$
\begin{aligned}
\frac{d u}{d t} & =-A u(t)+f(t)=-A\left(e^{-A\left(t-t_{0}\right)} u\left(t_{0}\right)+\int_{t_{0}}^{t} e^{-A(t-s)} g(s) d s\right)+f(t) \\
& =-A e^{-A\left(t-t_{0}\right)} u\left(t_{0}\right)+e^{-A\left(t-t_{0}\right)} f(t)+\int_{t_{0}}^{t} A e^{-A\left(t-t_{0}\right)}[f(t)-f(s)] d s
\end{aligned}
$$

e

$$
\begin{aligned}
\left|\frac{d u}{d t}\right|_{X \gamma} & \leq C_{\gamma+1-\alpha} K\left(t-t_{0}\right)^{\alpha-\gamma-1}\left|u\left(t_{0}\right)\right|_{\alpha}+C_{\gamma} B\left(t-t_{0}\right)^{-\gamma} \\
& +\int_{t_{0}}^{t} C_{\gamma+1} K L(t-s)^{\beta-\gamma-1}\left(t-t_{0}\right)^{\beta} d s .
\end{aligned}
$$

É fácil ver que para $t \in\left(t_{0}, t_{1}\right)$

$$
\int_{t_{0}}^{t} C_{\gamma+1} K L(t-s)^{\beta-\gamma-1}\left(t-t_{0}\right)^{\beta} d s \leq\left(t-t_{0}\right)^{\alpha-\gamma-1} C_{\gamma+1} L\left(t-t_{0}\right)^{1-\alpha}(\beta-\gamma)^{-1}
$$

Portanto,

$$
\left|\frac{d u}{d t}\right|_{X^{\gamma}} \leq C\left(t-t_{0}\right)^{\alpha-\gamma-1}, \text { para } t \in\left(t_{0}, t_{1}\right],
$$

onde $C=\left\{C_{\gamma+1-\alpha}\left|u\left(t_{0}\right)\right|_{\alpha}+B C_{\gamma}\left(t_{1}-t_{0}\right)^{1-\alpha}+L C_{\gamma+1}(\beta-\gamma)^{-1}\left(t_{1}-t_{0}\right)^{1-\alpha}\right\}$ e isso conclui a demonstração. 


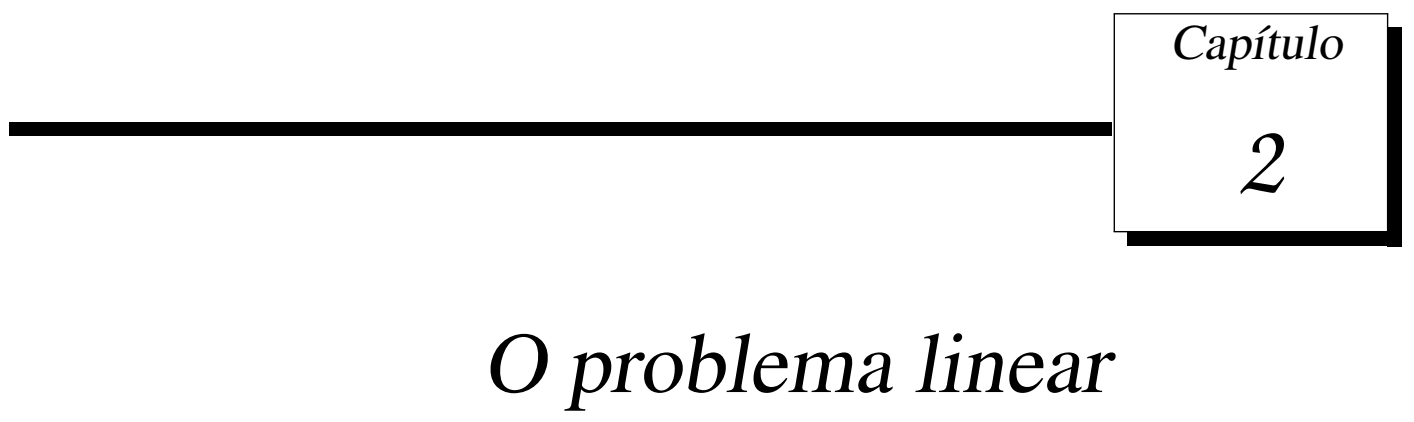

Neste capítulo analisamos o problema linear abstrato associado à equação (ERD). Na seção 2.3 apresentamos as hipóteses (HL1), (HL2) e (HL3). Com estas hipóteses o operador $u \mapsto-\mathrm{L} u+\beta(x) u$ apresentado na Introdução define um operador positivo auto-adjunto $\mathbf{A}: D(\mathbf{A}) \subset X \rightarrow X$, onde $X=L^{2}(\Omega)$. Nessa seção apresentamos as propriedades importantes do operador $\mathbf{A}$ e construímos uma família de operadores auto-adjuntos $\mathbf{A}_{(\alpha)}, \alpha \in \mathbb{R}$ tal que $\mathbf{A}_{(\alpha)}: X^{\alpha} \rightarrow X^{\alpha-1}$.

Para a construção do operador A necessitamos de alguns resultados auxiliares que são conhecidos na literatura, contudo as demonstrações não são encontradas fácilmente. Em [21] os autores reuniram estes fatos elementares na Proposição 2.2. Na seção 2.2 apresentamos a demonstração dessa proposição.

Iniciamos o capítulo demonstrando um resultado de análise funcional que será utilizado na Seção 2.3. A referência para este capítulo é o artigo [21].

\subsection{Um resultado de análise funcional}

Os lemas abaixo foram demonstrados na Seção 4 de [21].

Lema 2.1.1. Suponha que $\left(Y,\langle\cdot, \cdot\rangle_{Y}\right)$ e $\left(X,\langle\cdot, \cdot\rangle_{X}\right)$ sejam espaços de Hilbert tais que $Y \subset X, Y$ é denso em $\left(X,\langle\cdot, \cdot\rangle_{X}\right)$ e a inclusão $j:\left(Y,\langle\cdot, \cdot\rangle_{Y}\right) \rightarrow\left(X,\langle\cdot, \cdot\rangle_{X}\right)$ é contínua. Então para cada 
$u \in X$ existe um único $w_{u} \in Y$ tal que

$$
\left\langle v, w_{u}\right\rangle_{Y}=\langle v, u\rangle_{X} \text {, para todo } v \in Y \text {. }
$$

A aplicação $B: X \rightarrow X, u \mapsto w_{u}$ é linear, simétrica e positiva.

Demonstração. Para cada $u \in X$, a função $Y \ni v \mapsto\langle v, u\rangle_{X}$ é linear e contínua. Logo, pelo Teorema de Representação de Riesz existe um único $w_{u} \in Y$ tal que

$$
\langle v, u\rangle_{X}=\left\langle v, w_{u}\right\rangle_{Y}, \text { para todo } v \in Y
$$

Além disso do Teorema de Representação de Riesz segue que $B$ é linear. Para $u, v \in X$, note que

$$
\langle B u, v\rangle_{X}=\langle B u, B v\rangle_{Y}=\langle u, B v\rangle_{X}
$$

Logo $B$ é uma aplicação simétrica e, como $\langle B u, u\rangle_{X}=\langle B u, B u\rangle_{Y} \geq 0, B$ é também positiva.

Assuma a notação e as hipóteses do Lema 2.1.1. Seja $B^{1 / 2}$ a raiz quadrada de $B$, isto é, $B^{1 / 2}: X \rightarrow X$ é uma aplicação linear, simétrica e é tal que $B^{1 / 2} \circ B^{1 / 2}=B$.

Lema 2.1.2. Com a notação e as hipóteses do Lema 2.1.1 temos $B$ e $B^{1 / 2}$ são aplicaçães linereares injetoras e $R(B)$ é um conjunto denso em $Y$.

Demonstração. Se $u \in X$ é tal que $B u=0$, então

$$
0=\langle v, B u\rangle_{Y}=\langle v, u\rangle_{X}, \text { para todo } v \in Y
$$

Como $Y$ é denso em $X$, segue que $u=0$ e, portanto, $B$ é injetora. Analogamente mostramos que $B^{1 / 2}$ é injetora.

Para concluir a demonstração, seja $v \in Y$ tal que $\langle v, B u\rangle_{Y}=0$, para todo $u \in X$. Então $\langle v, u\rangle_{X}=0$, para todo $u \in X$ e então $v=0$, o que nos mostra que $R(B)$ é denso em $Y$.

Assuma a notação e as hipóteses do Lema 2.1.1. Considere $X^{1 / 2}=X_{B}^{1 / 2}=R\left(B^{1 / 2}\right) \mathrm{e}$ $B^{-1 / 2}: X^{1 / 2} \rightarrow X$ a aplicação inversa de $B^{1 / 2}$. É fácil ver que

$$
\langle u, v\rangle_{1 / 2}:=\left\langle B^{-1 / 2} u, B^{-1 / 2} v\right\rangle_{X}, u, v \in X^{1 / 2},
$$


define um produto interno em $X^{1 / 2}$. Mais ainda, $\left(X^{1 / 2},\langle\cdot, \cdot\rangle_{1 / 2}\right)$ é um espaço de Hilbert.

Lema 2.1.3. Com a notação e as hipóteses do Lema 2.1.1 temos $Y=X^{1 / 2} e\langle\cdot, \cdot\rangle_{Y}=\langle\cdot, \cdot\rangle_{1 / 2}$.

Demonstração. Afirmamos que $R(B)$ é um conjunto denso em $X^{1 / 2}$. De fato, como $B^{1 / 2}$ é uma aplicação simétrica, para $v \in X^{1 / 2}$ e $u \in X$, temos

$$
\langle v, B u\rangle_{1 / 2}=\left\langle B^{-1 / 2} v, B^{1 / 2} u\right\rangle_{X}=\langle v, u\rangle_{X}
$$

Logo, se $\langle v, B u\rangle_{1 / 2}=0$, para todo $u \in X$, segue que $v=0$ e nossa afirmativa está mostrada.

Agora afirmamos que

$$
\langle u, v\rangle_{Y}=\langle u, v\rangle_{1 / 2} \text {, para } u, v \in R(B) .
$$

De fato, se $u, v \in R(B)$, existem $\tilde{u}, \tilde{v} \in X$ tais que $u=B \tilde{u}$ e $v=B \tilde{v}$. Logo,

$$
\langle u, v\rangle_{Y}=\langle u, B \tilde{v}\rangle_{Y}=\langle u, \tilde{v}\rangle_{X}
$$

$\mathrm{e}$

$$
\langle u, v\rangle_{1 / 2}=\left\langle B^{1 / 2} \tilde{u}, B^{1 / 2} \tilde{v}\right\rangle_{X}=\langle B \tilde{u}, \tilde{v}\rangle_{X}=\langle u, \tilde{v}\rangle_{X},
$$

provando, assim, a afirmação.

A continuidade de $B^{1 / 2}: X \rightarrow X$ implica que para todo $u \in X^{1 / 2}$ temos

$$
|u|_{X} \leq\left\|B^{1 / 2}\right\|\left|B^{-1 / 2} u\right|_{X}=\left\|B^{1 / 2}\right\||u|_{1 / 2},
$$

e, portanto, a inclusão $i:\left(X^{1 / 2},|\cdot|_{1 / 2}\right) \rightarrow\left(X,|\cdot|_{X}\right)$ é contínua.

Seja $u \in Y$. Logo, existe uma sequência $\left(u_{n}\right)_{n}$ em $R(B)$ tal que $\left|u_{n}-u\right|_{Y} \rightarrow 0$ quando $n \rightarrow \infty$. Logo, $\left(u_{n}\right)_{n}$ é uma sequência de Cauchy em $Y$. A igualdade 2.1.1) implica que $\left(u_{n}\right)_{n}$ também é sequência de Cauchy em $X^{1 / 2}$. Logo, existe um $v \in X^{1 / 2}$ tal que $\left|u_{n}-v\right|_{X^{1 / 2}} \rightarrow 0$ quando $n \rightarrow \infty$. Como as inclusões $j:\left(Y,\langle\cdot, \cdot\rangle_{Y}\right) \rightarrow\left(X,\langle\cdot, \cdot\rangle_{X}\right)$ e $i:\left(X^{1 / 2},|\cdot|_{1 / 2}\right) \rightarrow\left(X,|\cdot|_{X}\right)$ são contínuas temos $\left|u_{n}-u\right|_{X} \rightarrow 0$ e $\left|u_{n}-v\right|_{X} \rightarrow 0$ quando $n \rightarrow \infty$. Portanto, $u=v$ e, assim, $u \in X^{1 / 2}$. Ou seja, $Y \subseteq X^{1 / 2}$. Pelo mesmo argumento obtemos $X^{1 / 2} \subseteq Y$.

Finalmente, a densidade de $R(B)$ em $Y$ e igualdade (2.1.1) implicam $\langle u, v\rangle_{1 / 2}=\langle u, v\rangle_{Y}$, para 
todo $u, v \in Y$.

\subsection{Resultados auxiliares sobre espaços de potências fra- cionárias}

Nesta seção demonstramos a Proposição 2.2 de [21].

Sejam $X$ um espaço de Banach e $A: D(A) \subset X \rightarrow X$ um operador setorial positivo. Logo $A$ é setorial em $X$, re $\sigma(A)>0$ e $-A$ é o gerador infinitesimal de um semigrupo analítico $\left\{e^{-A t} \mid\right.$ $t \geq 0\}$ de operadores lineares em $X$ (cf. Teorema 1.5.9.

Para $\alpha>0$ definimos, como usual, o operador $A^{-\alpha}: X \rightarrow X$ como

$$
A^{-\alpha} u=\frac{1}{\Gamma(\alpha)} \int_{0}^{\infty} t^{\alpha-1} e^{-A t} u d t, u \in X
$$

Denote por $X^{\alpha}=R\left(A^{-\alpha}\right), \alpha \geq 0$, a família de espaços de potências fracionárias em $X$ gerada por $A$. $X^{\alpha}$ é um espaço de Banach com a norma $|u|_{X^{\alpha}}:=\left|A^{\alpha} u\right|_{X}, u \in X^{\alpha}$.

Se $\beta>\alpha \geq 0$ então $X^{\beta}$ é denso em $X^{\alpha}$. Ainda mais,

$$
A^{-\alpha} A^{-\beta} x=A^{-\alpha-\beta} x, \quad \alpha, \beta \in(0, \infty), x \in X
$$

Seja agora $X$ um espaço de Hilbert e $A: D(A) \subseteq X \rightarrow X$ um operador auto-adjunto em $X$ com $\operatorname{re} \sigma(A)>0$. Então $A$ é setorial em $X$ e, para $\alpha \in(0, \infty)$,

$$
A^{-\alpha}=\int_{0}^{\infty} \lambda^{-\alpha} d E_{\lambda}
$$

onde $\left\{E_{\lambda}\right\}_{\lambda \in \mathbb{R}}$ é a medida espectral definida por $A$. Nesse caso, $X^{\alpha}$ é um espaço de Hilbert com respeito ao produto interno

$$
\langle u, v\rangle_{X^{\alpha}}:=\left\langle A^{\alpha} u, A^{\alpha} v\right\rangle_{X}, u, v \in X^{\alpha}
$$

Para $\alpha \in(0, \infty)$ seja $X^{-\alpha}=X_{A}^{-\alpha}$ o espaço dual de $X^{\alpha}$. Definimos em $X^{-\alpha}$ o produto interno 
$\langle\cdot, \cdot\rangle_{X^{-\alpha}}$ dual ao produto interno $\langle\cdot, \cdot\rangle_{X^{\alpha}}$, isto é,

$$
\left\langle u^{\prime}, v^{\prime}\right\rangle_{X^{-\alpha}}:=\left\langle R_{\alpha}^{-1} u^{\prime}, R_{\alpha}^{-1} v^{\prime}\right\rangle_{X^{\alpha}}, u^{\prime}, v^{\prime} \in X^{-\alpha},
$$

onde $R_{\alpha}: X^{\alpha} \rightarrow X^{-\alpha}$ é o isomorfismo de Fréchet-Riesz $u \mapsto\langle\cdot, u\rangle_{X^{\alpha}}$. O espaço de Hilbert $X^{-\alpha}$ é chamado espaço de potência fracionária de ordem $-\alpha$.

Denotemos por $\varphi$ a aplicação dualidade de $X$ em $X^{\prime}$, isto é,

$$
\varphi(x)=\langle\cdot, x\rangle, x \in X
$$

Lema 2.2.1. Sejam $\beta>0$ e $x \in X$ e defina o funcional linear $f_{x}: X^{\beta} \rightarrow \mathbb{R}$ dado por

$$
f_{x}(y)=\langle y, x\rangle_{X}, \text { para todo } y \in X^{\beta}
$$

Então $f_{x} \in X^{-\beta}$.

Demonstração. Sejam $\beta>0$ e $x \in X$ fixados. Claramente $f_{x}$ é linear e está bem definida.

Mostremos que $f_{x}$ é limitada de $X^{\beta}$. De fato, como $X^{\beta}$ está continuamente imerso em $X$, existe constante $B \geq 0$ tal que

$$
|y|_{X} \leq B|y|_{X^{\beta}} \text {, para todo } y \in X^{\beta}
$$

Portanto, se $y \in X^{\beta}$,

$$
f_{x}(y)=\langle y, x\rangle_{X} \leq|y|_{X}|x|_{X} \leq B|x|_{X}|y|_{X^{\beta}} .
$$

Concluindo que $f_{x}$ é limitada em $X^{\beta}$ e $\left\|f_{x}\right\| \leq B|x|_{X}$.

Sejam $\alpha, \beta \in \mathbb{R}$ arbitrários. Nosso objetivo é definir aplicações lineares contínuas entre os espaços $X^{\alpha}$ e $X^{\beta}$.

(i) Se $\beta \geq \alpha \geq 0$, defina $\varphi_{\beta, \alpha}: X^{\beta} \rightarrow X^{\alpha}$ como sendo a aplicação inclusão.

(ii) Se $\beta \geq \alpha>0$, defina então a aplicação $\varphi_{-\alpha,-\beta}: X^{-\alpha} \rightarrow X^{-\beta}$ por $\varphi_{-\alpha,-\beta}\left(y^{\prime}\right)=\left.y^{\prime}\right|_{X^{\beta}}$, para $y^{\prime} \in X^{-\alpha}$. 
(iii) Se $\beta>0$, defina $\varphi_{0,-\beta}: X^{0}=X \rightarrow X^{-\beta}$ por $\varphi_{0,-\beta}(x)=f_{x}$, onde $f_{x} \in X^{-\beta}$ é como no Lema 2.2.1.

(iv) Finalmente, se $\alpha>0$ e $\beta>0$, então $\varphi_{\beta,-\alpha}:=\varphi_{0,-\alpha} \circ \varphi_{\beta, 0}$.

Apresentamos a seguir diversas propriedades das aplicações que acabamos de definir.

Proposição 2.2.2. Para todo $\alpha, \beta \in \mathbb{R} \operatorname{com} \beta \geq \alpha$ a aplicação $\varphi_{\beta, \alpha}: X^{\beta} \rightarrow X^{\alpha}$ está bem definida, é linear, limitada e injetora. O subespaço $\varphi_{\beta, \alpha}\left(X^{\beta}\right)$ é denso no espaço de Hilbert $X^{\alpha}$.

Demonstração. Sejam $\alpha, \beta \in \mathbb{R} \operatorname{com} \beta \geq \alpha$. É claro que a aplicação $\varphi_{\beta, \alpha}: X^{\beta} \rightarrow X^{\alpha}$ está bem definida e é linear.

Afirmamos que $\varphi_{\beta, \alpha}$ é uma aplicação limitada, injetora e sua imagem é um conjunto denso em $X^{\alpha}$. Para mostrar este fato teremos que considerar três casos.

\section{Primeiro Caso:}

Suponha $\beta \geq \alpha \geq 0$. Neste caso a aplicação $\varphi_{\beta, \alpha}$ é a inclusão e a injetividade é clara, assim como a continuidade. Ainda, $X^{\beta}$ é denso em $X^{\alpha}, \operatorname{logo} \varphi_{\beta, \alpha}\left(X^{\beta}\right)$ é denso em $X^{\alpha}$.

\section{Segundo Caso:}

Suponha $\alpha<0 \leq \beta$. Logo $\varphi_{\beta, \alpha}=\varphi_{0, \alpha} \circ \varphi_{\beta, 0}$. Segue do Primeiro Caso que $\varphi_{\beta, 0}$ é limitada, injetora e sua imagem é densa em $X^{0}=X$. Portanto, resta mostrarmos que $\varphi_{0, \alpha}: X \rightarrow X^{\alpha}$ também é uma aplicação limitada, injetora e possui imagem densa em $X^{\alpha}$. Recordemos que dado $x \in X, \varphi_{0, \alpha}(x): X^{-\alpha} \rightarrow \mathbb{R}(-\alpha>0)$ é dado por $\varphi_{0, \alpha}(x)(y)=\langle y, x\rangle_{X}$, para $y \in X^{-\alpha}$.

Para $x \in X$, pelo Teorema de Hahn-Banach sabemos que

$$
\left\|f_{x}\right\|=\sup _{\substack{w \in X^{-\alpha} \\|w|_{X^{-\alpha}}=1}} f_{x}(w)=\sup _{\substack{w \in X^{-\alpha} \\|w|_{X^{-\alpha}}=1}}\langle w, x\rangle_{X}
$$

Logo

$$
\left\|f_{X}\right\| \leq \sup _{\substack{w \in X^{-\alpha} \\|w|_{X^{-\alpha}}=1}}|w|_{X}|x|_{X} \leq \sup _{\substack{w \in X^{-\alpha} \\|w|_{X^{-\alpha}}=1}} B|w|_{X^{-\alpha}}|x|_{X} \leq B|x|_{X} .
$$

Portanto a aplicação $X \ni x \mapsto f_{x} \in X^{\alpha}$ é limitada. 
Seja $x \in X$ e suponha que $\varphi_{0, \alpha}(x)=0 \in X^{\alpha}$. Logo,

$$
\langle y, x\rangle_{X}=0 \text { para todo } y \in X^{-\alpha}
$$

Como $X^{-\alpha}$ é denso em $X$, segue que $x=0$. Portanto, $\varphi_{0, \alpha}$ é injetora.

Seja, agora, $z \in X^{-\alpha}$ tal que $\varphi_{0, \alpha}(w)(z)=f_{w}(z)=0$, para todo $w \in X$. A fim de mostrar que $\varphi_{0, \alpha}(X)$ é denso em $X^{\alpha}$, devemos mostrar que $z=0$. De fato, apenas note que $f_{w}(z)=$ $\langle z, w\rangle_{X}=0$, para todo $w \in X$ e, portanto, $z=0$. Como era desejado.

\section{Terceiro caso:}

Suponha, por fim, que $\alpha \leq \beta<0$. Temos que $\varphi_{\beta, \alpha}: X^{\beta} \rightarrow X^{\alpha}$ é a restrição de funcionais definidos em $X^{-\beta}$ a $X^{-\alpha}$ (notemos que $-\beta>0$ e $-\alpha>0$ ).

Dado $x^{*} \in X^{\beta}$, pelo Teorema de Fréchet-Riesz existe um $x \in X^{-\beta}$ tal que

$$
x^{*}(y)=\langle y, x\rangle_{X^{-\beta}} \text {, para todo } y \in X^{-\beta}
$$

Logo, para $w \in X^{-\alpha}, \varphi_{\beta, \alpha}\left(x^{*}\right)(w)=\langle w, x\rangle_{X^{-\beta}}$. E, analogamente ao Segundo Caso, teremos $\varphi_{\beta, \alpha}$ limitada de $X^{\beta}$ em $X^{\alpha}$.

Mostremos que $\varphi_{\beta, \alpha}$ é injetora. De fato, seja $x \in X^{\beta}$ tal que $\varphi_{\beta, \alpha}(x)=0 \in X^{\alpha}$. Isto é,

$$
\varphi_{\beta, \alpha}(x)(v)=x(v)=0, \text { para todo } v \in X^{-\alpha} .
$$

Como $X^{-\alpha}$ é denso em $X^{-\beta}$, segue que $x=0$.

Finalmente, seja $v \in X^{-\alpha}$ tal que $\varphi_{\beta, \alpha}(x)(v)=0$, para todo $x \in X^{\beta}$. Analogamente ao caso anterior devemos mostrar que $v=0$. Porém, note que

$$
\varphi_{\beta, \alpha}(x)(v)=\left.x\right|_{X^{-\alpha}} v=x(v),
$$

uma vez que $v \in X^{-\alpha}$. Portanto, como $X^{-\alpha}$ é um subespaço denso de $X^{-\beta}$, 2.2.2) implica que $x \equiv 0$, concluindo a demonstração. 
Proposição 2.2.3. Para todo $\alpha, \beta \in \mathbb{R} \operatorname{com} \beta \geq \alpha$ a aplicação $\varphi_{\beta, \alpha}: X^{\beta} \rightarrow X^{\alpha}$ satisfaz

$$
\begin{aligned}
\varphi_{\alpha, \alpha} & =I_{X^{\alpha}}, \text { para todo } \alpha \in \mathbb{R} e \\
\varphi_{\gamma, \alpha} & =\varphi_{\beta, \alpha} \circ \varphi_{\gamma, \beta}, \text { para } \alpha, \beta, \gamma \in \mathbb{R} \operatorname{com} \gamma \geq \beta \geq \alpha .
\end{aligned}
$$

Demonstração. É fácil ver que $\varphi_{\alpha, \alpha}=I_{X^{\alpha}}$, para qualquer $\alpha \in \mathbb{R}$. Sejam $\alpha, \beta, \gamma \in \mathbb{R}$ com $\gamma \geq \beta \geq \alpha$. A demonstração de que $\varphi_{\gamma, \alpha}=\varphi_{\beta, \alpha} \circ \varphi_{\gamma, \beta}$ será dividada em cinco casos.

Primeiro Caso: $\alpha \geq 0$.

Todas as funções em questão são inclusões, desse modo fica claro que $\varphi_{\gamma, \alpha}=\varphi_{\beta, \alpha} \circ \varphi_{\gamma, \beta}$.

Segundo Caso: $\gamma<0$.

Todas as funções em questão são restrições de funcionais lineares a subespaços encaixados, isto é, $X^{-\gamma} \supset X^{-\beta} \supset X^{-\alpha}$ e $\varphi_{\gamma, \alpha} f=\left.f\right|_{X^{-\alpha}}$. Por outro lado, $\varphi_{\beta, \alpha} \circ \varphi_{\gamma, \beta} f=\left.\varphi_{\beta, \alpha} f\right|_{X^{-\beta}}=\left.f\right|_{X-\alpha}$. $\operatorname{Logo} \varphi_{\gamma, \alpha}=\varphi_{\beta, \alpha} \circ \varphi_{\gamma, \beta}$.

Terceiro Caso: $\beta \geq 0$ e $\alpha<0$.

Segue da definição que $\varphi_{\gamma, \alpha}=\varphi_{0, \alpha} \circ \varphi_{\gamma, 0}$ e $\varphi_{\beta, \alpha}=\varphi_{0, \alpha} \circ \varphi_{\beta, 0}$. Logo,

$$
\begin{aligned}
\varphi_{\beta, \alpha} \circ \varphi_{\gamma, \beta} & =\varphi_{0, \alpha} \circ \varphi_{\beta, 0} \circ \varphi_{\gamma, \beta} \\
& =\varphi_{0, \alpha} \circ \varphi_{\gamma, 0}=\varphi_{\gamma, \alpha}
\end{aligned}
$$

onde, na segunda igualdade, usamos o resultado para $\gamma \geq \beta \geq 0$.

Quarto Caso: $\gamma=0$ e $\beta<0$.

Note que $\varphi_{\beta, \alpha}$ é a restrição de funcionais lineares e, para $y \in X^{-\beta}, \varphi_{0, \beta}(x)(y)=\langle y, x\rangle_{X}$, $x \in X$. Logo, sua restrição a $X^{\alpha}$ é exatamente $\varphi_{0, \alpha}$.

Quinto Caso: $\gamma>0$ e $\beta<0$.

Segue da definição que $\varphi_{\gamma, \beta}=\varphi_{0, \beta} \circ \varphi_{\gamma, 0}$ e $\varphi_{\gamma, \alpha}=\varphi_{0, \alpha} \circ \varphi_{\gamma, 0}$. Logo,

$$
\begin{aligned}
\varphi_{\beta, \alpha} \circ \varphi_{\gamma, \beta} & =\varphi_{\beta, \alpha} \circ \varphi_{0, \beta} \circ \varphi_{\gamma, 0} \\
& =\varphi_{0, \alpha} \circ \varphi_{\gamma, 0}=\varphi_{\gamma, \alpha}
\end{aligned}
$$


onde, na segunda igualdade, usamos o resultado para $0>\beta \geq \alpha$.

Proposição 2.2.4. Para todo $\alpha, \gamma \in \mathbb{R}, \theta \in[0,1]$ com $\alpha \leq \gamma$ e $\beta=(1-\theta) \alpha+\theta \gamma$ vale a desigualdade de interpolação:

$$
\left|\varphi_{\gamma, \beta} x\right|_{X^{\beta}} \leq\left|\varphi_{\gamma, \alpha} x\right|_{X^{\alpha}}^{1-\theta}|x|_{X^{\gamma}}^{\theta} \text {, para todo } x \in X^{\gamma}
$$

Demonstração. Combinando as Proposições 1.5.23 e 1.5.27 obtemos

$$
\left|A^{\beta} x\right|_{X} \leq\left|A^{\alpha} x\right|_{X}^{1-\theta}\left|A^{\gamma} x\right|_{X}^{\theta}, \text { para todo } x \in X^{\delta}
$$

e todo $\alpha, \gamma \in \mathbb{R}, \delta \geq 0, \theta \in[0,1]$, com $\alpha \leq \gamma \leq \delta$ e $\beta=(1-\theta) \alpha+\theta \gamma$.

Afirmamos que para todo $\alpha>0, \beta \geq 0$

$$
\left|\varphi_{\beta, \beta-\alpha} A^{-\beta} x\right|_{X^{\beta-\alpha}}=\left|A^{-\alpha} x\right|_{X}, \text { para todo } x \in X
$$

De fato, sejam $\alpha>0$ e $\beta \geq 0$. Suponha que $\beta \geq \alpha$. Temos

$$
\left|\varphi_{\beta, \beta-\alpha} A^{-\beta} x\right|_{X-\alpha}=\left|A^{\beta-\alpha} \varphi_{\beta, \beta-\alpha} A^{-\beta} x\right|_{X}=\left|A^{-\alpha} x\right|_{X} \text {, para todo } x \in X
$$

Suponha agora que $\beta<\alpha$. Note que, pela definição de $\varphi_{0, \beta-\alpha}$, temos $R_{\beta-\alpha}^{-1}\left(\varphi_{\beta, \beta-\alpha} A^{-\beta} x\right)=$ $A^{\beta-2 \alpha} x$ para todo $x \in X$ e portanto

$$
\begin{aligned}
\left|\varphi_{\beta, \beta-\alpha} A^{-\beta} x\right|_{X^{\beta-\alpha}} & =\left|A^{\alpha-\beta} R_{\alpha-\beta}^{-1} \varphi_{\beta, \beta-\alpha} A^{-\beta} x\right|_{X} \\
& =\left|A^{\alpha-\beta} A^{\beta-2 \alpha} x\right|_{X} \\
& =\left|A^{-\alpha} x\right|_{X},
\end{aligned}
$$

para todo $x \in X$. A igualdade 2.2.5) está demonstrada.

Em particular, se $\beta=0$ em 2.2 .5 , obtemos

$$
\left|\varphi_{\delta, \alpha} x\right|_{X^{\alpha}}=\left|A^{\alpha} x\right|_{X}, \text { para todo } x \in X^{\delta}
$$

e todo $\alpha \in \mathbb{R}, \delta \in[0, \infty)$ com $\delta \geq \alpha$. Agora, a Proposição 2.2.2 e as equações 2.2.4) e 2.2.6 
demonstram que para todo $\alpha, \gamma \in \mathbb{R}, \theta \in[0,1]$ e $\beta=(1-\theta) \alpha+\theta \gamma$ temos:

$$
\left|\varphi_{\gamma, \beta} x\right|_{X^{\beta}} \leq\left|\varphi_{\gamma, \alpha} x\right|_{X^{\alpha}}^{1-\theta}|x|_{X^{\gamma}}^{\theta}, \text { para todo } x \in X^{\gamma}
$$

A proposição está demonstrada.

Para cada $\alpha \geq 0$ e $\beta \geq 0$ defina a aplicação

$$
A_{(\alpha)}^{-\beta}:=\left.A^{-\beta}\right|_{X^{\alpha}}: X^{\alpha} \rightarrow X^{\beta+\alpha}
$$

Proposição 2.2.5. Com a notação introduzida acima, para todo $\alpha \geq 0$ e $\beta \geq 0$ a aplicação $A_{(\alpha)}^{-\beta}$ é uma isometria linear bijetora.

Demonstração. É claro que $A_{(\alpha)}^{-\beta}$ é uma aplicação linear e injetora. Mostremos que é sobrejetora. Seja $y \in X^{\beta+\alpha}$. Logo existe um $x \in X$ tal que $A^{-\beta-\alpha} x=y$. Em particular, segue de 2.2.1 que $A^{-\beta} A^{-\alpha} x=y$. Considere $z:=A^{-\alpha} x \in X^{\alpha}$ e teremos $A_{(\alpha)}^{-\beta} z=A^{-\beta} A^{-\alpha} x=y$. Portanto $A_{(\alpha)}^{-\beta}$ é sobrejetora.

Note que, se $y \in X^{\alpha}$, então

$$
\left|A^{-\beta} y\right|_{X^{\beta+\alpha}}=\left|A^{-(\beta+\alpha)} A^{\alpha} y\right|_{X^{\beta+\alpha}}=\left|A^{\alpha} y\right|_{X}=|y|_{X^{\alpha}}
$$

ou seja, $A_{(\alpha)}^{-\beta}$ é uma isometria.

Para provar o próximo resultado necessitaremos de um fato auxiliar que é simples e conhecido cuja demonstração não será apresentada.

Lema 2.2.6. Sejam $E_{k}, F_{k}, k \in\{1,2\}$ espaços vetoriais (sobre $\mathbb{R}$ ou $\mathbb{C}$ ) com $E_{2}$ e $F_{2}$ completos. Suponha que e $: E_{1} \rightarrow E_{2}, f: F_{1} \rightarrow F_{2}$ e $B_{1}: E_{1} \rightarrow F_{1}$ sejam isometrias lineares com $B_{1}$ bijetora. Se o conjunto e $\left(E_{1}\right)$ é denso em $E_{2}$ e o conjunto $f\left(F_{1}\right)$ é denso em $F_{2}$ então existe uma única isometria linear bijetora $B_{2}: E_{2} \rightarrow F_{2}$ tal que $B_{2} \circ e=f \circ B_{1}$.

Proposição 2.2.7. Para todo $\alpha>0$ e $\beta \geq 0$ existe uma única isometria linear bijetora $A_{(-\alpha)}^{-\beta}: X^{-\alpha} \rightarrow X^{\beta-\alpha}$ tal que

$$
A_{(-\alpha)}^{-\beta} \circ \varphi_{0,-\alpha}=\varphi_{\beta, \beta-\alpha} \circ A^{-\beta}
$$


Demonstração. Se $\beta>0$, considere em $X^{\beta}$ a norma $\eta_{\beta-\alpha}(\cdot)$ dada por $\eta_{\beta-\alpha}(x)=\left|A^{\beta-\alpha} x\right|_{X}$, $x \in X^{\beta}$. Observamos que, em geral, $\left(X^{\beta}, \eta_{\beta-\alpha}(\cdot)\right)$ não é um espaço de Banach. Fórmula 2.2.1) implica que $\varphi_{\beta, \beta-\alpha}: X^{\beta} \rightarrow X^{\beta-\alpha}$ é uma isometria entre $\left(X^{\beta}, \eta_{\beta-\alpha}(\cdot)\right)$ e o espaço de Hilbert $\left(X^{\beta-\alpha},|\cdot|_{X^{\beta-\alpha}}\right)$.

Se $\beta=0$, considere em $X$ a norma $\eta_{-\alpha}(\cdot)$ dada por $\eta_{-\alpha}(x)=\left|A^{-\alpha} x\right|_{X}, x \in X$. Novamente, temos $\varphi_{0,-\alpha}: X \rightarrow X^{-\alpha}$ isometria entre $\left(X, \eta_{-\alpha}(\cdot)\right)$ e o espaço de Hilbert $\left(X^{-\alpha},|\cdot|_{X^{-\alpha}}\right)$.

Assim, como $A^{-\beta}$ é uma isometria bijetora de $X$ munido com a norma $\eta_{-\alpha}$ em $X^{\beta-\alpha}$ munido com a norma $\eta_{\beta-\alpha}$, aplicando o Lema 2.2.6 com $E_{1}=X$ (munido com a norma $\eta_{-\alpha}$ ), $E_{2}=X^{-\alpha}, F_{1}=X^{\beta}$ (munido com a norma $\eta_{\beta-\alpha}$ ), $F_{2}=X^{\beta-\alpha}, B_{1}=A^{-\beta}, e=\varphi_{0,-\alpha}$ e $f=\varphi_{\beta, \beta-\alpha}$, segue que existe uma única isometria linear bijetora $B_{2}:=A_{(-\alpha)}^{-\beta}$ tal que $A_{(-\alpha)}^{-\beta} \circ \varphi_{0,-\alpha}=\varphi_{\beta, \beta-\alpha} \circ A^{-\beta}$.

Dados $\gamma \in \mathbb{R}$ e $\beta>0$, segue das Proposições 2.2.5 e 2.2.7 que está bem definida uma isometria linear bijetora $A_{(\gamma)}^{-\beta}: X^{\gamma} \rightarrow X^{\beta+\gamma}$. No caso em que $\gamma \geq 0, A_{(\gamma)}^{-\beta}$ é a restrição de $A^{-\beta}$ a $X^{\gamma}$. Se $\gamma<0$, então $A_{(\gamma)}^{-\beta}$ é tal que $A_{(\gamma)}^{-\beta} \circ \varphi_{0, \gamma}=\varphi_{\beta, \beta+\gamma} \circ A^{-\beta}$.

Quando $\beta>0$ e $\alpha \in \mathbb{R}$, definimos a aplicação $A_{(\alpha)}^{\beta}: X^{\alpha} \rightarrow X^{-\beta+\alpha}$ por

$$
A_{(\alpha)}^{\beta}=\left(A_{(-\beta+\alpha)}^{-\beta}\right)^{-1}
$$

Denote por $A_{(\alpha)}:=A_{(\alpha)}^{1}$.

Proposição 2.2.8. Com a notação introduzida acima, para todo $\gamma, \gamma^{\prime} \in \mathbb{R}$, com $\gamma>\gamma^{\prime}$, e todo $\beta \in \mathbb{R}$

$$
\varphi_{-\beta+\gamma,-\beta+\gamma^{\prime}} \circ A_{(\gamma)}^{\beta}=A_{\left(\gamma^{\prime}\right)}^{\beta} \circ \varphi_{\gamma, \gamma^{\prime}}
$$

Demonstração. Sejam $\gamma, \gamma^{\prime} \in \mathbb{R}$ tais que $\gamma>\gamma^{\prime}$ e $\beta \in \mathbb{R}$. Suponhamos, primeiramente, que $\beta \leq 0$ e note que, neste caso $A_{\left(\gamma^{\prime}\right)}^{\beta} \circ \varphi_{\gamma, \gamma^{\prime}}=A_{\left(\gamma^{\prime}\right)}^{\beta} \circ \varphi_{\gamma, \gamma^{\prime}}$. Consideremos três possibilidades:

Primeiro Caso: $\gamma \geq 0>\gamma^{\prime}$.

Como $\gamma^{\prime}<0$, temos que $A_{\left(\gamma^{\prime}\right)}^{\beta} \circ \varphi_{0, \gamma^{\prime}}=\varphi_{-\beta,-\beta+\gamma^{\prime}} \circ A^{\beta}$. Além disso, como $\gamma \geq 0$, temos 
$\varphi_{-\beta+\gamma,-\beta}^{-1} \circ A^{\beta} \circ \varphi_{\gamma, 0}=A_{(\gamma)}^{\beta}$. Portanto,

$$
\begin{aligned}
A_{\left(\gamma^{\prime}\right)}^{\beta} \circ \varphi_{\gamma, \gamma^{\prime}} & =\varphi_{-\beta,-\beta+\gamma^{\prime}} \circ A^{\beta} \circ \varphi_{\gamma, 0} \\
& =\varphi_{-\beta+\gamma, \beta+\gamma^{\prime}} \circ \varphi_{-\beta+\gamma,-\beta}^{-1} A^{\beta} \circ \varphi_{\gamma, 0} \\
& =\varphi_{-\beta+\gamma,-\beta+\gamma^{\prime}} \circ A_{(\gamma)}^{\beta} .
\end{aligned}
$$

Segundo Caso: $0>\gamma$.

Para mostrarmos a igualdade desejada basta verificarmos que

$$
\varphi_{-\beta+\gamma,-\beta+\gamma^{\prime}} \circ A_{(\gamma)}^{\beta} \circ \varphi_{\gamma, \gamma^{\prime}}^{-1} \circ \varphi_{0, \gamma^{\prime}}=\varphi_{-\beta,-\beta+\gamma^{\prime}} \circ A^{\beta}
$$

pois a unicidade da aplicação $A_{\left(\gamma^{\prime}\right)}^{\beta}$ implicará o resultado. Observe que $\varphi_{0, \gamma^{\prime}}=\varphi_{\gamma, \gamma^{\prime}} \circ \varphi_{0, \gamma} \mathrm{e}$

$$
\begin{aligned}
\varphi_{-\beta+\gamma,-\beta+\gamma^{\prime}} \circ A_{(\gamma)}^{\beta} \circ \varphi_{\gamma, \gamma^{\prime}}^{-1} \circ \varphi_{0, \gamma^{\prime}} & =\varphi_{-\beta+\gamma,-\beta+\gamma^{\prime}} \circ A_{(\gamma)}^{\beta} \circ \varphi_{0, \gamma} \\
& =\varphi_{-\beta+\gamma,-\beta+\gamma^{\prime}} \varphi_{-\beta,-\beta+\gamma} \circ A^{\beta} \\
& =\varphi_{-\beta,-\beta+\gamma^{\prime}} \circ A^{\beta},
\end{aligned}
$$

como era desejado.

Terceiro Caso: $\gamma^{\prime} \geq 0$

Como $-\beta \geq 0$ as aplicações $\varphi_{\gamma, \gamma^{\prime}}$ e $\varphi_{-\beta+\gamma,-\beta+\gamma^{\prime}}$ são inclusões. Portanto, se $x \in X^{\gamma}$, por um lado

$$
A_{\left(\gamma^{\prime}\right)}^{\beta} \circ \varphi_{\gamma, \gamma^{\prime}}(x)=A^{\beta} x \in X^{\gamma^{\prime}-\beta}
$$

Por outro lado $\varphi_{-\beta+\gamma,-\beta+\gamma^{\prime}} \circ A_{(\gamma)}^{\beta}(x)=A^{\beta} x \in X^{\gamma^{\prime}-\beta}$ e segue a igualdade desejada.

Para completar a demonstração, suponhamos que $\beta>0$. Neste caso, $A_{(\gamma)}^{\beta}=\left(A_{(-\beta+\gamma)}^{-\beta}\right)^{-1}$. Como $-\beta+\gamma>-\beta+\gamma^{\prime}$, temos

$$
\varphi_{\gamma, \gamma^{\prime}} \circ A_{(-\beta+\gamma)}^{-\beta}=A_{\left(-\beta+\gamma^{\prime}\right)}^{-\beta} \circ \varphi_{-\beta+\gamma,-\beta+\gamma^{\prime}}
$$


Portanto suas inversas são iguais e temos

$$
\left(A_{(-\beta+\gamma)}^{-\beta}\right)^{-1} \circ \varphi_{\gamma, \gamma^{\prime}}^{-1}=\varphi_{-\beta+\gamma,-\beta+\gamma^{\prime}}^{-1} \circ\left(A_{\left(-\beta+\gamma^{\prime}\right)}^{-\beta}\right)^{-1}
$$

$\operatorname{Logo}, \varphi_{-\beta+\gamma,-\beta+\gamma^{\prime}} \circ A_{(-\beta+\gamma)}^{\beta}=A_{\left(\gamma^{\prime}\right)}^{\beta} \circ \varphi_{\gamma, \gamma^{\prime}}$

Para demonstrar o próximo resultado novamente dividiremos a demonstração em casos. Em cada caso, teremos que fazer novas divisões o que tornará a demonstração um tanto quanto técnica.

Proposição 2.2.9. Com a notação introduzida acima, para todo $\alpha, \beta, \gamma \in \mathbb{R}$

$$
A_{(-\gamma+\alpha)}^{\beta} \circ A_{(\alpha)}^{\gamma}=A_{(\alpha)}^{\beta+\gamma}
$$

Demonstração. Dividiremos a demonstração em quatro casos.

Primeiro Caso: $\beta \leq 0$ e $\gamma \leq 0$.

Suponha que $\alpha>0$. Com isso temos

$$
A_{(\alpha)}^{\beta+\gamma} x=A^{\beta+\gamma} x \in X^{\alpha-\beta-\gamma} \text {, para todo } x \in X^{\alpha} .
$$

Por outro lado,

$$
A_{(\alpha)}^{\gamma} x=A^{\gamma} x \in X^{\alpha-\gamma}, \text { para todo } x \in X^{\alpha}
$$

$\mathrm{e}, \operatorname{assim}, A_{(-\gamma+\alpha)}^{\beta} A^{\gamma} x=A^{\beta}\left(A^{\gamma} x\right)=A^{\beta+\gamma} x \in X^{\alpha-\beta-\gamma}$.Portanto $A_{(-\gamma+\alpha)}^{\beta} \circ A_{(\alpha)}^{\gamma}=A_{(\alpha)}^{\beta+\gamma}$.

Suponha que $\alpha \leq 0$ com $-\gamma+\alpha>0$. Segue da Proposição 2.2.7 que

$$
\begin{aligned}
& A_{(\alpha)}^{\gamma} \circ \varphi_{0, \alpha}=\varphi_{-\gamma,-\gamma+\alpha} \circ A^{\gamma}, \\
& A_{(\alpha)}^{\beta+\gamma} \circ \varphi_{0, \alpha}=\varphi_{-\beta-\gamma,-\beta-\gamma+\alpha} \circ A^{\beta+\gamma} .
\end{aligned}
$$

Além disso, $A_{(-\gamma+\alpha)}^{\beta}$ é a restrição de $A^{\beta}$ a $X^{-\gamma+\alpha} \subset X$ e $A_{(-\gamma)}^{\beta}$ é a restrição de $A^{\beta}$ a $X^{-\gamma} \subset X$ 
(ver fórmula (2.2.7)). Portanto,

$$
\begin{aligned}
A_{(-\gamma+\alpha)}^{\beta} \circ A_{(\alpha)}^{\gamma} \circ \varphi_{0, \alpha} & =A_{-\gamma+\alpha}^{\beta} \varphi_{-\gamma,-\gamma+\alpha} \circ A^{\gamma} \\
& =\varphi_{-\beta-\gamma,-\beta-\gamma+\alpha} \circ A_{(-\gamma)}^{\beta} \circ A^{\gamma} \\
& =\varphi_{-\beta-\gamma,-\beta-\gamma+\alpha} \circ A^{\beta+\gamma} .
\end{aligned}
$$

e isso implica a desigualdade desejada.

Para completar a demonstração do Primeiro Caso, suponha que $\alpha \leq 0$ com $-\gamma+\alpha \leq 0$. Proposição 2.2.7 implica que

$$
\begin{aligned}
A_{(-\gamma+\alpha)}^{\beta} \circ \varphi_{0,-\gamma+\alpha} & =\varphi_{-\beta,-\beta-\gamma+\alpha} \circ A^{\beta} \\
A_{(\alpha)}^{\gamma} \circ \varphi_{0, \alpha} & =\varphi_{-\gamma,-\gamma+\alpha} \circ A^{\gamma} \\
A_{(\alpha)}^{\beta+\gamma} \circ \varphi_{0, \alpha} & =\varphi_{-\beta-\gamma,-\beta-\gamma+\alpha} \circ A^{\beta+\gamma} .
\end{aligned}
$$

Como $-\beta-\gamma \geq-\beta \geq-\beta-\gamma+\alpha$, temos $\varphi_{-\beta-\gamma,-\beta-\gamma+\alpha}=\varphi_{-\beta,-\beta-\beta+\alpha} \varphi_{-\beta-\gamma,-\beta}$. As aplicações $\varphi_{-\gamma, 0}$ e $\varphi_{-\beta-\gamma,-\beta}$ são inclusões e portanto:

$$
\begin{aligned}
A_{(-\gamma+\alpha)}^{\beta} \circ A_{(\alpha)}^{\gamma} \circ \varphi_{0, \alpha} & =A_{(-\gamma+\alpha)}^{\beta} \circ \varphi_{-\gamma,-\gamma+\alpha} \circ A^{\gamma} \\
& =A_{(-\gamma+\alpha)}^{\beta} \circ \varphi_{0,-\gamma+\alpha} \circ \varphi_{-\gamma, 0} \circ A^{\gamma} \\
& =\varphi_{-\beta,-\beta-\gamma+\alpha} \circ A^{\beta} \circ \varphi_{-\gamma, 0} \circ A^{\gamma} \\
& =\varphi_{-\beta-\gamma,-\beta-\gamma+\alpha} \circ \varphi_{-\beta-\gamma,-\beta}^{-1} \circ A^{\beta} \circ \varphi_{-\gamma, 0} \circ A^{\gamma} \\
& =\varphi_{-\beta-\gamma,-\beta-\gamma+\alpha} \circ A^{\beta+\gamma}
\end{aligned}
$$

e isso implica a desigualdade desejada.

Segundo Caso: $\beta>0, \gamma>0$ e $\alpha \in \mathbb{R}$.

Utilizando a definição descrita na fórmula (2.2.9) temos

$$
\begin{aligned}
A_{(-\gamma+\alpha)}^{\beta} & =\left(A_{-\beta-\gamma+\alpha}^{-\beta}\right)^{-1}, \\
A_{(\alpha)}^{\gamma} & =\left(A_{(-\gamma+\alpha)}^{-\gamma}\right)^{-1}, \\
A_{(\alpha)}^{\beta+\gamma} & =\left(A_{-\beta-\gamma+\alpha}^{-\beta-\gamma}\right)^{-1} .
\end{aligned}
$$


Defina $\widetilde{\alpha}=-\beta-\gamma+\alpha, \widetilde{\beta}=-\gamma$ e $\widetilde{\gamma}=-\beta$. Segue que $\widetilde{\beta} \leq 0$ e $\widetilde{\gamma} \leq 0$ e aplicando a igualdade obtida no Primeiro Caso temos

$$
A_{(-\gamma+\alpha)}^{-\gamma} \circ A_{(-\beta-\gamma+\alpha)}^{-\beta}=A_{(-\beta-\gamma+\alpha)}^{-\beta-\gamma}
$$

Logo,

$$
\begin{aligned}
A_{(\alpha)}^{\beta+\gamma} & =\left(A_{-\beta-\gamma+\alpha}^{-\beta-\gamma}\right)^{-1}=\left(A_{(-\gamma+\alpha)}^{-\gamma} \circ A_{(-\beta-\gamma+\alpha)}^{-\beta}\right)^{-1}=\left(A_{-\beta-\gamma+\alpha}^{-\beta}\right)^{-1} \circ\left(A_{(-\gamma+\alpha)}^{-\gamma}\right)^{-1} \\
& =A_{(-\gamma+\alpha)}^{\beta} \circ A_{(\alpha)}^{\gamma} .
\end{aligned}
$$

Resta agora considerarmos os dois casos em que $\beta$ e $\gamma$ tem sinais contrários.

Terceiro Caso: $\beta>0, \gamma \leq 0$ e $\alpha \in \mathbb{R}$.

Utilizando a definição descrita na fórmula (2.2.9) temos $A_{-\gamma+\alpha}^{\beta}=\left(A_{(-\beta-\gamma+\alpha)}^{-\beta}\right)^{-1}$. Suponha que $\beta+\gamma>0$. Novamente, temos $A_{(\alpha)}^{\beta+\gamma}=\left(A_{(-\beta-\gamma+\alpha)}^{-\beta-\gamma}\right)^{-1}$. Como $\gamma \leq 0,-\beta-\gamma \leq 0$ e $-\beta \leq 0$, o Primeiro Caso com $\widetilde{\alpha}=-\beta-\gamma+\alpha, \widetilde{\beta}=\gamma$ e $\widetilde{\gamma}=-\beta-\gamma$ implica

$$
A_{(\alpha)}^{\gamma} \circ A_{(-\beta-\gamma+\alpha)}^{-\beta-\gamma}=A_{-\beta-\gamma+\alpha}^{-\beta}
$$

Logo,

$$
A_{(\alpha)}^{\beta+\gamma}=\left(A_{(-\beta-\gamma+\alpha)}^{-\beta-\gamma}\right)^{-1}=\left(A_{(-\beta-\gamma+\alpha)}^{-\beta}\right)^{-1} \circ A_{(\alpha)}^{\gamma}=A_{(-\gamma+\alpha)}^{\beta} \circ A_{(\alpha)}^{\gamma} .
$$

Se $\beta+\gamma \leq 0$, o Primeiro Caso com $\widetilde{\alpha}=\alpha, \widetilde{\beta}=-\beta$ e $\widetilde{\gamma}=\beta+\gamma$ implica que

$$
A_{(-\beta-\gamma+\alpha)}^{-\beta} \circ A_{(\alpha)}^{\beta+\gamma}=A_{(\alpha)}^{\gamma}
$$

e obtemos a igualdade desejada.

Quarto Caso: $\beta \leq 0$ e $\gamma>0$ e $\alpha \in \mathbb{R}$.

A demonstração é análoga a do Terceiro Caso.

Sejam $\alpha, \beta>0$. Segue da Proposição 2.2.2 que a aplicação $\varphi_{\beta-\alpha,-\alpha}$ é bijetora de $X^{\beta-\alpha}$ 
em $\varphi_{\beta-\alpha,-\alpha}\left(X^{\beta-\alpha}\right)$. Defina a aplicação

$$
\widetilde{A}_{(-\alpha)}^{\beta}:=A_{(\beta-\alpha)}^{\beta} \circ \varphi_{\beta-\alpha,-\alpha}^{-1}: \varphi_{\beta-\alpha,-\alpha}\left(X^{\beta-\alpha}\right) \subseteq X^{-\alpha} \rightarrow X^{-\alpha}
$$

Denote por $\widetilde{A}_{(-\alpha)}=\widetilde{A}_{(-\alpha)}^{1}$. A aplicação $\widetilde{A}_{(-\alpha)}^{\beta}$ é bijetora, já que é composição de aplicações bijetoras. Defina

$$
\widetilde{A}_{(-\alpha)}^{-\beta}:=\left(\widetilde{A}_{(-\alpha)}^{\beta}\right)^{-1}
$$

Segue, da definição em 2.2.10, que

$$
\widetilde{A}_{(-\alpha)}^{-\beta}=\varphi_{\beta-\alpha,-\alpha} \circ A_{(-\alpha)}^{-\beta}
$$

Lema 2.2.10. Com a notação introduzida acima, $\widetilde{A}_{(-\alpha)}^{\beta}$ é uma aplicação auto-adjunta com respeito ao produto interno em $X^{-\alpha}$.

Demonstração. Primeiramente, afirmamos que:

$$
\left(R_{\alpha}^{-1} \circ \varphi_{0,-\alpha}\right)(x)=A^{-2 \alpha} x, x \in X
$$

onde $R_{\alpha}: X^{\alpha} \rightarrow X^{-\alpha}$ é o isomorfismo de Fréchet-Riesz. De fato, seja $x \in X$. O funcional linear $\varphi_{0,-\alpha}(x): X^{\alpha} \rightarrow \mathbb{R}$ é dado por $\varphi_{0,-\alpha}(x)(y)=\langle y, x\rangle_{X}$, para $y \in X^{\alpha}$. Por outro lado, como $A$ é auto-adjunto temos

$$
\langle y, x\rangle_{X}=\left\langle A^{\alpha} y, A^{\alpha} A^{-2 \alpha} x\right\rangle_{X}=\left\langle y, A^{-2 \alpha} x\right\rangle_{X^{\alpha}} .
$$

Portanto, $\varphi_{0,-\alpha} x(\cdot)=\left\langle\cdot, A^{-2 \alpha} x\right\rangle_{X^{\alpha}}$ e, desse modo, $\left(R_{\alpha} \circ A^{-2 \alpha}\right) x=\varphi_{0,-\alpha}(x)$ e 2.2.12) está demonstrada.

Fórmula 2.2.12) implica que para todo $u, v \in X$ temos

$$
\left\langle\varphi_{0,-\alpha} u, \varphi_{0,-\alpha} v\right\rangle_{X^{-\alpha}}=\left\langle A^{-2 \alpha} u, A^{-2 \alpha} v\right\rangle_{X^{\alpha}}=\left\langle A^{-\alpha} u, A^{-\alpha} v\right\rangle_{X}
$$

Como $A_{(\beta-\alpha)}^{\beta}=\left(A_{(-\alpha)}^{-\beta}\right)^{-1}$ e $A^{\beta}=\left(A^{-\beta}\right)^{-1}$, Proposição 2.2.7 implica que

$$
A_{(\beta-\alpha)}^{\beta} \circ \varphi_{\beta, \beta-\alpha}=\varphi_{0,-\alpha} \circ A^{\beta}
$$


Assim, para $u \in X^{\beta}$, temos

$$
\left(\varphi_{0,-\alpha} \circ A^{\beta}\right)(u)=\left(A_{(\beta-\alpha)}^{\beta} \circ \varphi_{\beta, \beta-\alpha}\right)(u)=\left(\widetilde{A}_{(-\alpha)}^{\beta} \circ \varphi_{\beta-\alpha,-\alpha} \circ \varphi_{\beta, \beta-\alpha}\right)(u) .
$$

Para $u, v \in X^{\beta}$, defina $x=\varphi_{\beta-\alpha,-\alpha} \varphi_{\beta, \beta-\alpha} u$ e $y=\varphi_{\beta-\alpha,-\alpha} \varphi_{\beta, \beta-\alpha} v$. É fácil ver que $y=$ $\varphi_{\beta-\alpha,-\alpha} \varphi_{\beta, \beta-\alpha} v=\varphi_{0,-\alpha} \varphi_{\beta-\alpha, 0} \varphi_{\beta, \beta-\alpha} v=\varphi_{0,-\alpha} \varphi_{\beta, 0} v$. Como $\varphi_{\beta, 0}$ é a aplicação inclusão temos

$$
\left\langle\widetilde{A}_{(-\alpha)}^{\beta} x, y\right\rangle_{X^{-\alpha}}=\left\langle A^{-\alpha} A^{\beta} u, A^{-\alpha} v\right\rangle_{X}
$$

Analogamente, obtemos

$$
\left\langle x, \widetilde{A}_{(-\alpha)}^{\beta} y\right\rangle_{X^{-\alpha}}=\left\langle A^{-\alpha} u, A^{-\alpha} A^{\beta} v\right\rangle_{X}
$$

Como $A^{\alpha} A^{\beta}=A^{\alpha+\beta}$ e $A^{\beta}$ é simétrica em $X$, temos

$$
\left\langle\widetilde{A}_{(-\alpha)}^{\beta} x, y\right\rangle_{X^{-\alpha}}=\left\langle A^{\beta} A^{-\alpha} u, A^{-\alpha} v\right\rangle_{X}=\left\langle A^{-\alpha} u, A^{\beta} A^{-\alpha} v\right\rangle_{X}=\left\langle x, \widetilde{A}_{(-\alpha)}^{\beta} y\right\rangle_{X^{-\alpha}} .
$$

Ou seja, mostramos que

$$
\left\langle\widetilde{A}_{(-\alpha)}^{\beta} x, y\right\rangle_{X^{-\alpha}}=\left\langle x, \widetilde{A}_{(-\alpha)}^{\beta} y\right\rangle_{X^{-\alpha}} \text {, para todo } x, y \in \varphi_{\beta-\alpha,-\alpha}\left(\varphi_{\beta, \beta-\alpha}\left(X^{\beta}\right)\right) \text {. }
$$

Por densidade e 2.2.16) segue que

$$
\left\langle\widetilde{A}_{(-\alpha)}^{\beta} x, y\right\rangle_{X^{-\alpha}}=\left\langle x, \widetilde{A}_{(-\alpha)}^{\beta} y\right\rangle_{X^{-\alpha}}, \text { para todo } x, y \in \varphi_{\beta, \beta-\alpha}\left(X^{\beta}\right)
$$

Isso mostra que $\widetilde{A}_{(-\alpha)}^{\beta}$ é auto-adjunta.

Segue do Lema 2.2 .10 que $\widetilde{A}_{(-\alpha)}^{-\beta}$, a aplicação invesa de $\widetilde{A}_{(-\alpha)}^{\beta}$, é simétrica em $X^{-\alpha}$.

Lema 2.2.11. Com a notação introduzida acima, para todo $\alpha, \beta, \gamma \in(0, \infty)$ temos

$$
\widetilde{A}_{(-\alpha)}^{-\beta-\gamma}=\widetilde{A}_{(-\alpha)}^{-\beta} \circ \widetilde{A}_{(-\alpha)}^{-\gamma} .
$$


Demonstração. Notemos que

$$
\begin{aligned}
& \widetilde{A}_{(-\alpha)}^{-\beta-\gamma}=\varphi_{\beta+\gamma-\alpha,-\alpha} \circ A_{(-\alpha)}^{-\beta-\gamma}, \\
& A_{(\gamma-\alpha)}^{-\beta}=\varphi_{\beta, \beta+\gamma-\alpha} \circ A^{-\beta} \circ \varphi_{0, \gamma-\alpha}^{-1} .
\end{aligned}
$$

A igualdade da Proposição 2.2 .9 implica que $A_{(-\alpha)}^{-\beta-\gamma}=A_{(\gamma-\alpha)}^{-\beta} \circ A_{(-\alpha)}^{-\gamma}$. Portanto,

$$
\widetilde{A}_{(-\alpha)}^{-\beta-\gamma}=\varphi_{\beta+\gamma-\alpha,-\alpha} \circ A_{\gamma-\alpha}^{-\beta} \circ \varphi_{\gamma-\alpha,-\alpha}^{-1} \circ \varphi_{\gamma-\alpha,-\alpha} \circ A_{(-\alpha)}^{-\gamma} .
$$

O lema está demonstrado.

Proposição 2.2.12. Seja $\alpha>0$. A aplicação $B:=\widetilde{A}_{(-\alpha)}: D(B)=\varphi_{1-\alpha,-\alpha}\left(X^{1-\alpha}\right) \subset X^{-\alpha} \rightarrow$ $X^{-\alpha}$ é auto-adjunta em $X^{-\alpha}$ e re $\sigma(B)>0$. Se $\beta>0$, seja $B^{-\beta}$ a potência fracionária básica de $B$ de ordem $-\beta$ e $X_{B}^{\beta}$ o espaço de potência fracionária correspondente. Então

$$
B^{-\beta}=\widetilde{A}_{(-\alpha)}^{-\beta} e X_{B}^{\beta}=\varphi_{\beta-\alpha,-\alpha}\left(X^{\beta-\alpha}\right)
$$

A aplicação $\varphi_{\beta-\alpha,-\alpha}$ é uma isometria entre os espaços de Hilbert $X^{\beta-\alpha}$ e $X_{B}^{\beta}$.

Demonstração. Como re $\sigma(A)>0$, existe um $\delta>0$ tal que $\langle A x, x\rangle_{X} \geq \delta\langle x, x\rangle_{X}$, para todo $x \in X$.

As igualdades 2.2.14) e 2.2.15 implicam que para $u \in X^{1}$ e $x=\varphi_{1-\alpha,-\alpha} \varphi_{1,1-\alpha} u$ temos

$$
\left\langle\widetilde{A}_{(-\alpha)}^{1} x, x\right\rangle_{X^{-\alpha}}=\left\langle A^{-\alpha} u, A^{1} A^{-\alpha} u\right\rangle_{X} \geq \delta\left\langle A^{-\alpha} u, A^{-\alpha} u\right\rangle_{X}=\delta\langle x, x\rangle_{X^{-\alpha}}
$$

Logo, pela densidade de $\varphi_{1,1-\alpha}\left(X^{1}\right)$ em $\varphi_{1-\alpha,-\alpha}\left(X^{1-\alpha}\right)=D\left(\widetilde{A}_{(-\alpha)}^{1}\right)$, obtemos $\operatorname{re} \sigma\left(\widetilde{A}_{(-\alpha)}^{1}\right)>0$. Portanto $B:=\widetilde{A}_{(-\alpha)}$ gera a família $B^{-\beta}, \beta>0$, de espaços de potências fracionárias de $B$. Portanto

$$
B^{-\beta-\gamma}=B^{-\beta} \circ B^{-\gamma}, \beta, \gamma \in(0, \infty)
$$

Afirmamos que

$$
B^{-\beta}=\widetilde{A}_{(-\alpha)}^{-\beta}, \text { para todo } \beta>0
$$

De fato, seja $Z$ o conjunto formado por $\beta \in(0, \infty)$ tal que $B^{-\beta}=\widetilde{A}_{(-\alpha)}^{-\beta}$. É fácil ver que $1 \in Z$ e usando um argumento de indução, a igualdade 2.2.17) e o Lema 2.2.11, mostramos 
que $Z$ contém todos os números inteiros.

Como um operador simétrico não-negativo definido em espaços de Hilbert possui uma única raiz quadrada não-negativa, novamente um argumento de indução (em $k \in \mathbb{N}$ ) implica que $Z$ contém todos os números da forma $m / 2^{k}$, com $m, k \in \mathbb{N}$. Defina $Z_{0}=\left\{m / 2^{k} \mid m, k \in \mathbb{N}\right\}$. $\operatorname{Logo}$ $Z_{0}$ é denso em $(0, \infty)$. Dado $\beta>0$ seja $\left(\beta_{n}\right)_{n}$ uma sequência em $Z_{0}$ tal que $\beta_{n} \rightarrow \beta$ quando $n \rightarrow \infty$.

Utilizando a igualdade (1.5.3), temos

$$
\begin{aligned}
B^{-\beta_{n}} x & =\frac{1}{\Gamma\left(\beta_{n}\right)} \int_{0}^{1} t^{\beta_{n}-1} e^{-B t} x d t, \text { para cada } n \in \mathbb{N} \text { e } x \in X^{-\alpha}, \\
B^{-\beta} x & =\frac{1}{\Gamma(\beta)} \int_{0}^{1} t^{\beta-1} e^{-B t} x d t \text { para cada } x \in X^{-\alpha}
\end{aligned}
$$

Portanto,

$$
B^{-\beta_{n}} x-B^{-\beta} x=\frac{1}{\Gamma\left(\beta_{n}\right) \Gamma(\beta)}\left[\int_{0}^{1}\left(\Gamma(\beta) t^{\beta-1}-\Gamma\left(\beta_{n}\right) t^{\beta-1}\right) e^{-B t} x d t\right]
$$

Logo, para cada $x \in X^{-\alpha}$,

$$
\left|B^{-\beta_{n}} x-B^{-\beta} x\right|_{X^{-\alpha}} \rightarrow 0, \text { quando } n \rightarrow \infty \text {. }
$$

Analogamente mostramos que para cada $x \in X$

$$
\left|A^{-\beta_{n}} x-A^{-\beta} x\right|_{X} \rightarrow 0, \text { quando } n \rightarrow \infty
$$

Seja $u \in X$. Como $\widetilde{A}_{(-\alpha)}^{-\beta_{n}} \varphi_{0,-\alpha} u=\varphi_{0,-\alpha} A^{-\beta_{n}} u$ para todo $n \in \mathbb{N}$ e $\widetilde{A}_{(-\alpha)}^{-\beta} \varphi_{0,-\alpha} u=\varphi_{0,-\alpha} A^{-\beta} u$ obtemos

$$
\left|B^{-\beta_{n}} \varphi_{0,-\alpha} u-B^{-\beta} \varphi_{0,-\alpha} u\right|_{X^{-\alpha}} \rightarrow 0, \text { quando } n \rightarrow \infty \text {. }
$$

Também $\left|A^{-\beta_{n}} u-A^{-\beta} u\right|_{X} \rightarrow 0$, quando $n \rightarrow \infty$, e desse modo $\left|\varphi_{0,-\alpha} A^{-\beta_{n}} x-\varphi_{0,-\alpha} A^{-\beta} u\right|_{X^{-\alpha}} \rightarrow$ 0 , quando $n \rightarrow \infty$. Portanto,

$$
\left|\widetilde{A}_{(-\alpha)}^{-\beta_{n}} \varphi_{0,-\alpha} u-\widetilde{A}_{(-\alpha)}^{-\beta} \varphi_{0,-\alpha} u\right|_{X^{-\alpha}} \rightarrow 0, u \in X
$$


Recordemos que $\beta_{n} \in Z_{0}$ para todo $n \in \mathbb{N}$. $\operatorname{Logo} B^{-\beta_{n}}=\widetilde{A}_{(-\alpha)}^{-\beta_{n}}$, para todo $n \in \mathbb{N}$ e segue que

$$
B^{-\beta} \varphi_{0,-\alpha} u=\widetilde{A}_{(-\alpha)}^{-\beta} \varphi_{0,-\alpha} u, \text { para todo } u \in X
$$

Como o conjunto $\varphi_{0,-\alpha}(X)$ é denso em $X^{-\alpha}$, temos $\beta \in Z$ e isto prova nossa afirmativa. A igualdade 2.2.18) implica que $X_{B}^{\beta}=\varphi_{\beta-\alpha,-\alpha}\left(X^{\beta-\alpha}\right)$.

Afirmamos agora para todo $\beta>0, \varphi_{\beta-\alpha,-\alpha}$ é uma isometria de $X^{\beta-\alpha}$ em $X_{B}^{\beta}$. De fato, $\operatorname{sejam} \tilde{x}, \tilde{y} \in X^{\beta-\alpha}$ arbitrários e defina $x=\varphi_{\beta-\alpha,-\alpha} \tilde{x}$ e $y=\varphi_{\beta-\alpha,-\alpha} \tilde{y}$.

Consideremos primeiro o caso em que existam $u, v \in X^{\beta}$ tais que $\tilde{x}=\varphi_{\beta, \beta-\alpha} u$ e $\tilde{y}=$ $\varphi_{\beta, \beta-\alpha} v$. Como $A_{(\beta-\alpha)}^{\beta} \varphi_{\beta, \beta-\alpha}=\varphi_{0,-\alpha} A^{\beta}$, temos

$$
\begin{aligned}
B^{\beta} x=\widetilde{A}_{(-\alpha)}^{\beta} x & =A_{(\beta-\alpha)}^{\beta} \varphi_{\beta-\alpha,-\alpha}^{-1} \varphi_{\beta-\alpha,-\alpha} \tilde{x} \\
& =A_{(\beta-\alpha)}^{\beta} \varphi_{\beta, \beta-\alpha} u=\varphi_{0,-\alpha} A^{\beta} u .
\end{aligned}
$$

Analogamente mostramos que $B^{\beta} y=\varphi_{0,-\alpha} A^{\beta}$. Usando 2.2.13) temos

$$
\begin{aligned}
\langle x, y\rangle_{X_{B}^{\beta}} & =\left\langle B^{\beta} x, B^{\beta} y\right\rangle_{X^{-\alpha}}=\left\langle\varphi_{0,-\alpha} A^{\beta} u, \varphi_{0,-\alpha} A^{\beta} v\right\rangle_{X^{-\alpha}} \\
& =\left\langle A^{-\alpha} A^{\beta} u, A^{-\alpha} A^{\beta} v\right\rangle_{X}=\left\langle A^{\beta-\alpha} u, A^{\beta-\alpha} v\right\rangle_{X} .
\end{aligned}
$$

Agora, se $\beta-\alpha \geq 0$, como $\varphi_{\beta, \beta-\alpha}$ é uma aplicação inclusão, temos

$$
\left\langle A^{\beta-\alpha} u, A^{\beta-\alpha} v\right\rangle_{X}=\langle u, v\rangle_{X^{\beta-\alpha}}=\langle\tilde{x}, \tilde{y}\rangle_{X^{\beta-\alpha}}
$$

Por outro lado, se $\beta-\alpha<0$, temos

$$
\begin{aligned}
\left\langle A^{\beta-\alpha} u, A^{\beta-\alpha} v\right\rangle_{X} & =\left\langle A^{-(\alpha-\beta)} u, A^{-(\alpha-\beta)} v\right\rangle_{X} \\
& =\left\langle\varphi_{0,-(\alpha-\beta)} u, \varphi_{0,-(\alpha-\beta)} v\right\rangle_{X^{-(\alpha-\beta)}}=\langle\tilde{x}, \tilde{y}\rangle_{X^{-(\alpha-\beta)}}
\end{aligned}
$$

Portanto, em ambas as situações, temos $\langle x, y\rangle_{X_{B}^{\beta}}=\langle\tilde{x}, \tilde{y}\rangle_{X^{\beta-\alpha}}$. O caso geral segue do fato do conjunto $\varphi_{\beta, \beta-\alpha}\left(X^{\beta}\right)$ ser denso em $X^{\beta-\alpha}$ e do primeiro caso. Concluímos a demonstração da proposição. 
Proposição 2.2.13. Se $\alpha \in[0,1 / 2), x \in X^{1-\alpha}$ e $v \in X^{1 / 2} \subseteq X^{\alpha}$, temos

$$
\left(A_{(1-\alpha)} x\right) \cdot v=\langle x, v\rangle_{X^{1 / 2}}
$$

onde o ponto '.' denota a função avaliação entre um elemento de $X^{-\alpha}$ e $X^{\alpha}$.

Demonstração. Suponha que $x \in X^{1}$. Segue da Proposição 2.2.7 que

$$
A_{(1-\alpha)} x=\varphi_{0,-\alpha} A x
$$

O Teorema de Frechét-Riesz e a fórmula 2.2.12) implicam que para todo $v \in X^{1 / 2}$ temos

$$
\begin{aligned}
\left(A_{(1-\alpha)} x\right) . v & =\left\langle v, R_{\alpha}^{-1}\left(A_{(1-\alpha)} x\right)\right\rangle_{\alpha}=\left\langle v, R_{\alpha}^{-1} \varphi_{0,-\alpha} A x\right\rangle_{\alpha} \\
& =\left\langle v, A^{-2 \alpha} A x\right\rangle_{\alpha}=\left\langle A^{\alpha} v, A^{\alpha} A^{-2 \alpha} A x\right\rangle_{X} \\
& =\langle v, A x\rangle_{X}=\langle v, x\rangle_{X^{1 / 2}}
\end{aligned}
$$

Portanto, o resultado está demonstrado sempre que $x \in X^{1}$. O caso geral segue da densidade de $X^{1}$ em $X^{1-\alpha}$ e em $X^{1 / 2}$.

\subsection{O problema linear abstrato}

Vamos agora analisar o problema linear abstrato associado à equação $(E R D)$.

Nesta seção vamos assumir três hipóteses básicas para desenvolver nosso trabalho.

(HL1) Sejam $a_{0}, a_{1} \in(0, \infty)$ constantes positivas e para cada $i, j=1 \ldots, N$ consideremos funções $a_{i j}: \Omega \rightarrow \mathbb{R}$, em $L^{\infty}(\Omega)$ tais que $a_{i j}=a_{j i}$ e para qualquer que seja $\xi \in \mathbb{R}^{N}$ e para quase todo $x \in \Omega$ suponha que

$$
a_{0}|\xi|^{2} \leq \sum_{i, j=1}^{N} a_{i j}(x) \xi_{i} \xi_{j} \leq a_{1}|\xi|^{2} .
$$

No que seja utilizaremos a notação $A(x):=\left(a_{i j}(x)\right)_{i, j=1}^{N}, x \in \Omega$. 
(HL2) Seja $\beta: \Omega \rightarrow \mathbb{R}$ uma função mensurável tal que para todo $\varepsilon>0$ existe uma constante $C_{\varepsilon} \geq 0$ tal que

$$
\left.\left.|| \beta\right|^{1 / 2} u\right|_{L^{2}(\Omega)} ^{2} \leq \varepsilon|u|_{H^{1}(\Omega)}^{2}+C_{\varepsilon}|u|_{L^{2}(\Omega)}^{2}, \text { para todo } u \in H_{0}^{1}(\Omega)
$$

(HL3) Suponhamos também que

$$
\lambda_{1}:=\inf \left\{\left.\int_{\Omega}\left[\sum_{i, j=1}^{N} a_{i j} \partial_{i} u \partial_{j} u+\beta|u|^{2}\right] d x\left|u \in H_{0}^{1}(\Omega),\right| u\right|_{L^{2}}=1\right\}>0 .
$$

Podemos dizer que a Hipoótese (HL3) significa que a solução do problema estacionário

$$
-\mathrm{L} u+\beta(x) u=0
$$

em $\Omega$ com potencial $\beta$ e com condições de fronteira de Dirichlet possui energia positiva.

Suponha que as Hipóteses (HL1), (HL2) e (HL3) sejam válidas. Consideremos o espaço das distribuições em $\Omega, \mathscr{D}^{\prime}(\Omega)$, e o operador L: $H_{0}^{1}(\Omega) \rightarrow \mathscr{D}^{\prime}(\Omega)$ definido por

$$
\mathrm{L} u=\sum_{i, j=1}^{N} \partial_{i}\left(a_{i j} \partial_{j} u\right), u \in H_{0}^{1}(\Omega)
$$

A definição de derivadas de distribuições implica que

$$
(\mathrm{L} u-\beta u)(v)=-\int_{\Omega}\left[\sum_{i, j=1}^{N} a_{i j} \partial_{i} u \partial_{j} v+\beta u v\right] d x, u \in H_{0}^{1}(\Omega), v \in \mathscr{D}(\Omega) .
$$

Segue por densidade, de $H_{0}^{1}(\Omega)$ em $L^{2}(\Omega)$ e de $\mathscr{D}(\Omega)$ em $H_{0}^{1}(\Omega)$, que

$$
\langle\mathrm{L} u-\beta u, v\rangle_{L^{2}(\Omega)}=-\int_{\Omega}\left[\sum_{i, j=1}^{N} a_{i j} \partial_{i} u \partial_{j} v+\beta u v\right] d x
$$

$\operatorname{para} u, v \in H_{0}^{1}(\Omega) \operatorname{com} \mathrm{L} u-\beta u \in L^{2}(\Omega)$

Lema 2.3.1. Assuma as Hipóteses (HL1), (HL2) e (HL3). Seja $\kappa \in\left[0, \lambda_{1}\right)$ arbitrário. Para cada $\bar{\varepsilon} \in\left(0, a_{0}\right)$, existe um $\rho \in(0,1)$ tal que $c:=\min \left\{\rho\left(a_{0}-\bar{\varepsilon}\right),(1-\rho)\left(\lambda_{1}-\kappa\right)-\rho\left(\bar{\varepsilon}+C_{\bar{\varepsilon}}+\kappa\right)\right\}>$ 
$0 e$

$$
\begin{aligned}
c\left(|\nabla u|_{L^{2}(\Omega)}^{2}+|u|_{L^{2}(\Omega)}^{2}\right) & \leq \int_{\Omega}\left[\sum_{i, j=1}^{N} a_{i, j} \partial_{i} u \partial_{j} u+(\beta-\kappa)|u|^{2}\right] d x \\
& \leq C\left(|\nabla u|_{L^{2}(\Omega)}^{2}+|u|_{L^{2}(\Omega)}^{2}\right), u \in H_{0}^{1}(\Omega),
\end{aligned}
$$

onde $C:=\max \left\{a_{1}+\bar{\varepsilon}, \bar{\varepsilon}+C_{\bar{\varepsilon}}\right\}$.

Demonstração. Seja $\bar{\varepsilon} \in\left(0, a_{0}\right)$. Como $a_{0}-\bar{\varepsilon}>0$ temos que

$$
0<\frac{\lambda_{1}-\kappa}{\left(\lambda_{1}-\kappa\right)+\left(\bar{\varepsilon}+\kappa+C_{\bar{\varepsilon}}\right)}<1
$$

Seja $\rho \in \mathbb{R}$ tal que

$$
0<\rho<\frac{\lambda_{1}-\kappa}{\left(\lambda_{1}-\kappa\right)+\left(\bar{\varepsilon}+\kappa+C_{\bar{\varepsilon}}\right)}<1 .
$$

Com esta escolha de $\rho \in(0,1)$ temos que

$$
(1 / \rho-1)\left(\lambda_{1}-\kappa\right)>\left(\bar{\varepsilon}+\kappa+C_{\bar{\varepsilon}}\right)
$$

e, portanto, $c>0$. Note que, a definição $\lambda_{1}$ na Hipótese (HL3) implica que qualquer que seja $u \in H_{0}^{1}(\Omega)$ temos

$$
\lambda_{1}|u|_{L^{2}(\Omega)}^{2} \leq \int_{\Omega}\left[\sum_{i, j=1}^{N} a_{i j} \partial_{i} u \partial_{j} u+\beta|u|^{2}\right] d x
$$

Logo,

$$
\begin{aligned}
& \int_{\Omega}\left[\sum_{i, j=1}^{N} \partial_{i} u \partial_{j} u+(\beta-\kappa)|u|^{2}\right] d x \geq \rho a_{0}|\nabla u|_{L^{2}}^{2}+\rho \int_{\Omega}(\beta-\kappa)|u|^{2} d x+ \\
& +(1-\rho) \lambda_{1}|u|^{2}+(1-\rho) \int_{\Omega} \kappa|u|^{2} d x \\
& =\rho a_{0}|\nabla u|_{L^{2}(\Omega)}^{2}+(1-\rho)\left(\lambda_{1}-\kappa\right)|u|_{L^{2}(\Omega)}^{2}+\rho \int_{\Omega}(\beta-\kappa)|u|^{2} d x \\
& \geq \rho a_{0}|\nabla u|_{L^{2}(\Omega)}^{2}+(1-\rho)\left(\lambda_{1}-\kappa\right)|u|_{L^{2}(\Omega)}^{2}-\rho \bar{\varepsilon}|u|_{H^{1}(\Omega)}^{2} \\
& -\rho C_{\bar{\varepsilon}}|u|_{L^{2}(\Omega)}^{2}-\rho \kappa|u|_{L^{2}(\Omega)}^{2} \\
& =\rho\left(a_{0}-\bar{\varepsilon}\right)|\nabla u|_{L^{2}(\Omega)}^{2}+(1-\rho)\left(\lambda_{1}-\kappa\right)|u|_{L^{2}(\Omega)}^{2}-\rho\left(\bar{\varepsilon}+C_{\bar{\varepsilon}}+\kappa\right)|u|_{L^{2}(\Omega)}^{2} \\
& \geq c\left(|\nabla u|_{L^{2}(\Omega)}^{2}+|u|_{L^{2}(\Omega)}^{2}\right) \text {. }
\end{aligned}
$$


Por outro lado

$$
\begin{aligned}
\int_{\Omega}\left[\sum_{i, j=1}^{N} \partial_{i} u \partial_{j} u+(\beta-\kappa)|u|^{2}\right] d x & \leq \int_{\Omega} a_{1}|\nabla u|^{2} d x+\bar{\varepsilon}|u|_{H^{1}(\Omega)}^{2}+C_{\bar{\varepsilon}}|u|_{L^{2}(\Omega)}^{2}-\kappa \int_{\Omega}|u|^{2} d x \\
& \leq a_{1}|\nabla u|_{L^{2}(\Omega)}^{2}+\bar{\varepsilon}|\nabla u|_{L^{2}(\Omega)}^{2}+\left(\bar{\varepsilon}+C_{\bar{\varepsilon}}-\kappa\right)|u|_{L^{2}(\Omega)}^{2} \\
& \leq C|\nabla u|_{L^{2}(\Omega)}^{2}+C|u|_{L^{2}(\Omega)}^{2} .
\end{aligned}
$$

E concluímos a demonstração do lema.

As estimativas feitas no Lema 2.3.1 demonstram o seguinte

Lema 2.3.2. Assuma as Hipóteses (HL1), (HL2) e (HL3). Para $u, v \in H_{0}^{1}(\Omega)$ defina

$$
\langle u, v\rangle_{1}=\int_{\Omega}\left[\sum_{i, j=1}^{N} a_{i j} \partial_{i} u \partial_{j} v+\beta u v\right] d x
$$

Então $\langle\cdot, \cdot\rangle_{1}$ é um produto interno em $H_{0}^{1}(\Omega)$ e a norma definida pelo produto interno é equivalente à norma usual em $H_{0}^{1}(\Omega)$.

Seja $D(\mathbf{A})$ o conjunto formado por todos $u \in H_{0}^{1}(\Omega)$ tais que $\operatorname{L} u-\beta u \in L^{2}(\Omega)$. Para $u \in$ $D(\mathbf{A})$ defina

$$
\mathbf{A} u=-\mathrm{L} u+\beta u
$$

Proposição 2.3.3. O operador $\mathbf{A}: D(\mathbf{A}) \rightarrow L^{2}(\Omega)$ é auto-adjunto em $X=L^{2}(\Omega)$ com re $\sigma(\mathbf{A})>$ 0 . Além disso, se $X^{\alpha}, \alpha \geq 0$, denota a família de espaços de potências fracionárias gerada por $\mathbf{A}$, então $X^{1 / 2}=H_{0}^{1}(\Omega)$ e o produto interno em $X^{1 / 2}$ é idêntico ao produto interno $\langle\cdot, \cdot\rangle_{1}$ definido no Lema 2.3.2

Demonstração. Denote o produto interno em $L^{2}(\Omega)$ por $\langle\cdot, \cdot\rangle$. Segue da fórmula (2.3.4) que

$$
\langle-\mathbf{A} u, v\rangle=\langle u,-\mathbf{A} v\rangle, u, v \in D(\mathbf{A})
$$

O Lema 2.3.1 implica que

$$
\langle-\mathbf{A} u, u\rangle \leq 0, u \in D(-\mathbf{A})
$$

Portanto, provamos que $-\mathbf{A}$ é simétrico e dissipativo. Afirmamos que o operador $-\mathbf{A}$ é $m$ dissipativo. Para isso devemos mostrar para todo $\lambda>0$ e para todo $g \in L^{2}(\Omega)$ existe um $u \in$ 
$D(-\mathbf{A})$ tal que

$$
u+\lambda \mathbf{A} u=g .
$$

De fato, defina a forma bilinear $b: H_{0}^{1}(\Omega) \times H_{0}^{1}(\Omega) \rightarrow \mathbb{R}$ por

$$
b(u, v)=\int_{\Omega} u v d x+\lambda \int_{\Omega}\left[\sum_{i, j=1}^{N} a_{i j} \partial_{i} u \partial_{j} v+\beta u v\right] d x, u, v \in H_{0}^{1}(\Omega) .
$$

As Hipóteses (HL1), (HL2) e (HL3), o Lema 2.3.1 e a Desigualdade de Schwarz implicam que existe constante $c>0$ tais que para $u, v \in H_{0}^{1}(\Omega)$

$$
|b(u, v)| \leq(1+\lambda c)|u|_{H_{0}^{1}(\Omega)}|v|_{H_{0}^{1}(\Omega)} \text { e } b(u, u) \geq c|u|_{H_{0}^{1}(\Omega)} .
$$

Uma aplicação do Teorema de Lax-Milgram implica que para cada $g \in L^{2}(\Omega)$ existe um $u \in$ $H_{0}^{1}(\Omega)$ tal que

$$
b(u, v)=\langle g, v\rangle, v \in H_{0}^{1}(\Omega) .
$$

Em particular, $u+\lambda(-\mathrm{L} u+\beta u)=g$ no sentido de distribuições e, portanto, $-\mathrm{L} u+\beta u \in L^{2}(\Omega)$. Logo $u \in D(\mathbf{A})=D(-\mathbf{A})$ e $u+\lambda \mathbf{A} u=g$. A afirmativa está demonstrada.

Agora a Proposição 1.4 .8 implica que $\mathbf{- A}$ é um operador auto-adjunto e segue do Lema 2.3.1 que re $\sigma(\mathbf{A})>0$.

Para finalizar, resta mostrarmos que $X^{1 / 2}=H_{0}^{1}(\Omega)$ e que o produto interno em $X^{1 / 2}$ tem a forma do produto interno definido no Lema 2.3.2.

Considere $\left(X,\langle\cdot, \cdot\rangle_{X}\right)=\left(L^{2}(\Omega),\langle\cdot, \cdot\rangle\right)$ e $\left(Y,\langle\cdot, \cdot\rangle_{Y}\right)=\left(H_{0}^{1}(\Omega),\langle\cdot, \cdot\rangle_{1}\right)$, onde $\langle\cdot, \cdot\rangle_{1}$ é o produto interno definido no Lema 2.3.2. Logo, $Y$ é denso em $X$ e a inclusão $j: Y \rightarrow X$ é uma aplicação contínua. Seja $B: X \rightarrow X$ o operador inverso de A. Segue que para cada $u \in X$, temos que $B u \in Y$. Ainda mais, fórmula 2.3.4 implica que para todo $v \in Y$

$$
\langle v, u\rangle_{X}=\langle v, B u\rangle_{Y}
$$

Portanto, o operador linear $B$ está nas condições do Lema 2.1.1 e o resultado segue. 


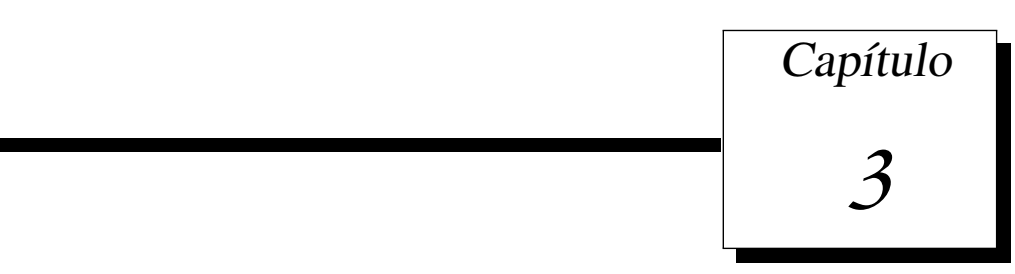

\section{O problema não-linear}

Neste capítulo apresentamos a equação de evolução abstrata associada à equação $(E R D)$. Na seção 3.1 a hipótese (HNL) é apresentada e determinamos um $\alpha \in[0,1)$ tal que de uma função $f$ satisfazendo hipótese (HNL) obtemos um operador de Nemytskiǐf : $H_{0}^{1}(\Omega) \rightarrow X^{-\alpha}$. A partir dos resultados sobre equações parabólicas apresentados no Capítulo 1, soluções de (ERD) geram um semifluxo global $\pi$ em $H_{0}^{1}(\Omega)$. O semifluxo $\pi$ é definido na Seção 3.2 .

A referência para este capítulo é o artigo [21]. Observamos que neste Capítulo $N=3$.

\subsection{Estimativas não-lineares}

Iniciamos a seção com o seguinte resultado auxiliar.

Lema 3.1.1. Sejam $X$ um espaço Banach e $A: D(A) \subset X \rightarrow X$ setorial com re $\sigma(A)>0$, gerando a família de potências fracionárias $X^{\alpha}, \alpha \geq 0$. Suponhamos que $X^{1 / 2}$ esteja continuamente imerso em $L^{6}(\Omega)$ e que $X=X^{0}$ esteja continuamente imerso em $L^{2}(\Omega)$. Então para todo $p \in[2,6)$ existe um $\bar{\beta} \in[0,1 / 2)$ tal que para todo $\alpha \in(\bar{\beta}, 1), X^{\alpha}$ está continuamente imerso em $L^{p}(\Omega)$.

Demonstração. Se $u \in X^{1 / 2}$, então $u \in L^{2}(\Omega) \cap L^{6}(\Omega)$ e segue da Interpolação para Espaços de Lebesgue que $u \in L^{p}(\Omega)$ e

$$
|u|_{L^{p}(\Omega)} \leq|u|_{L^{2}(\Omega)}^{\beta}|u|_{L^{6}(\Omega)}^{1-\beta},
$$


onde $\beta=\frac{6-p}{2 p}$. Seja $B: X^{1 / 2} \rightarrow L^{p}(\Omega)$ a aplicação inclusão de $X^{1 / 2}$ em $Y=L^{p}(\Omega)$. Segue do Teorema de Interpolação para Espaços de Potências Fracionárias (cf. Teorema 1.5.21) que existe uma constante $C \geq 0$ tal que

$$
|u|_{X^{1 / 2}} \leq C|u|_{X^{0}}^{1 / 2}|u|_{X^{1}}^{1 / 2} \text {, para todo } u \in X^{1}
$$

Logo, se $u \in D(A)=X^{1} \subset X^{1 / 2}=D(B)$ temos

$$
\begin{aligned}
|B u|_{Y} & \leq|u|_{L^{2}(\Omega)}^{\beta}|u|_{L^{6}(\Omega)}^{1-\beta} \leq C^{\prime}|u|_{X^{0}}^{\beta}|u|_{X^{1 / 2}}^{1-\beta} \\
& \leq C^{\prime}|u|_{X^{0}}^{\beta}\left(C|u|_{X^{0}}^{1 / 2}|u|_{X^{1}}^{1 / 2}\right) \leq C C^{\prime}|u|_{X^{1}}^{\beta}|u|_{X^{0}}^{1-\bar{\beta}} \\
& \leq C C^{\prime}|A u|_{X^{0}}^{\bar{\beta}}|u|_{X^{0}}^{1-\bar{\beta}},
\end{aligned}
$$

onde $\bar{\beta}=\frac{1-\beta}{2}$ e $C^{\prime}$ é uma constante positiva. Agora, segue do Lema 1.5 .24 que para todo $\alpha \in(\bar{\beta}, 1)$ a aplicação $B \circ A^{-\alpha}$ é definida e contínua de $X$ em $Y$. Logo $X^{\alpha}=R\left(A^{-\alpha}\right)$ está continuamente imerso em $L^{p}(\Omega)$.

Definição 3.1.2. Seja $w: \Omega \rightarrow \mathbb{R}$ uma função mensurável e $\gamma \in(0,1]$ um número real. Dizemos que $w \in \mathscr{E}_{\gamma}$ se, e só se, uma das condições abaixo é satisfeita:

(1) $0<\gamma<1$ e existe uma constante $c>0$ tal que para todo $\varepsilon>0$ e para todo $u \in H_{0}^{1}(\Omega)$ temos

$$
\left.\left.|| w\right|^{1 / 2} u\right|_{L^{2}(\Omega)} \leq c\left(\gamma \varepsilon|u|_{H^{1}(\Omega)}+(1-\gamma) \varepsilon^{-\gamma /(1-\gamma)}|u|_{L^{2}(\Omega)}\right)
$$

(2) $\gamma=1$ e existe uma constante $c>0$ tal que para todo $u \in H_{0}^{1}(\Omega)$

$$
\left.\left.|| w\right|^{1 / 2} u\right|_{L^{2}(\Omega)} \leq c|u|_{H^{1}(\Omega)}
$$

Pode ser mostrado a seguinte caracterização das funções em $\mathscr{E} \gamma, \gamma \in(0,1]$.

Proposição 3.1.3. Seja $w: \Omega \rightarrow \mathbb{R}$ uma função mensurável e seja $\gamma \in(0,1]$. Então $w \in \mathscr{E} \gamma$ se, e somente se, existe uma constante $C^{\prime}=C^{\prime}(w)>0$ tal que

$$
\left.\left.|| w\right|^{1 / 2} u\right|_{L^{2}(\Omega)} \leq C^{\prime}|u|_{H^{1}(\Omega)}^{\gamma}|u|_{L^{2}(\Omega)}^{1-\gamma}, \text { para todo } u \in H_{0}^{1}(\Omega)
$$

Vamos demonstrar o seguinte lema auxiliar. 
Lema 3.1.4. Suponha que $X$ seja um espaço Banach, $A: D(A) \subset X \rightarrow X$ seja um operador setorial com $\operatorname{re} \sigma(A)>0$ e $X^{\alpha}, \alpha \geq 0$, seja a família de espaços de potências fracionárias gerada por $A$. Suponhamos que $X^{1 / 2}$ esteja continuamente imerso em $H_{0}^{1}(\Omega)$ e que $X=X^{0}$ esteja continuamente imerso em $L^{2}(\Omega)$. Seja $w: \Omega \rightarrow \mathbb{R}$ e suponha que exista um $\gamma \in(0,1)$ tal que $w \in \mathscr{E} \gamma$. Então para todo $\alpha \in(\gamma / 2,1)$ a aplicação $u \mapsto|w|^{1 / 2}$ u define um operador linear e limitado de $X^{\alpha}$ em $L^{2}(\Omega)$.

Demonstração. Defina $B: X^{1 / 2} \rightarrow Y=L^{2}(\Omega)$ por $B(u)=|w|^{1 / 2} u, u \in X^{1 / 2} \subset H_{0}^{1}(\Omega)$.

O Teorema de interpolação de espaços de potências fracionárias implica que existe uma constante $C \geq 0$ tal que

$$
|u|_{X^{1 / 2}} \leq C|u|_{X^{0}}^{1 / 2}|u|_{X^{1}}^{1 / 2} \text {, para todo } x \in X^{1}
$$

Logo, existem constantes positivas $C^{\prime \prime}$ e $\widetilde{C}$ tais que para cada $u \in X^{1}=D(A) \subset X^{1 / 2}=D(B)$ temos

$$
\begin{aligned}
|B u|_{Y}=\left.\left.|| w\right|^{1 / 2} u\right|_{L^{2}(\Omega)} & \leq C^{\prime}|u|_{H^{1}(\Omega)}^{\gamma}|u|_{L^{2}(\Omega)}^{1-\gamma} \leq C^{\prime} C^{\prime \prime} \widetilde{C}|u|_{X^{1 / 2}}^{\gamma}|u|_{X^{0}}^{1-\gamma} \\
& \leq C^{\prime} C^{\prime \prime} \widetilde{C}|u|_{X^{0}}^{1-\gamma}\left(C|u|_{X^{0}}^{1 / 2}|u|_{X^{1}}^{1 / 2} \gamma^{\gamma}\right. \\
& =C^{\prime} C^{\prime \prime} \widetilde{C} C^{\gamma}|u|_{X^{1}}^{\gamma / 2}|u|_{X^{0}}^{1-\gamma / 2}=C^{\prime} C^{\prime \prime} \widetilde{C} C^{\gamma}|A u|_{X^{0}}^{\gamma / 2}|u|_{X^{0}}^{1-\gamma / 2}
\end{aligned}
$$

onde $C^{\prime}$ é a constante positiva da Proposição 3.1.3. Uma aplicação do Lema 1.5 .24 mostra que para todo $\alpha \in(\gamma / 2,1)$ a aplicação $B \circ A^{-\alpha}$ é definida e limitada de $X$ em $Y$. Portanto, $B$ é uma aplicação linear e limitada de $X^{\alpha}=R\left(A^{-\alpha}\right)$ em $Y=L^{2}(\Omega)$, como desejado.

Nesta seção, consideremos a seguinte hipótese:

\section{HiPÓTESE (HNL):}

(a) $\bar{c} \geq 0$ e $\bar{\rho} \geq 0$ são constantes com $\bar{\rho} \in[2,4)$ e $c: \Omega \rightarrow[0, \infty)$ é uma função em $L^{1}(\Omega)$;

(b) $a: \Omega \rightarrow \mathbb{R}$ é uma função mensurável tal que $a \in \mathscr{E} \gamma$, para algum $\gamma \in(0,1)$;

(c) $f: \Omega \times \mathbb{R} \rightarrow \mathbb{R}$ é uma função tal que $\Omega \ni x \mapsto f(x, u)$ é uma função Lebesgue-mensurável para todo $u \in \mathbb{R}$ e $\mathbb{R} \ni u \mapsto f(x, u)$ é uma função de classe $C^{1}$ para q.t.p. $x \in \Omega$; 
(d) $\left|\partial_{u} f(x, u)\right| \leq \bar{c}\left(a(x)+|u|^{\bar{\rho}}\right)$, para todo $u \in \mathbb{R}$ e q.t.p. $x \in \Omega$;

(e) $f(\cdot, 0) \in L^{2}(\Omega)$;

(f) $f(x, u) u \leq c(x)$ e $\int_{0}^{u} f(x, s) d s \leq c(x)$ para q.t.p. $x \in \Omega$ e todo $u \in \mathbb{R}$.

No restante desta seção vamos considerar $X=L^{2}(\Omega), A: D(A) \subset X \rightarrow X$ um operador setorial com re $\sigma(A)>0$, gerando a família de espaços de potências fracionárias $X^{\alpha}, \alpha \geq 0$.

Suponhamos que $X^{1 / 2}$ esteja continuamente imerso em $H_{0}^{1}(\Omega)$. Seja $f: \Omega \times \mathbb{R} \rightarrow \mathbb{R}$ uma função satisfazendo Hipótese (HNL). Sejam $q=(6 /(\bar{\rho}+1))$ e $p=(q /(q-1))$.

Se $\bar{\rho}>2$ ou $a^{2} \notin \mathscr{E} 1$, então escolhemos $\alpha \in(0,1 / 2)$ tal que

$$
\alpha>\max \left\{\gamma / 2, \frac{1-(6-p) / 2 p}{2}\right\}
$$

O Lema 3.1.1 implica que $X^{\alpha}$ está continuamente imerso em $L^{p}(\Omega)$ e segue do Lema 3.1.4 que a aplicação $u \mapsto|a|^{1 / 2} u$ é limitada de $X^{\alpha}$ em $X$.

Se $\bar{\rho}=2$ e $a^{2} \in \mathscr{E} 1$, escolhemos $\alpha=0$.

Seja $F: \Omega \times \mathbb{R} \rightarrow \mathbb{R}$ a função dada por

$$
F(x, u)=\int_{0}^{u} f(x, s) d s
$$

sempre que $s \mapsto f(x, s)$ for contínua e $F(x, u)=0$, caso contrário.

Proposição 3.1.5. Com a notação introduzida acima, para todo $u \in X^{1 / 2}, \widehat{F}(u)$, o operador de Nemytskǐ̌ $\widehat{F}$ em u, está em $L^{1}(\Omega)$. Além disso $\widehat{F}: X^{1 / 2} \rightarrow L^{1}(\Omega)$ é um operador de NemytskiǔFréchet diferenciável com

$$
D \widehat{F}(u) \cdot h=\widehat{f}(u) \cdot h, \text { para todo } u, h \in X^{1 / 2}
$$

Demonstração. Notemos que se $u \in X^{1 / 2}$, então $u \in L^{2}(\Omega) \cap L^{6}(\Omega)$ e, portanto, $u \in L^{r}(\Omega)$, para todo $r \in[2,6]$. Em particular, as aplicações $\widehat{f}(u): \Omega \rightarrow \mathbb{R}$ e $\widehat{F}(u): \Omega \rightarrow \mathbb{R}$ são mensuráveis. 
Mostremos que $\widehat{F}(u) \in L^{1}(\Omega)$ para todo $u \in X^{1 / 2}$. De fato, seja $u \in X^{1 / 2}$, temos

$$
\begin{aligned}
\int_{\Omega}|\widehat{F}(u)(x)| d x & =\int_{\Omega}|F(x, u(x))| d x \leq \int_{\Omega} \int_{0}^{u(x)}|f(x, s)| d s d x \\
& \leq \int_{\Omega}\left[\int_{0}^{u(x)}|f(x, s)-f(x, 0)| d s+\int_{0}^{u(x)}|f(x, 0)| d s\right] d x .
\end{aligned}
$$

Temos que

$$
f(x, s)-f(x, 0)=\int_{0}^{1} \frac{d}{d t} f(x, s t) d t=\int_{0}^{1} \frac{\partial}{\partial u} f(x, s t) s d t .
$$

Como $\partial_{u} f$ é contínua para q.t.p. $x \in \Omega$, o Teorema do Valor Médio implica que

$$
|f(x, s)-f(x, 0)| \leq \max _{\xi \in[0, s]}\left|\partial_{u} f(x, \xi)\right||s|
$$

Segue da Hipótese (HNL) que

$$
\left|\partial_{u} f(x, \xi)\right| \leq \bar{C}\left(|a(x)|+|\xi|^{\bar{\rho}}\right), \text { para todo } \xi \in \mathbb{R} \text { e q.t.p. } x \in \Omega \text {. }
$$

Utilizando desigualdade 3.1.4 em 3.1.3 obtemos

$$
|f(x, s)-f(x, 0)| \leq \bar{C}\left(|a(x)|+|s|^{\bar{\rho}}\right)|s| \text {, para q.t.p } x \in \Omega \text {. }
$$

Logo,

$$
\begin{aligned}
\int_{\Omega} \int_{0}^{u(x)} \int_{0}^{1}\left|\partial_{u} f(x, s t)\right||s| d t d s d x & \leq \bar{C} \int_{\Omega} \int_{0}^{u(x)} \int_{0}^{1}\left(|a(x)|+|t s|^{\bar{\rho}}\right)|s| d t d s d x \\
& \leq \bar{C} \int_{\Omega} \int_{0}^{u(x)}\left(|a(x)||s|+|s|^{\bar{\rho}+1}\right) d s d x \\
& \leq \bar{C} \int_{\Omega}\left(|a(x)| \frac{|u(x)|^{2}}{2}+|u(x)|^{\mid \bar{\rho}+2}\right) d x
\end{aligned}
$$

Com isso,

$$
\begin{aligned}
\int_{\Omega}|\widehat{F}(u)(x)| d x & \leq \int_{\Omega}|f(x, 0) u(x)| d x+\int_{\Omega} \int_{0}^{u(x)} \int_{0}^{1}\left|\partial_{u} f(x, s t)\right||s| d t d s d x \\
& \leq \int_{\Omega}|f(x, 0) u(x)| d x+\bar{C} \int_{\Omega}\left(\left.\left.\left.|| a(x)\right|^{1 / 2} u(x)\right|^{2}|+| u(x)\right|^{\bar{\rho}+2}\right) d x<\infty
\end{aligned}
$$

e isso mostra que $\widehat{F}(u) \in L^{1}(\Omega)$ sempre que $u \in X^{1 / 2}$. 
Afirmamos que para cada $u \in X^{1 / 2}$, a aplicação $X^{1 / 2} \ni h \mapsto \widehat{f}(u) \cdot h \in L^{1}(\Omega)$ é linear e limitada. A prova da linearidade da aplicação é imediata. Sejam $u, h \in X^{1 / 2}$. Utilizando a desigualdade 3.1 .5 temos

$$
\begin{aligned}
\int_{\Omega}|\widehat{f}(u) \cdot h| d x & =\int_{\Omega}|f(x, u(x)) h(x)| d x \\
& \leq \int_{\Omega}|f(x, 0) h(x)| d x+\int_{\Omega}|f(x, u(x))-f(x, 0)||h(x)| d x \\
& \leq|\widehat{f}(0)|_{L^{2}(\Omega)}|h|_{L^{2}(\Omega)}+\bar{C} \int_{\Omega}|a(x) u(x) h(x)| d x+\bar{C} \int_{\Omega}|u(x)|^{\bar{\rho}}|u(x) h(x)| d x \\
& \leq|\widehat{f}(0)|_{L^{2}(\Omega)}|h|_{L^{2}(\Omega)}+\left.\left.\left.\left.\bar{C}|| a\right|^{1 / 2} u\right|_{L^{2}(\Omega)}|| a\right|^{1 / 2} h\right|_{L^{2}(\Omega)}+\bar{C}|u|_{L^{r}(\Omega)}^{\bar{\rho}+1}|h|_{L^{6}(\Omega)},
\end{aligned}
$$

onde $r=(\bar{\rho}+1) 6 / 5$. Portanto, concluímos que existe uma constante $\widetilde{M}_{u} \geq 0$ tal que

$$
|\widehat{f}(u) \cdot h|_{L^{1}(\Omega)} \leq \widetilde{M}_{u}|h|_{X^{1 / 2}}, \text { para todo } h \in X^{1 / 2}
$$

e isso significa que a aplicação $h \mapsto \widehat{f}(u) \cdot h$ é linear e limitada para cada $u \in X^{1 / 2}$.

Mostraremos que o operador de Nemytskiř $\widehat{F}: X^{1 / 2} \rightarrow L^{1}(\Omega)$ é Fréchet diferenciável e que $D \widehat{F}(u) . h=\widehat{f}(u) . h$, para todo $u, h \in X^{1 / 2}$. De fato, sejam $u, h \in X^{1 / 2}$. Notemos que

$$
\begin{aligned}
\mid \widehat{F}(u+h)-\widehat{F}(u) & -\left.\widehat{f}(u) \cdot h\right|_{L^{1}(\Omega)} \\
& =\int_{\Omega}|F(x, u(x)+h(x))-F(x, u(x))-f(x, u(x)) h(x)| d x .
\end{aligned}
$$

\section{Como}

$$
\begin{aligned}
F(x, u(x)+h(x))-F(x, u(x)) & =\int_{0}^{1} \frac{d}{d t} F(x, u(x)+\operatorname{th}(x)) d t \\
& =\int_{0}^{1} \frac{\partial}{\partial u} F(x, u(x)+\operatorname{th}(x)) h(x) d t \\
& =\int_{0}^{1} \frac{\partial}{\partial u}\left(\int_{0}^{u(x)+\operatorname{th}(x)} f(x, s) d s\right) h(x) d t \\
& =\int_{0}^{1} f(x, u(x)+\operatorname{th}(x)) h(x) d t
\end{aligned}
$$


segue que

$$
\begin{aligned}
\mid \widehat{F}(u+h)-\widehat{F}(u) & -\left.\widehat{f}(u) h\right|_{L^{1}(\Omega)} \\
& \leq \int_{\Omega} \int_{0}^{1}|f(x, u(x)+\operatorname{th}(x))-f(x, u(x))||h(x)| d t d x .
\end{aligned}
$$

Com um cálculo semelhante ao anterior obtemos

$$
|f(x, u(x)+\operatorname{th}(x))-f(x, u(x))| \leq \bar{C} t|h(x)|\left[|a(x)|+2^{\bar{\rho}-1}\left(|u(x)|^{\bar{\rho}}+(t|h(x)|)^{\bar{\rho}}\right)\right]
$$

e substituindo em (3.1.6) obtemos

$$
\begin{aligned}
\mid \widehat{F}(u+h)-\widehat{F}(u) & -\left.\widehat{f}(u) h\right|_{L^{1}(\Omega)} \leq \int_{\Omega} \int_{0}^{1} \bar{C}|h(x)|^{2}|a(x)| t d t d x+ \\
& +\int_{\Omega} \int_{0}^{1}\left[2^{\bar{\rho}-1}\left(|u(x)|^{\bar{\rho}} t+t^{\bar{\rho}+1}|h(x)|^{\bar{\rho}}\right)\right] d t d x \\
& \leq\left.\int_{\Omega} \bar{C} \frac{\overline{2}}{2}|a(x)|^{1 / 2} h(x)\right|^{2} d x+ \\
& +\int_{\Omega} \bar{C}|h(x)|^{2} 2^{\bar{\rho}-1}\left(|u(x)|^{\bar{\rho}}+|h(x)|^{\bar{\rho}}\right) d x \\
& \leq\left.\left.\bar{C}|| a\right|^{1 / 2} h\right|_{L^{2}(\Omega)} ^{2}+\bar{C} 2^{\bar{\rho}-1}\left(|u|_{L^{r}(\Omega)}^{\bar{\rho}}+|h|_{L^{r}(\Omega)}^{\bar{\rho}}\right)|h|_{L^{6}(\Omega)}^{2},
\end{aligned}
$$

onde $r=(6 \bar{\rho}) / 4$. Ou seja, mostramos que existem constantes $M, K \geq 0$ tais que

$$
|\widehat{F}(u+h)-\widehat{F}(u)-\widehat{f}(u) h|_{L^{1}(\Omega)} \leq \bar{C}\left[K+2^{\bar{\rho}-1} M\left(|u|_{L^{r}(\Omega)}^{\bar{\rho}}+|h|_{L^{r}(\Omega)}^{\bar{\rho}}\right)\right]|h|_{X^{1 / 2}}^{2}
$$

A demonstração da proposição está completa.

Se $\bar{\rho}>2$ ou $a^{2} \notin \mathscr{E} 1$, para cada $u \in X^{1 / 2}$, considere a função $\mathbf{f}(u): X^{\alpha} \rightarrow \mathbb{R}$ definida por

$$
v \mapsto \int_{\Omega} f(x, u(x)) v(x) d x
$$

Se $\bar{\rho}=2$ e $a^{2} \in \mathscr{E}_{1}$, para cada $u \in X^{1 / 2}$, defina $\mathbf{f}(u):=\widehat{f}(u) \in X=X^{-\alpha}$.

Proposição 3.1.6. Suponha a notação desta seção. Se $\bar{\rho}>2$ ou $a^{2} \notin \mathscr{E} 1$, para todo $u \in X^{1 / 2}$, $\mathbf{f}(u) \in X^{-\alpha}$. Além disso, em ambos os casos definidos acima, a aplicação $\mathbf{f}: X^{1 / 2} \rightarrow X^{-\alpha} e^{\prime}$ Lipschitziana em subconjuntos limitados de $X^{1 / 2}$.

Demonstração. Considere o caso $\bar{\rho}>2$ ou $a^{2} \notin \mathscr{E}_{1}$. Seja $u \in X^{1 / 2}$. Como $X^{\alpha}$ está continua- 
mente imerso em $L^{2}(\Omega)$ e em $L^{p}(\Omega)$, para todo $v \in X^{\alpha}$ temos

$$
\begin{aligned}
\int_{\Omega}|f(x, u(x)) v(x)| d x & \leq \int_{\Omega}[|f(x, 0) v(x)|+|f(x, u(x))-f(x, 0)||v(x)|] d x \\
& \leq|\widehat{f}(0)|_{L^{2}(\Omega)}|v|_{L^{2}(\Omega)}+\int_{\Omega} \bar{C}|a(x) u(x) v(x)|+\bar{C}|u(x)|^{\bar{\rho}+1}|v(x)| d x \\
& \leq|\widehat{f}(0)|_{L^{2}(\Omega)}|v|_{L^{2}(\Omega)}+\left.\left.\bar{C}|| a\right|^{1 / 2} u\right|_{L^{2}(\Omega)}+\bar{C}|u|_{L^{6}(\Omega)}^{\bar{\rho}+1}|v|_{L^{p}(\Omega)} \\
& \leq \mathrm{C}\left(|\widehat{f}(0)|_{L^{2}(\Omega)}+\left.\left.\overline{C K}|| a\right|^{1 / 2} u\right|_{L^{2}(\Omega)}+\bar{C}|u|_{L^{6}(\Omega)}^{\bar{\rho}+1}\right)|v|_{X^{\alpha}}
\end{aligned}
$$

onde C, $\bar{K}$ são constantes em $[0, \infty)$. Mostramos que a função $\mathbf{f}(u): X^{\alpha} \rightarrow \mathbb{R}$, dada por $\mathbf{f}(u)(v)=$ $\int_{\Omega} f(x, u(x)) v(x) d x, v \in X^{\alpha}$, está bem definida, é linear e limitada, ou seja, $\mathbf{f}(u) \in X^{-\alpha}$.

Analogamente, se $u, h \in X^{1 / 2}$ e $v \in X^{\alpha}$, obtemos

$$
\begin{aligned}
\int_{\Omega} \mid(f(x, u(x)+h(x)) & -f(x, u(x))) v(x)\left|d x \leq \bar{C} \int_{\Omega}(|a(x) h(x) v(x)|)\right| v(x) \mid d x \\
& +\int_{\Omega} 2^{\bar{\rho}-1}\left(\left(|u(x)|^{\bar{\rho}}+|h(x)|^{\bar{\rho}}\right)|h(x)|\right)|v(x)| d x \\
& \leq \bar{C}\left(\left.\left.|| a\right|^{1 / 2} h\right|_{L^{2}(\Omega)}+2^{\bar{\rho}-1}\left(|u|_{L^{6}(\Omega)}^{\bar{\rho}}+|h|_{L^{6}(\Omega)}^{\bar{\rho}}\right)|h|_{L^{6}(\Omega)}\right)|v|_{L^{p}(\Omega)} \\
& \leq \mathrm{C}\left(\left.\left.\bar{C} K|| a\right|^{1 / 2} h\right|_{L^{2}}+2^{\bar{\rho}-1} \bar{C}\left(|u|_{L^{6}(\Omega)}^{\bar{\rho}}+|h|_{L^{6}(\Omega)}^{\bar{\rho}}\right)|h|_{L^{6}(\Omega)}\right)|v|_{X^{\alpha}}
\end{aligned}
$$

o que demonstra que a aplicação $\mathbf{f}: X^{1 / 2} \rightarrow X^{-\alpha}$ está bem definida e é Lipschitziana em subconjuntos limitados de $X^{1 / 2}$.

Para concluir a demonstração, se $\bar{\rho}=2$ e $a^{2} \in \mathscr{E} 1$, com cálculos análogos mostramos que as desgiualdades

$$
|\widehat{f}(u)|_{L^{2}(\Omega)} \leq|\widehat{f}(0)|_{L^{2}(\Omega)}+\bar{C}\left(|a u|_{L^{2}(\Omega)}+|u|_{L^{2(\bar{\rho}+1)}(\Omega)}^{\bar{\rho}+1}\right)
$$

e

$$
|\widehat{f}(u+h)-\widehat{f}(u)|_{L^{2}(\Omega)} \leq \bar{C}|a h|_{L^{2}(\Omega)}+2 \bar{C}\left(|u|_{L^{2(\bar{\rho}+1)}(\Omega)}^{\bar{\rho}}+|h|_{L^{2(\bar{\rho}+1)}(\Omega)}^{\bar{\rho}}\right)|h|_{L^{2(\bar{\rho}+1)}(\Omega)}
$$

são verdadeiras. Disso segue que o operador $\mathbf{f}: X^{1 / 2} \rightarrow X^{-\alpha}=X$ é bem definido e Lipschitz em limitados de $X^{1 / 2}$. 


\subsection{A equação de evolução abstrata}

Neste seção vamos assumir as Hipóteses (HL1), (HL2), (HL3) e (HNL). Sejam A o operador linear definido na Seção $2.3 \mathrm{e} X^{\alpha}, \alpha \in \mathbb{R}$, a família de espaços de potências fracionárias gerados por A. Sejam $\widetilde{\mathbf{A}}_{(\alpha)}, \alpha \in \mathbb{R}$ e $-\widetilde{\mathbf{A}}_{(-\alpha)}, \alpha \in(0, \infty)$ os operadores definidos na Seção 2.2 , onde $A=\mathbf{A}$. Seja $f: \Omega \times \mathbb{R} \rightarrow \mathbb{R}$ uma função satisfazendo Hipótese (HNL).

Se $\bar{\rho}=2$ e $a^{2} \in \mathscr{E} 1$, então a equação parabólica

$$
\dot{u}=-\mathbf{A} u+\mathbf{f}(u)
$$

define um semifluxo local $\pi$ em $X^{1 / 2}$ e para cada $u \in X^{1 / 2}, t \in\left[0, \omega_{u}\right), u \pi t \in X$.

Se $\bar{\rho} \in(2,4)$ ou $a^{2} \notin \mathscr{E}_{1}$, escolha um $\alpha \in(0,1 / 2)$ como em 3.1 .1$)$.

Notemos que segue da Proposição 2.2.12 que o operador $\widetilde{\mathbf{A}}_{(-\alpha)}$ é auto-adjunto com $\operatorname{re} \sigma\left(\widetilde{\mathbf{A}}_{(-\alpha)}\right)>0$. Além disso, os espaços de potências fracionárias gerados por $\mathbf{A}$ e $\widetilde{\mathbf{A}}_{(-\alpha)}$ são isometricamente isomorfos. Com isso a equação parabólica

$$
\dot{\tilde{u}}=-\widetilde{\mathbf{A}}_{(-\alpha)} \tilde{u}+\mathbf{f}\left(\varphi_{1 / 2,-\alpha}^{-1} \tilde{u}\right)
$$

define um semifluxo local $\tilde{\pi}$ em $\varphi_{1 / 2,-\alpha}\left(X^{1 / 2}\right)$.

Para este caso, seja $\pi$ o semifluxo local em $X^{1 / 2}$ definido como o semifluxo conjugado a $\tilde{\pi}$ pela aplicação $\varphi_{1 / 2,-\alpha}: X^{1 / 2} \rightarrow \varphi_{1 / 2,-\alpha}\left(X^{1 / 2}\right)$.

É claro que o semifluxo local $\tilde{\pi}$ depende de $\alpha$. O próximo resultado mostra que isso não ocorre com o semifluxo local $\pi$.

Proposição 3.2.1. Suponha que $\bar{\rho} \in(2,4)$ ou $a^{2} \notin \mathscr{E}_{1}$ e seja $\alpha \in(0,1 / 2)$ como em 3.1.1). $O$ semifluxo $\pi$ em $X^{1 / 2}$, definido como o semifluxo conjugado a $\tilde{\pi}$ pela aplicação $\varphi_{1 / 2,-\alpha}: X^{1 / 2} \rightarrow$ $\varphi_{1 / 2,-\alpha}\left[X^{1 / 2}\right]$, independe da escolha de $\alpha$.

Demonstração. Sejam $\alpha, \beta \in(0,1 / 2)$ como em 3.1.1). Note que $X^{1 / 2}$ é isometricamente ismorfo a $\varphi_{1 / 2,-\alpha}\left(X^{1 / 2}\right)$ e a $\varphi_{1 / 2,-\beta}\left(X^{1 / 2}\right)$ por meio de $\varphi_{1 / 2,-\alpha}$ e $\varphi_{1 / 2,-\beta}$ respectivamente. Logo, os espaços $X_{\alpha}:=\varphi_{1 / 2,-\alpha}\left(X^{1 / 2}\right)$ e $X_{\beta}:=\varphi_{1 / 2,-\beta}\left(X^{1 / 2}\right)$ são isometricamente isomorfos pela aplicação $\varphi_{1 / 2,-\beta} \circ \varphi_{1 / 2,-\alpha}^{-1}=\varphi_{-\alpha,-\beta}: X_{\alpha} \rightarrow X_{\beta}$. 
Consideremos $\tilde{\pi}_{\alpha}$ e $\tilde{\pi}_{\beta}$ os semifluxos gerados pelas soluções das equações parabólicas

$$
\begin{aligned}
\dot{\tilde{u}} & =-\widetilde{\mathbf{A}}_{(-\alpha)} \widetilde{u}+\mathbf{f}_{\alpha}\left(\varphi_{1 / 2,-\alpha}^{-1} \widetilde{u}\right), \\
\dot{\tilde{v}} & =-\widetilde{\mathbf{A}}_{(-\beta)} \widetilde{v}+\mathbf{f}_{\beta}\left(\varphi_{1 / 2,-\alpha}^{-1} \widetilde{v}\right),
\end{aligned}
$$

respectivamente. Seja $\widehat{\pi}_{\beta}$ o semifluxo local definido em $X_{\beta}$ o qual é conjugado ao semifluxo $\widetilde{\pi}_{\alpha}$ pela aplicação $\varphi_{-\alpha,-\beta}: X_{\alpha} \rightarrow X_{\beta}$. Logo, para $v \in X_{\beta}$,

$$
v \widehat{\pi}_{\beta} t=\varphi_{-\alpha,-\beta}\left(\varphi_{-\alpha,-\beta}^{-1}(v) \tilde{\pi}_{\alpha} t\right), \text { para todo } t \in\left[0, \omega_{\widehat{\pi}_{\beta}, v}\right)
$$

A Fórmula da Variação das Constantes do Lema 1.7 .2 implica que para $t \in\left[0, \omega_{\widehat{\pi}_{\beta}, v}\right)$

$$
\begin{aligned}
& v \widehat{\pi}_{\beta} t=\varphi_{-\alpha,-\beta}\left(e^{-\widetilde{\mathbf{A}}_{(-\alpha)} t} \varphi_{-\alpha,-\beta}^{-1} \nu+\int_{0}^{t} e^{-\widetilde{\mathbf{A}}_{(-\alpha)}(t-s)} \mathbf{f}_{\alpha}\left(\varphi_{1 / 2,-\alpha}^{-1}\left[\varphi_{-\alpha,-\beta}^{-1} \nu \widetilde{\pi}_{\alpha} s\right]\right) d s\right) \\
& =\varphi_{-\alpha,-\beta}\left(\varphi_{-\alpha,-\beta}^{-1} e^{-\widetilde{\mathbf{A}}_{(-\beta)}^{t}} v\right)+ \\
& +\varphi_{-\alpha,-\beta}\left(\int_{0}^{t} \varphi_{-\alpha,-\beta}^{-1} e^{-\widetilde{\mathbf{A}}_{(-\beta)}(t-s)} \varphi_{-\alpha,-\beta} \mathbf{f}_{\alpha}\left(\varphi_{1 / 2,-\beta}^{-1} \circ \varphi_{-\alpha,-\beta}\left[\varphi_{-\alpha,-\beta}^{-1} \nu \widetilde{\pi}_{\alpha} s\right]\right) d s\right) \\
& =e^{-\widetilde{\mathbf{A}}_{(-\beta)}{ }^{t}} v+\int_{0}^{t} e^{-\widetilde{\mathbf{A}}_{(-\beta)}(t-s)} \mathbf{f}_{\beta}\left(\varphi_{1 / 2,-\beta}^{-1} v \widehat{\pi}_{\beta} s\right) d s .
\end{aligned}
$$

Portanto, segue da unicidade da solução para equações parabólicas que $t \in\left[0, \omega_{\widetilde{\pi}_{\beta}}\right)$ e $v \widehat{\pi}_{\beta} t=$ $v \widetilde{\pi}_{\beta} t$. Analogamente, se $t \in\left[0, \omega_{\widetilde{\pi}_{\beta}, v}\right)$ temos $t \in\left[0, \omega_{\widehat{\pi}_{\beta}, v}\right)$ e $u \widetilde{\pi}_{\beta} t=u \widehat{\pi}_{\beta} t$. Ou seja, os semifluxos $\tilde{\pi}_{\alpha}$ e $\tilde{\pi}_{\beta}$ são conjugados pela aplicação $\varphi_{-\alpha,-\beta}$.

Consideremos, agora, $\pi_{\alpha}$ e $\pi_{\beta}$ os semifluxos locais definidos em $X^{1 / 2}$ os quais conjugados com $\tilde{\pi}_{\alpha}$ e $\tilde{\pi}_{\beta}$, respectivamente. Para $u \in X^{1 / 2}$ e para cada $t \in\left[0, \omega_{\pi_{\beta}, u}\right)$ temos

$$
\begin{aligned}
u \pi_{\beta} t=\varphi_{1 / 2,-\beta}^{-1}\left(\varphi_{1 / 2,-\beta} u \tilde{\pi}_{\beta} t\right) & =\varphi_{1 / 2,-\beta}^{-1}\left(\varphi_{-\alpha,-\beta}\left(\varphi_{-\alpha,-\beta}^{-1} \varphi_{1 / 2,-\beta} u\right) \widetilde{\pi}_{\alpha} t\right) \\
& =\varphi_{1 / 2,-\beta}^{-1} \varphi_{-\alpha,-\beta}\left(\varphi_{1 / 2,-\alpha} u \widetilde{\pi}_{\alpha} t\right) \\
& =\varphi_{1 / 2,-\alpha}^{-1}\left(\varphi_{1 / 2,-\alpha} u \widetilde{\pi}_{\alpha} t\right) .
\end{aligned}
$$

Logo, $t \in\left[0, \omega_{\pi_{\alpha}, u}\right)$ e $u \pi_{\beta} t=u \pi_{\alpha} t$. Analogamente, se $t \in\left[0, \omega_{\pi_{\alpha}, u}\right)$ temos $t \in\left[0, \omega_{\pi_{\beta}, u}\right)$ e $u \pi_{\beta} t=u \pi_{\alpha} t$. Com isso demonstramos está completa.

Escolhendo $\alpha=0$ se $\bar{\rho}=2$ e $a^{2} \in \mathscr{E}_{1}$, o seguinte resultado segue da definição de $\pi$ : 
Proposição 3.2.2. Sejam $T \in(0, \infty), u:[0, T) \rightarrow X^{1 / 2}$ uma solução de $\pi$. Então u é contínua em $[0, T)$, diferenciável $\left(e m X^{1 / 2}\right)$ em $(0, T)$ e, para $t \in(0, T)$, temos

$$
\varphi_{0,-\alpha}(\dot{u}(t))=-\mathbf{A}_{(1-\alpha)} u(t)+\mathbf{f}(u(t))
$$

onde $\dot{u}(t):=\partial\left(u ; X^{1 / 2}\right)(t)=\partial\left(u ; X^{0}\right)(t)$.

Demonstração. A definição de solução implica que a função $u$ é contínua e diferenciável. Como $X^{1 / 2}$ está continuamente imerso em $X^{0}$, então $\partial\left(u ; X^{1 / 2}\right)(t)=\partial\left(u ; X^{0}\right)(t)$, onde $\partial\left(u ; X^{\beta}\right)(t)$ denota a derivada parcial de $u \mathrm{em} X^{\beta}$ no instante $t$, para qualquer $\beta$. Portanto, devemos demonstrar apenas a igualdade 3.2 .3 .

Note que $\mathbf{A}_{(1-\alpha)}=\varphi_{0,-\alpha} \circ \mathbf{A} \circ \varphi_{1,1-\alpha}^{-1}$. Como $\alpha \in(0,1 / 2)$, se $u_{0}:=u(0) \in X^{1 / 2}$, temos

$$
u(t)=u_{0} \pi t=\varphi_{1 / 2,-\alpha}^{-1}\left(\varphi_{1 / 2,-\alpha} u_{0} \tilde{\pi} t\right), \text { para } t \in(0, T) .
$$

Logo,

$$
\varphi_{1 / 2,-\alpha} \dot{u}(t)=-\widetilde{\mathbf{A}}_{(-\alpha)} \varphi_{1 / 2,-\alpha} u(t)+\mathbf{f}\left(\varphi_{1 / 2,-\alpha}^{-1} \varphi_{1 / 2,-\alpha} u(t)\right)
$$

e, portanto

$$
\varphi_{0,-\alpha} \varphi_{1 / 2,0} \dot{u}(t)=-\mathbf{A}_{(1-\alpha)} \varphi_{1-\alpha,-\alpha}^{-1} \varphi_{1 / 2,-\alpha} u(t)+\mathbf{f}(u(t))
$$

e obtemos a igualdade 3.2 .3 . 


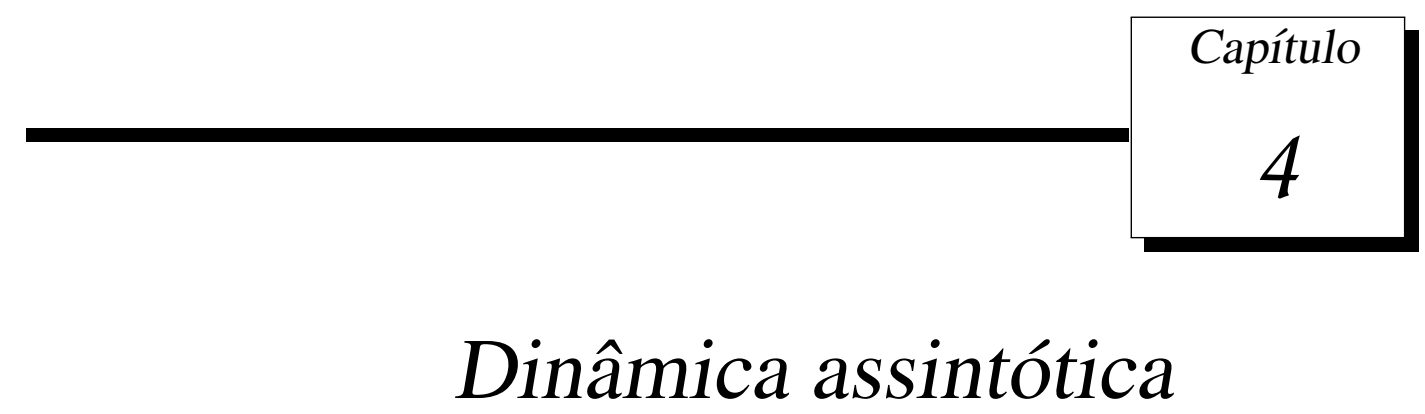

Finalmente neste capítulo demonstramos o principal resultado deste trabalho: assumindo as hipóteses (HL1), (HL2), (HL3) e (HNL) o semifluxo $\pi$ possui um atrator global (ver Teorema 4.4.2. .

Na seção 4.2 mostramos que $\pi$ (definido na Seção 3.2 ) é um semifluxo gradiente, isto é construimos uma função de Lyapunov associada a $\pi$.

Na seção 4.3 apresentamos a ferramenta do trucamento inspirada pelo trabalho [24] que os autores Prizzi e Rybakowski utilizaram em [21] para concluir que o semifluxo global $\pi$ é assintoticamente compacto.

Iniciamos o capítulo com um resultado geral de perturbação de soluções para equações parabólicas semilineares e um resultado geral sobre regularidade de conjuntos invariantes em espaços de Hilbert.

A referência básica para este capítulo é o artigo [21].

\subsection{Equações parabólicas semilineares}

Suponha que $X$ seja um espaço de Banach e $A: D(A) \subset X \rightarrow X$ seja um operador setorial. Seja $\bar{k} \in[0, \infty)$ uma constante tal que re $(A+\bar{k} I)>0$ e seja $X^{\beta}, \beta \geq 0$, a família dos espaços de potências fracionárias geradas pelo operador $A+\bar{k} I$. 
Seja $0 \leq \alpha<1$ e seja $g: X^{\alpha} \rightarrow X$ uma função Lipschitziana em conjuntos limitados de $X^{\alpha}$. No que segue nesta seção $\pi$ denota o semifluxo local definido em $X^{\alpha}$ gerado pelas soluções da equação de evolução

$$
\dot{u}+A u=g(u)
$$

Proposição 4.1.1. Assuma a notação apresentada acima. Seja $T \in(0, \infty)$ e suponha que $u:[0, T] \rightarrow X^{\alpha}$ e $u_{k}:[0, T] \rightarrow X^{\alpha}, k \in \mathbb{N}$, sejam soluções de $\pi$. Suponha que exista uma constante $R \geq 0$ tal que

$$
|u(t)|_{X^{\alpha}} \leq R \text { e }\left|u_{k}(t)\right|_{X^{\alpha}} \leq R \text {, para todo } k \in \mathbb{N} \text { e todo } t \in[0, T]
$$

Se $\left|u_{k}(0)-u(0)\right|_{X} \rightarrow 0$, quando $k \rightarrow \infty$, então para todo $T_{0} \in(0, T]$,

$$
\left|u_{k}(t)-u(t)\right|_{X^{\alpha}} \rightarrow 0, \text { quando } k \rightarrow \infty
$$

uniformemente para $t \in\left[T_{0}, T\right]$.

Demonstração. Como $g$ é uma função Lipschitziana em conjuntos limitados de $X^{\alpha}$, se $v_{1}, v_{2} \in$ $\left\{v \in X^{\alpha} ;|v|_{X^{\alpha}} \leq R\right\}$, existe $L=L(R)>0$ tal que

$$
\left|g\left(v_{1}\right)-g\left(v_{2}\right)\right|_{X} \leq L\left|v_{1}-v_{2}\right|_{X^{\alpha}}
$$

O Lema 1.7.2 implica que para todo $k \in \mathbb{N}$

$$
u_{k}(t)-u(t)=e^{-A t}\left(u_{k}(0)-u(0)\right)+\int_{0}^{t} e^{-A(t-s)}\left[g\left(u_{k}(s)\right)-g(u(s))\right] d s
$$

Logo

$$
\begin{aligned}
\left|u_{k}(t)-u(t)\right|_{X^{\alpha}} & \leq\left|e^{-A t}\left(u_{k}(0)-u(0)\right)\right|_{X^{\alpha}}+\int_{0}^{t}\left|e^{-A(t-s)}\left(g\left(u_{k}(s)\right)-g(u(s))\right)\right|_{X^{\alpha}} d s \\
& \leq\left|A^{\alpha} e^{-A t}\right|\left|u_{k}(0)-u(0)\right|_{X}+\int_{0}^{t}\left|A^{\alpha} e^{-A(t-s)}\right|\left|g\left(u_{k}(s)\right)-g(u(s))\right|_{X} d s \\
& \leq C_{\alpha} t^{-\alpha}\left|u_{k}(0)-u(0)\right|_{X}+C_{\alpha} L \int_{0}^{t}(t-s)^{-\alpha}\left|u_{k}(s)-u(s)\right|_{X^{\alpha}} d s
\end{aligned}
$$

onde $C_{\alpha} \in[0, \infty)$ é a constante como no Teorema 1.5.22. Uma aplicação do Lema 7.1.1 em [15] 
implica que existe uma constante $C^{\prime} \in[0, \infty)$, dependendo somente de $\alpha, C_{\alpha}, L$ e $T$, tal que

$$
\left|u_{k}(t)-u(t)\right|_{X^{\alpha}} \leq C^{\prime} t^{-\alpha}\left|u_{k}(0)-u(0)\right|_{X}, \text { para todo } k \in \mathbb{N}, t \in(0, T] .
$$

Como $\left|u_{k}(0)-u(0)\right|_{X} \rightarrow 0$, quando $k \rightarrow \infty$, segue para todo $T_{0} \in(0, T]$,

$$
\left|u_{k}(t)-u(t)\right|_{X^{\alpha}} \rightarrow 0 \text {, quando } k \rightarrow \infty \text {. }
$$

Além disso, a convergência é uniforme para $t \in\left[T_{0}, T\right]$.

No próximo teorema apresentamos um resultado de compacidade para o caso em que $X$ seja um espaço de Hilbert e $A$ um operador auto-adjunto limitado inferiormente.

Suponha agora que $X$ seja um espaço de Hilbert e $A: D(A) \subset X \rightarrow X$ um operador autoadjunto limitado inferiormente. Seja $\bar{k} \geq 0$ tal que $\operatorname{re} \sigma(A+\bar{k} I)>0$ e $X^{\beta}, \beta \in \mathbb{R}$, a família de espaços de potências fracionárias gerados por $A+\bar{k} I$. Sejam $\alpha \in[0,1)$ e $g: X^{\alpha} \rightarrow X$ uma função Lipschitziana em subconjuntos limitados de $X^{\alpha}$ e $\pi$ o semifluxo local em $X^{\alpha}$ gerado pelas soluções da equação diferencial

$$
\dot{u}+A u=g(u) .
$$

Neste caso valem as propriedades demonstradas na Seção 2.2 e serão utilizadas para demonstrar o seguinte resultado.

Teorema 4.1.2. Com a notação introduzida acima, se $K \subset X^{\alpha}$ é um conjunto $\pi$-invariante e compacto, então $K \subset X^{1}=D(A)$ e $K$ é compacto em $X^{1}$.

Demonstração. Como $K$ é $\pi$-invariante, a Proposição 1.7 .4 implica que $K$ é limitado em $X^{\gamma}$ para todo $\gamma \in(\alpha, 1]$.

Afirmamos que $K$ é compacto em $X^{1}$. De fato, seja $\left(\bar{u}_{n}\right)_{n}$ uma sequência em $K$. Logo, existe uma sequência de soluções $\left(u_{n}\right)_{n}$ de $\pi$ em $K$ tais que $u_{n}(0)=\bar{u}_{n}$, para todo $n \in \mathbb{N}$.

Fixemos $0<\beta<1, \operatorname{com} \beta>\alpha$. Para todo $n \in \mathbb{N}$, $u_{n}$ é diferenciável em $X^{\beta}$ e uma aplicação 
do Teorema 1.7.6 implica que existe uma constante $C>0$ tal que

$$
\left|v_{n}\right|_{X^{\beta}} \leq C, \text { para todo } n \in \mathbb{N}
$$

onde $v_{n}:=\partial\left(u_{n} ; X^{\beta}\right)(0), n \in \mathbb{N}$, isto é, para cada $n \in \mathbb{N}, v_{n}$ é a derivada de $u_{n}$ em $X^{\beta}$ no instante $t=0$.

Como $K$ é um conjunto compacto em $X^{\alpha}$, existem uma subsequência $\left(\bar{u}_{n_{m}}\right)_{m}$ de $\left(\bar{u}_{n}\right)_{n}$ e um $\bar{u} \in K$ tais que

$$
\left|\bar{u}_{n_{m}}-\bar{u}\right|_{X^{\alpha}} \rightarrow 0, \text { quando } m \rightarrow \infty
$$

Recordemos que as fórmulas 2.2.8) e 2.2.9) implicam que $\varphi_{0, \alpha-1}(A+\bar{k} I)=A_{(1-\alpha)} \varphi_{1, \alpha} \mathrm{e}$, portanto, é uma aplicação contínua. Logo

$$
\left|\varphi_{0, \alpha-1}(A+\bar{k} I)\left(\bar{u}_{n_{m}}\right)-\varphi_{0, \alpha-1}(A+\bar{k} I)(\bar{u})\right|_{X^{\alpha-1}} \rightarrow 0, \text { quando } m \rightarrow \infty
$$

Além disso

$$
\left|g\left(\bar{u}_{n_{m}}\right)-g(\bar{u})\right|_{X} \rightarrow 0, \text { quando } m \rightarrow \infty .
$$

Portanto,

$$
\left|-\varphi_{0, \alpha-1} A \bar{u}_{n_{m}}+\varphi_{0, \alpha-1} g\left(\bar{u}_{n_{m}}\right)-\left(-\varphi_{0, \alpha-1} A \bar{u}+\varphi_{0, \alpha-1} g(\bar{u})\right)\right|_{X^{\alpha-1}} \rightarrow 0, \text { quando } m \rightarrow \infty
$$

Por outro lado,

$$
\partial\left(u_{n} ; X^{\alpha}\right)(t)=-A u_{n}(t)+g\left(u_{n}(t)\right), \text { para todo } n \in \mathbb{N} \text { e todo } t \in \mathbb{R}
$$

Aqui para cada $n \in \mathbb{N}, \partial\left(u_{n} ; X^{\alpha}\right)(t)$, denota a derivada de $u_{n}$ em $X^{\alpha}$ no instante $t$. Concluímos que

$$
\left|\varphi_{0, \alpha-1} v_{n_{m}}-\left(-\varphi_{0, \alpha-1} A \bar{u}+\varphi_{0, \alpha-1} g(\bar{u})\right)\right|_{X^{\alpha-1}} \rightarrow 0, \text { quando } m \rightarrow \infty,
$$

uma vez que $X^{\beta}$ está continuamente imerso em $X^{\alpha}$, e, portanto, $v_{n}=\partial\left(u_{n} ; X^{\alpha}\right)(0)$. Para finalizar a demonstração afirmamos que

$$
\left|v_{n_{m}}-(-A \bar{u}+g(\bar{u}))\right|_{X^{0}} \rightarrow 0, \text { quando } m \rightarrow \infty
$$


De fato, pela Desigualdade de Interpolação da Proposição 2.2.4.

$$
\begin{aligned}
\left|v_{n_{m}}-(-A \bar{u}+g(\bar{u}))\right|_{X} & \leq\left|\varphi_{\alpha, \alpha-1}\left(v_{n_{m}}+A \bar{u}-g(\bar{u})\right)\right|_{X^{\alpha-1}}^{\alpha}\left|v_{n_{m}}+A \bar{u}-g(\bar{u})\right|_{X^{\alpha}}^{1-\alpha} \\
& \leq\left|\varphi_{0, \alpha-1}\left(v_{n_{m}}+A \bar{u}-g(\bar{u})\right)\right|_{X^{\alpha-1}}^{\alpha}\left(\left|v_{n_{m}}\right|_{X^{\beta}}+|A \bar{u}+g(\bar{u})|_{X^{\alpha}}\right)^{1-\alpha} \\
& \leq \widetilde{C}\left|\varphi_{0, \alpha-1}\left(v_{n_{m}}+A \bar{u}-g(\bar{u})\right)\right|_{X^{\alpha-1}}^{\alpha},
\end{aligned}
$$

onde $\widetilde{C}$ é uma constante positiva dada por $(C+|A \bar{u}-g(\bar{u})|)^{1-\alpha}$. Logo, a convergência em (4.1.3) está demonstrada.

A convergência em (4.1.3) implica que $\left|-A \bar{u}_{n_{m}}+g\left(\bar{u}_{n_{m}}\right)-(-A \bar{u}+g(\bar{u}))\right|_{X} \rightarrow 0$ quando $m \rightarrow \infty$. Como $\left|g\left(\bar{u}_{n_{m}}\right)-g(\bar{u})\right|_{X} \rightarrow 0$ quando $m \rightarrow \infty$, temos que $\left|A \bar{u}_{n_{m}}-A \bar{u}\right|_{X} \rightarrow 0$ quando $m \rightarrow \infty$. Portanto,

$$
\left|(A+\bar{k} I) \bar{u}_{n_{m}} \rightarrow(A+\bar{k} I) \bar{u}\right|_{X} \rightarrow 0 \text { quando } m \rightarrow \infty
$$

ou seja, $\left|\bar{u}_{n_{m}}-\bar{u}\right|_{X^{1}} \rightarrow 0$ quando $m \rightarrow \infty$. Portanto, $K \subset X^{1}$ é compacto.

\subsection{Construção da função de Lyapunov}

Nesta seção vamos assumir as Hipóteses (HL1), (HL2), (HL3) e (HNL) e toda notação introduzida nos Capítulos 2 e 3. Seja $\pi$ o semifluxo definido na Seção 3.2 .

Nosso objetivo é mostrar que com as hipóteses consideradas, o semifluxo $\pi$ é gradiente. Para isso devemos exibir uma função de Lyapunov $\mathscr{L}: X^{1 / 2} \rightarrow \mathbb{R}$. Notemos que $H_{0}^{1}(\Omega)=X^{1 / 2}$.

Defina $\mathscr{L}: H_{0}^{1}(\Omega) \rightarrow \mathbb{R}$ por

$$
\mathscr{L}(u)=\frac{1}{2}\langle u, u\rangle_{H_{0}^{1}(\Omega)}-\int_{\Omega} F(x, u(x)) d x, u \in H_{0}^{1}(\Omega) .
$$

Proposição 4.2.1. Sejam $T \in(0, \infty]$ e $u:[0, T) \rightarrow H_{0}^{1}(\Omega)$ uma solução de $\pi$. Então $\mathscr{L} \circ u$ é contínua em $[0, T)$, diferenciável em $(0, T)$ e para $t \in(0, T)$

$$
(\mathscr{L} \circ u)^{\prime}(t)=-|\dot{u}(t)|_{L^{2}(\Omega)}^{2}
$$

Demonstração. Uma aplicação da Proposição 3.1 .5 nos fornece que $u \mapsto \int_{\Omega} F(x, u(x)) d x$ é 
Fréchet diferenciável. Logo $\mathscr{L}$ também é Fréchet diferenciável e

$$
D \mathscr{L}(w) \cdot v=\langle w, v\rangle_{H_{0}^{1}(\Omega)}-\int_{\Omega} \widehat{f}(w(x)) v(x) d x, \text { para } w, v \in H_{0}^{1}(\Omega) .
$$

A Proposição 3.2.2 implica que $\mathscr{L} \circ u$ é contínua em $[0, T)$ e diferenciável em $(0, T)$ e

$$
(\mathscr{L} \circ u)^{\prime}(t)=D \mathscr{L}(u(t)) \cdot \dot{u}(t)=\langle u, \dot{u}\rangle_{H_{0}^{1}(\Omega)}-\int_{\Omega} \widehat{f}(u(t)(x)) \dot{u}(t) d x \text {, para } t \in(0, T) .
$$

Suponhamos que $\bar{\rho}>2$ ou $a^{2} \notin \mathscr{E}_{1}$. Segue da Proposição 2.2.13 que

$$
\left\langle u(t), u^{\prime}(t)\right\rangle_{H_{0}^{1}(\Omega)}=\left(\mathbf{A}_{(1-\alpha)} u(t)\right) \cdot u^{\prime}(t), \text { para } t \in(0, T) .
$$

Além disso, aplicando a Proposição 3.1 .5 temos que

$$
\mathbf{f}(u(t)) \cdot u^{\prime}(t)=\int_{\Omega} \widehat{f}(u(t)(x)) \dot{u}(t)(x) d x, \text { para } t \in(0, T)
$$

Portanto, para $t \in(0, T)$ obtemos

$$
\begin{aligned}
(\mathscr{L} \circ u)^{\prime}(t) & =\left(\mathbf{A}_{(1-\alpha)} u(t)\right) \cdot \dot{u}(t)-\mathbf{f}(u(t)) \cdot \dot{u}(t) \\
& =\left(\mathbf{A}_{(1-\alpha)} u(t)-\mathbf{f}(u(t))\right) \cdot \dot{u}(t) \\
& =-\left(\varphi_{0,-\alpha} \dot{u}(t)\right) \cdot \dot{u}(t)=-\langle\dot{u}(t), \dot{u}(t)\rangle_{L^{2}(\Omega)} .
\end{aligned}
$$

Para completar a demonstração, suponha agora $\bar{\rho}=2$ e $a^{2} \in \mathscr{E}_{1}$. Então

$$
\begin{aligned}
(\mathscr{L} \circ u)^{\prime}(t) & =\langle u(t), \dot{u}(t)\rangle_{H_{0}^{1}(\Omega)}-\langle\dot{u}(t), \widehat{f}(u(t))\rangle_{L^{2}(\Omega)} \\
& =\left(\mathbf{A}_{(1-\alpha)} u(t)\right) \cdot \dot{u}(t)-\mathbf{f}(u(t)) \cdot \dot{u}(t) \\
& =-\left(\varphi_{0,-\alpha} \dot{u}(t)\right) \cdot \dot{u}(t)=-\langle\dot{u}(t), \dot{u}(t)\rangle_{L^{2}(\Omega)} .
\end{aligned}
$$

A demonstração está completa.

Segue da Proposição 4.2.1 que $\mathscr{L}$ é uma função de Lyapunov para o semifluxo $\pi$. Mais precisamente:

Corolário 4.2.2. $\pi$ é um semifluxo global em $Y=H_{0}^{1}(\Omega)$ e satisfaz as seguintes propriedades:

(a) todo conjunto limitado de Y é eventualmente limitado; 
(b) o conjunto de equilíbrio de $\pi$ é limitado;

(c) existe $\mathscr{L}: Y \rightarrow \mathbb{R}$ contínua, limitada inferiormente, não-crescente sobre soluções de $\pi$ e sempre que $\mathscr{L}(x \pi t)=\mathscr{L}(x)$, para todo $t \in[0, \infty)$, então $x$ é ponto de equilíbrio.

Demonstração. As Proposições 3.1.5 e 3.2.2 mostram que $\mathscr{L}$ é contínua e não-crescente sobre soluções de $\pi$. Seja $u_{0} \in H_{0}^{1}(\Omega)$ e escreva $u(t)=u_{0} \pi t$, para $t \in\left[0, \omega_{u_{0}}\right)$. Temos que $\mathscr{L}(u(t)) \leq$ $\mathscr{L}\left(u_{0}\right)$, ou seja,

$$
\frac{1}{2}\langle u(t), u(t)\rangle_{H_{0}^{1}(\Omega)}-\int_{\Omega} F(x, u(t)(x)) d x \leq \frac{1}{2}\left\langle u_{0}, u_{0}\right\rangle_{H_{0}^{1}(\Omega)}-\int_{\Omega} F\left(x, u_{0}(x)\right) d x, t \in\left[0, \omega_{u_{0}}\right) .
$$

Logo,

$$
\begin{aligned}
\frac{1}{2}\langle u(t), u(t)\rangle_{H_{0}^{1}(\Omega)} & \leq \frac{1}{2}\left\langle u_{0}, u_{0}\right\rangle_{H_{0}^{1}(\Omega)}-\int F\left(x, u_{0}(x)\right) d x_{\Omega}+\int_{\Omega} F(x, u(t)(x)) d x \\
& \leq \mathscr{L}\left(u_{0}\right)+\int_{\Omega} c(x) d x, t \in\left[0, \omega_{u_{0}}\right) .
\end{aligned}
$$

Portanto, temos que toda solução de $\pi$ é limitada em $H_{0}^{1}(\Omega)$ e segue da Proposição 1.7 .3 que $\pi$ é um semifluxo global e, portanto, $\pi$ não explode em subconjuntos limitados de $H_{0}^{1}(\Omega)$. Da mesma maneira, segue que todo subconjunto $B$ limitado em $H_{0}^{1}(\Omega)$ é $\pi$-eventualmente limitado, tomando $t_{B}=0$. Também dado $u \in H_{0}^{1}(\Omega)$ temos

$$
\mathscr{L}(u)=\frac{1}{2}\langle u, u\rangle_{H_{0}^{1}(\Omega)}-\int_{\Omega} F(x, u(x)) d x \geq-\int_{\Omega} F(x, u(x)) d x \geq-\int_{\Omega} c(x) d x,
$$

ou seja, $\mathscr{L}$ é limitado inferiormente.

Seja $u:[0, \infty) \rightarrow H_{0}^{1}(\Omega)$ uma solução de $\pi$ sobre a qual $\mathscr{L}$ é constante. A Proposição 4.2.1 implica que $\dot{u}(t)=0$, para todo $t>0$ e, portanto, $u(t)$ é uma solução constante. Logo $u(0)$ é um ponto de equilíbrio de $\pi$.

Finalmente, seja $u_{0}$ um ponto de equilíbrio de $\pi$. Suponha $\bar{\rho}>2$ ou $a^{2} \notin \mathscr{E} 1$. Então $\mathbf{A}_{(1-\alpha)}\left(u_{0}\right)$ está definido e $\mathbf{A}_{(1-\alpha)}\left(u_{0}\right)=\mathbf{f}\left(u_{0}\right)$. Portanto,

$$
\begin{aligned}
\left\langle u_{0}, u_{0}\right\rangle_{H_{0}^{1}(\Omega)} & =\left(\mathbf{A}_{(1-\alpha)} u_{0}\right) \cdot u_{0}=\mathbf{f}\left(u_{0}\right) \cdot u_{0} \\
& =\int_{\Omega} f\left(x, u_{0}(x)\right) u_{0}(x) d x \leq \int_{\Omega} c(x) d x<\infty .
\end{aligned}
$$

Logo, o conjunto dos equilíbrios de $\pi$ é limitado em $H_{0}^{1}(\Omega)$. Um argumento similar mostra 
que se $\bar{\rho}=2$ e $a^{2} \in \mathscr{E}_{1}$, temos novamete que o conjunto dos equilíbrios de $\pi$ é limitado em $H_{0}^{1}(\Omega)$.

\subsection{Estimativas de truncamento}

Nesta seção vamos assumir as Hipóteses (HL1), (HL2), (HL3) e (HNL) e toda notação introduzida nos Capítulos 2 e 3. Seja $\pi$ o semifluxo definido na Seção 3.2 .

Passamos agora para a estimativas de truncamento. Seja $\bar{\phi}: \mathbb{R} \rightarrow[0,1]$ uma função de classe $C^{1} \operatorname{com} \bar{\phi}(s)=0$ para $s \in(-\infty, 1]$ e $\bar{\phi}(s)=1$ para $s \in[2, \infty)$. Seja $\phi:=\bar{\phi}^{2}$. Para $k \in \mathbb{N}$ defina a função $\bar{\phi}_{k}: \mathbb{R}^{N} \rightarrow \mathbb{R}$ e $\phi_{k}: \mathbb{R}^{N} \rightarrow \mathbb{R}$ por

$$
\bar{\phi}_{k}(x)=\bar{\phi}\left(|x|^{2} / k^{2}\right) \quad \text { e } \quad \phi_{k}(x)=\phi\left(|x|^{2} / k^{2}\right), x \in \mathbb{R}^{N} \text {. }
$$

Defina também

$$
C_{\phi}=2 \sqrt{2} \sup _{y \in \mathbb{R}}\left|\phi^{\prime}(y)\right| \quad \text { e } \quad C_{\bar{\phi}}=2 \sqrt{2} \sup _{y \in \mathbb{R}}\left|\bar{\phi}^{\prime}(y)\right|
$$

e seja $\kappa \in\left(0, \lambda_{1}\right)$ arbitrário, onde $\lambda_{1}$ é como definido na Hipótese (HL3). Para cada $k \in \mathbb{N}$, sejam

$$
b_{k}=\left(\frac{a_{1}\left(C_{\bar{\phi}}\right)^{2}}{k^{2}}\right) \quad \text { e } \quad c_{k}=\int_{\Omega} \phi_{k}(x) c(x) d x .
$$

Lema 4.3.1. As seguintes estimativas são válidas:

$$
\sup _{x \in \Omega}\left|\nabla \phi_{k}(x)\right| \leq C_{\phi} / k \quad e \quad \sup _{x \in \Omega}\left|\nabla \bar{\phi}_{k}(x)\right| \leq C_{\bar{\phi}} / k
$$

Demonstração. Observemos que para $x \in \mathbb{R}^{N}$,

$$
\nabla \phi_{k}(x)=\left(\frac{2}{k^{2}}\right) \phi^{\prime}\left(\frac{|x|^{2}}{k^{2}}\right) x \quad \text { e } \quad \nabla \bar{\phi}_{k}(x)=\left(\frac{2}{k^{2}}\right) \bar{\phi}^{\prime}\left(\frac{|x|^{2}}{k^{2}}\right) x
$$

Como $\operatorname{supp}\left(\phi^{\prime}\right) \subseteq[1,2]$, segue que

$$
\sup _{x \in \Omega}\left|\nabla \phi_{k}(x)\right| \leq \frac{2}{k^{2}} \sup _{|x| \leq \sqrt{2} k} \phi^{\prime}\left(\frac{|x|^{2}}{k^{2}}\right)|x|=C_{\phi} / k .
$$


Analogamente mostramos a segunda desigualdade.

Temos a seguinte estimativa importante para o resultado de existência de atratores.

Teorema 4.3.2. Sejam $R \geq 0, \tau>0$ e $u:[0, \infty) \rightarrow H_{0}^{1}(\Omega)$ solução de $\pi$ tal que $|u(t)|_{H_{0}^{1}(\Omega)} \leq R$, para todo $t \in[0, \tau)$. Então para todo $t \in[0, \tau)$

$$
\int_{\Omega} \phi_{k}(x)|u(t)(x)|^{2} d x \leq R^{2} e^{-2 \kappa t}+\frac{b_{k} R^{2}+c_{k}}{\kappa} .
$$

Demonstração. Afirmamos que para todo $u \in D(A)=X^{1} \subset H_{0}^{1}(\Omega)$,

$$
\left(-\mathbf{A}_{(1-\alpha)} u\right) \cdot\left(\phi_{k} u\right)+\kappa \int_{\Omega} \phi_{k}(x)|u(x)|^{2} d x \leq \int_{\Omega} a_{1} C_{\bar{\phi}}^{2} k^{-2}|u|^{2} d x .
$$

Suponhamos primeiro que $\bar{\rho}>2$ ou $a^{2} \notin \mathscr{E}_{1}$. De fato, para $u \in X^{1}$, temos

$$
\begin{aligned}
\left(-\mathbf{A}_{(1-\alpha)} u\right) \cdot\left(\phi_{k} u\right) & =\left(-\varphi_{0,-\alpha} \mathbf{A} \varphi_{1,1-\alpha}^{-1} u\right) \cdot\left(\phi_{k} u\right) \\
& =\left(-\varphi_{0,-\alpha} \mathbf{A} u\right) \cdot\left(\phi_{k} u\right)=\left\langle-\mathbf{A} u, \phi_{k} u\right\rangle_{L^{2}(\Omega)} .
\end{aligned}
$$

Se $\bar{\rho}=2$ e $a^{2} \in \mathscr{E} 1$, então lembremos que $\alpha=0$ e dessa forma

$$
\left(-\mathbf{A}_{(1-\alpha)} u\right) \cdot\left(\phi_{k} u\right)=\left\langle-\mathbf{A}_{(1-\alpha)} u, \phi_{k} u\right\rangle_{X}=\left\langle-\mathbf{A} u, \phi_{k} u\right\rangle_{L^{2}(\Omega)} \cdot
$$

Notemos também que

$$
\begin{aligned}
\left\langle L u-\beta u, \phi_{k} u\right\rangle_{L^{2}(\Omega)} & =\int_{\Omega}\left[\sum_{i, j} a_{i j}(x) \partial_{i} u(x) \partial_{j} \phi_{k} u(x)-\beta(x) u(x) \phi_{k} u(x)\right] d x \\
\mathbf{A} \nabla u . \nabla \phi_{k} u & =\sum_{i, j} a_{i j}(x) \partial_{i} u(x) \partial_{j} \phi_{k} u(x) .
\end{aligned}
$$

Portanto,

$$
\begin{aligned}
\left(-\mathbf{A}_{(1-\alpha)} u\right) & .\left(\phi_{k} u\right)+\kappa \int_{\Omega} \phi_{k}(x)|u(x)|^{2} d x=\int_{\Omega}(-\mathbf{A} u(x)) \phi_{k}(x) u(x)+\kappa \int_{\Omega} \phi_{k}(x)|u(x)|^{2} d x \\
& =\int_{\Omega}(L u)(x)\left(\phi_{k} u\right)(x)-\beta(x) u(x) \phi_{k}(x) u(x) d x+\kappa \int_{\Omega} \phi_{k}(x)|u(x)|^{2} d x \\
& =\int_{\Omega}(A \nabla u)(x)\left(\nabla \phi_{k} u\right)(x) d x-(\beta(x)-\kappa) \int_{\Omega}\left|\bar{\phi}_{k} u(x)\right|^{2} d x \\
& =\int_{\Omega} \sum_{i, j} a_{i j}(x) \partial_{i} u(x) \partial_{j} \phi_{k} u(x) d x-(\beta(x)-\kappa) \int_{\Omega}\left|\bar{\phi}_{k} u(x)\right|^{2} d x .
\end{aligned}
$$


Observemos que

$$
\partial_{j} \phi_{k} u=\partial_{j}\left(\bar{\phi}_{k}^{2} u\right)=2 u \bar{\phi}_{k} \partial_{j} \bar{\phi}_{k}+\phi_{k} \partial_{j} u
$$

$\mathrm{e}$

$$
\partial_{i}\left(\bar{\phi}_{k} u\right) \partial_{j}\left(\bar{\phi}_{k} u\right)=|u|^{2} \partial_{i} \bar{\phi}_{k} \partial_{j} \bar{\phi}_{k}+2 u \bar{\phi}_{k} \partial_{i} \bar{\phi}_{k} \partial_{j} u+\phi_{k} \partial_{i} u \partial_{j} u
$$

Fórmula 4.3.5 implica que

$$
\begin{aligned}
\left(-\mathbf{A}_{(1-\alpha)} u\right) \cdot\left(\phi_{k} u\right) & +\kappa \int_{\Omega} \phi_{k}(x)|u(x)|^{2} d x= \\
& -\int_{\Omega}\left(\sum_{i, j} 2 u \bar{\phi}_{k} a_{i j} \partial_{i} u \partial_{j} \bar{\phi}_{k}+\sum_{i, j} \phi_{k} a_{i j} \partial_{i} u \partial_{j} u\right) d x \\
& -(\beta(x)-\kappa) \int_{\Omega}\left|\bar{\phi}_{k} u(x)\right|^{2} d x
\end{aligned}
$$

Somando e subtraindo o termo $|u|^{2} \sum_{i, j} a_{i j} \partial_{i} \bar{\phi}_{k} \partial_{j} \bar{\phi}_{k}$ na integral em 4.3.7) e utilizando igualdade 4.3.6, obtemos

$$
\begin{aligned}
\left(-\mathbf{A}_{(1-\alpha)} u\right) . & \left(\phi_{k} u\right)+\kappa \int_{\Omega} \phi_{k}(x)|u(x)|^{2} d x=\int_{\Omega} \sum_{i, j} a_{i j}|u|^{2} \partial_{i} \bar{\phi}_{k} \partial_{j} \bar{\phi}_{k} \\
& -\left[\int_{\Omega} \sum_{i, j} a_{i j}(x) \partial_{i}\left(\bar{\phi}_{k} u\right)(x) \partial_{j}\left(\bar{\phi}_{k} u\right)(x) d x+(\beta(x)-\kappa) \int_{\Omega}\left|\bar{\phi}_{k} u(x)\right|^{2} d x\right] .
\end{aligned}
$$

Notemos que o Lema 2.3.1 implica que

$$
\int_{\Omega} \sum_{i, j} a_{i j}(x) \partial_{i}\left(\bar{\phi}_{k} u\right)(x) \partial_{j}\left(\bar{\phi}_{k} u\right)(x) d x+(\beta(x)-\kappa) \int_{\Omega}\left|\bar{\phi}_{k} u(x)\right|^{2} d x \geq 0
$$

Logo,

$$
\left(-\mathbf{A}_{(1-\alpha)} u\right) \cdot\left(\phi_{k} u\right)+\kappa \int_{\Omega} \phi_{k}(x)|u(x)|^{2} d x \leq \int_{\Omega}|u|^{2}\left(A \nabla \bar{\phi}_{k}\right) . \nabla \bar{\phi}_{k} d x,
$$

concluindo a demonstração da desigualdade 4.3.2.

Para cada $k \in \mathbb{N}$ defina a função $V_{k}: H_{0}^{1}(\Omega) \rightarrow \mathbb{R}$ por

$$
V_{k}(u)=\frac{1}{2} \int_{\Omega} \phi_{k}(x)|u(x)|^{2} d x, u \in H_{0}^{1}(\Omega) .
$$

Temos que $V_{k}$ é Fréchet diferenciável e

$$
D V_{k}(u) v=\int_{\Omega} \phi_{k}(x) u(x) v(x) d x, u, v \in H_{0}^{1}(\Omega)
$$


Seja $u:[0, \infty) \rightarrow H_{0}^{1}(\Omega)$ uma solução de $\pi$. Pela Proposição 3.2.2 temos que $V_{k} \circ u$ é diferenciável em $(0, \infty)$ e, para $t \in(0, \infty)$, temos

$$
\begin{aligned}
\left(V_{k} \circ u\right)^{\prime}(t)=D V_{k}(u(t)) \dot{u}(t) & =\int_{\Omega} \phi_{k}(x) u(t)(x) \dot{u}(t)(x) d x \\
& =\left\langle\dot{u}(t), \phi_{k} u(t)\right\rangle_{L^{2}(\Omega)} .
\end{aligned}
$$

Como $\phi_{k} u(t) \in H_{0}^{1}(\Omega)=X^{1 / 2} \subset X^{\alpha}$, segue que para $t \in(0, \infty)$

$$
\left\langle\dot{u}(t), \phi_{k} u(t)\right\rangle_{L^{2}(\Omega)}=\left(\varphi_{0,-\alpha} \dot{u}(t)\right) \cdot\left(\phi_{k} u(t)\right)=\left(-\mathbf{A}_{(1-\alpha)} u(t)+\mathbf{f}(u(t))\right) \cdot\left(\phi_{k} u(t)\right) .
$$

Portanto,

$$
\begin{aligned}
\left(V_{k} \circ u\right)^{\prime}(t) & +2 \kappa\left(V_{k} u\right)(t)=\left(-\mathbf{A}_{(1-\alpha)} u(t)\right) \cdot\left(\phi_{k} u(t)\right) \\
& +\int_{\Omega} \widehat{f}(u(t))(x) \phi_{k}(x) u(t)(x) d x+\kappa \int_{\Omega} \phi_{k}(x)|u(t)(x)|^{2} d x \\
& \leq\left(-\mathbf{A}_{(1-\alpha)} u(t)\right)\left(\phi_{k} u(t)\right)+\kappa \int_{\Omega} \phi_{k}(x)|u(t)(x)|^{2} d x+\int_{\Omega} \phi_{k}(x) c(x) d x \\
& \leq \int_{\Omega} a_{1} C_{\frac{1}{\phi}}^{2} k^{-2}|u(t)|^{2} d x+\int_{\Omega} \phi_{k}(x) c(x) d x .
\end{aligned}
$$

Logo, sempre que $R \geq 0, \tau \in(0, \infty)$ e $|u(t)|_{H_{0}^{1}} \leq R$ para todo $t \in[0, \tau]$, temos

$$
\left(V_{k} \circ u\right)^{\prime}(t)+2 \kappa\left(V_{k} \circ u\right)(t) \leq b_{k} R^{2}+c_{k}, t \in(0, \tau] .
$$

Disto segue que

$$
V_{k}(u(t)) \leq e^{-2 \kappa t} V_{k}(u(t))+b_{k}\left(R^{2}+c_{k}\right) / 2 \kappa, \text { para todo } t \in[0, \tau],
$$

ou seja,

$$
\int_{\Omega} \phi_{k}(x)|u(t)(x)|^{2} d x \leq e^{-2 \kappa} R^{2}+b_{k}\left(R^{2}+c_{k}\right) / \kappa, \text { para todo } t \in[0, \tau] \text {. }
$$

A demonstração está completa. 


\subsection{Compacidade assintótica e existência de atrator}

Podemos demonstrar agora que $\pi$ satisfaz a condição $(a)$ do Teorema 1.6 .8 de existência de atratores.

Teorema 4.4.1. O semifluxo $\pi$ é assintoticamente compacto.

Demonstração. Sejam $B$ um subconjunto eventualmente limitado em $Y=H_{0}^{1}(\Omega),\left(v_{n}\right)_{n}$ uma sequência em $B$ e $\left(t_{n}\right)_{n}$ uma sequência em $[0, \infty) \operatorname{com} t_{n} \rightarrow \infty$. Devemos mostrar a existência de uma subsequência de $\left(v_{n} \pi t_{n}\right)_{n}$ convergente em $H_{0}^{1}(\Omega)$.

Como $B$ é eventualmente limitado existem $t_{B} \in[0, \infty)$ e $R \geq 0$ tais que

$$
|v \pi t|_{H_{0}^{1}(\Omega)} \leq R, \text { para todo } v \in B \text { e } t \geq t_{B}
$$

Podemos assumir, sem perda de generalidade, que $t_{n} \geq t_{B}+1$, para todo $n \in \mathbb{N}$.

Para cada $n \in \mathbb{N}$, defina $s_{n}:=t_{n}-t_{B}$ e seja $u_{n}:[0, \infty) \rightarrow H_{0}^{1}(\Omega)$ dado por $u_{n}(s)=v_{n} \pi\left(t_{B}+s\right)$, $s \geq 0$. Para cada $n \in \mathbb{N}, \tau_{n}:=s_{n}-1 \geq 0$ e $u_{n}$ é uma solução de $\pi$ com

$$
\left|u_{n}(s)\right|_{H_{0}^{1}(\Omega)} \leq R, \text { para todo } s \in[0, \infty)
$$

e $u_{n}\left(s_{n}\right)=v_{n} \pi t_{n}$

Afirmamos que existem uma sequência crescente $\left(n_{m}\right)_{m}$ em $\mathbb{N}$ e um $v \in H_{0}^{1}(\Omega)$ tais que $\left(u_{n_{m}}\left(\tau_{n_{m}}\right)\right)_{m}$ converge para $v$ em $L^{2}(\Omega)$.

Considere $\beta_{K}$ a medida de não-compacidade de Kuratowski em $X=L^{2}(\Omega)$ como na Seção 1.3.1. Para todo $k \in \mathbb{N}$ e $n_{0} \in \mathbb{N}$, temos

$$
\begin{aligned}
\beta_{K}\left(\left\{u_{n}\left(\tau_{n}\right) \mid n \in \mathbb{N}\right\}\right) & \leq \beta_{K}\left(\left\{\left(1-\bar{\phi}_{k}\right) u_{n}\left(\tau_{n}\right) \mid n \in \mathbb{N}\right\}\right)+\beta_{K}\left(\left\{\bar{\phi}_{k} u_{n}\left(\tau_{n}\right) \mid n \in \mathbb{N}\right\}\right) \\
& =\beta_{K}\left(\left\{\left(1-\bar{\phi}_{k}\right) u_{n}\left(\tau_{n}\right) \mid n \in \mathbb{N}\right\}\right)+\beta_{K}\left(\left\{\bar{\phi}_{k} u_{n}\left(\tau_{n}\right) \mid n \geq n_{0}\right\}\right) .
\end{aligned}
$$

Seja $\varepsilon>0$. Como $\tau_{n} \rightarrow \infty$ quando $n \rightarrow \infty$, podemos escolher $n_{0} \in \mathbb{N}$ tal que

$$
R^{2} e^{-2 \kappa \tau_{n}}<\varepsilon / 2, \text { para } n \geq n_{0}
$$


Além disso podemos escolher um $k_{0}>0$ tal que

$$
b_{k_{0}} R^{2}+c_{k_{0}}<\varepsilon \kappa / 2 .
$$

Segue do Teorema 4.3.2 e das desigualdades (4.4.1) e (4.4.2) que

$$
\left|\bar{\phi}_{k_{0}} u_{n}\left(\tau_{n}\right)\right|_{L^{2}(\Omega)}=\int_{\Omega} \phi_{k_{0}}(x)\left|u_{n}\left(\tau_{n}\right)(x)\right|^{2} d x<\varepsilon, \text { para } n \geq n_{0} .
$$

Logo, mostramos que para cada $\varepsilon>0$, existe um $k_{0} \in \mathbb{N}$ tal que

$$
\beta_{K}\left\{u_{n}\left(\tau_{n}\right) \mid n \in \mathbb{N}\right\} \leq \beta_{K}\left\{\left(1-\bar{\phi}_{k_{0}}\right) u_{n}\left(\tau_{n}\right) \mid n \in \mathbb{N}\right\}+\varepsilon
$$

Como $1-\bar{\phi}_{k} \in C_{0}^{1}\left(\mathbb{R}^{N}\right)$, a aplicação $u \mapsto\left(1-\bar{\phi}_{k}\right) u$ é compacta de $H_{0}^{1}(\Omega)$ em $L^{2}(\Omega)$, para todo $k \in \mathbb{N}, \operatorname{logo} \beta_{K}\left\{\left(1-\bar{\phi}_{k}\right) u_{n}\left(\tau_{n}\right) \mid n \in \mathbb{N}\right\}=0$. Portanto,

$$
\beta_{K}\left\{u_{n}\left(\tau_{n}\right) \mid n \in \mathbb{N}\right\} \leq \varepsilon, \text { para todo } \varepsilon>0
$$

o que implica que $\beta_{K}\left\{u_{n}\left(\tau_{n}\right) \mid n \in \mathbb{N}\right\}=0$. Segue da Proposição 1.3.1 que $\left\{u_{n}\left(\tau_{n}\right) \mid n \in \mathbb{N}\right\}$ é um subconjunto relativamente compacto de $L^{2}(\Omega)$. Logo existe uma sequência crescente $\left(n_{m}\right)_{m}$ em $\mathbb{N}$ e existe um $v \in L^{2}(\Omega)$ tais que

$$
\left|u_{n_{m}}\left(\tau_{n_{m}}\right)-v\right|_{L^{2}(\Omega)} \rightarrow 0 \text {, quando } m \rightarrow \infty
$$

Para completar a demonstração da afirmativa resta mostrarmos que $v \in H_{0}^{1}(\Omega)$. Note que a sequência $\left(u_{n_{m}}\left(\tau_{n_{m}}\right)\right)_{m}$ é limitada em $H_{0}^{1}(\Omega)$, e, tomando uma sequência de $\left(u_{n_{m}}\left(\tau_{n_{m}}\right)\right)_{m}$ se necessário, podemos supor que existe um $w \in H_{0}^{1}(\Omega)$ tal que $\left(u_{n_{m}}\left(\tau_{n_{m}}\right)\right)_{m}$ converge fracamente em $H_{0}^{1}(\Omega)$ para $w$. Logo, $u_{n_{m}}\left(\tau_{n_{m}}\right) \rightarrow w$, quando $m \rightarrow \infty$, em $L^{2}(\Omega)$ e pela unicidade do limite fraco e forte, segue que $v=w \in H_{0}^{1}(\Omega)$.

A fim de concluir a demonstração do teorema vamos considerar dois casos. Suponhamos, primeiramente, que $\bar{\rho}>2$ ou $a^{2} \notin \mathscr{E} 1$.

Defina $\widetilde{u}_{n}=\varphi_{1 / 2,-\alpha} \circ u_{n}$, para $n \in \mathbb{N}$. Então $\widetilde{u}_{n}$ é uma solução de $\widetilde{\pi}$ e a sequência $\left(\widetilde{u}_{n_{m}}\left(\tau_{n_{m}}\right)\right)_{m}$ converge para $\widetilde{v}:=\varphi_{1 / 2,-\alpha}(v)$ em $\varphi_{0,-\alpha}(X)$. Logo, a sequência $\left(\tilde{u}_{n_{m}}\left(\tau_{n_{m}}\right)\right)_{m}$ converge para $\tilde{v}$ em $X^{-\alpha}$. 
Pela Proposição 4.1 .1 segue que $\left(\widetilde{u}_{n_{m}}\left(\tau_{n_{m}}+1\right)\right)_{m}$ converge para $\widetilde{v} \tilde{\pi} 1$ em $\varphi_{1 / 2,-\alpha}\left(X^{1 / 2}\right)$. Como $\varphi_{1 / 2,-\alpha}\left(X^{1 / 2}\right)=X_{\widetilde{A}_{(-\alpha)}}^{1 / 2+\alpha}$ e $\varphi_{1 / 2,-\alpha}$ é contínua, segue que $\left(u_{n_{m}}\left(s_{n_{m}}\right)\right)_{m}$ converge para $v \pi 1$ em $X^{1 / 2}$, completando a prova neste caso.

Finalmente, suponha que $\bar{\rho}=2$ e $a^{2} \in \mathscr{E}_{1}$. Neste caso existe uma constante $M \geq 0$ tal que $\left|u_{n_{m}}\left(\tau_{n_{m}}+t\right)\right|_{H_{0}^{1}(\Omega)} \leq M$ e $|v \pi t|_{H_{0}^{1}} \leq M$, para todo $t \in[0, T]$. Logo, $\left|u_{n_{m}}\left(\tau_{n_{m}}\right)-v \pi 0\right|_{L^{2}(\Omega)} \rightarrow 0$ quando $m \rightarrow \infty$ e segue da Proposição 4.1.1 que $\left|u_{n_{m}}\left(\tau_{n_{m}}+1\right)-v \pi 1\right|_{L^{2}(\Omega)} \rightarrow 0$ quando $m \rightarrow \infty$. Logo, a prova está completa para este caso.

Agora podemos provar o resultado final.

Teorema 4.4.2. O semifluxo global $\pi$ possui um atrator global $\mathscr{A}$. Além disso, $\mathscr{A}$ está em $X^{1-\alpha}$ e é um conjunto compacto na norma de $X^{1-\alpha}$.

Demonstração. O Corolário 4.2.2 e o Teorema 4.4.1 implicam que as hipóteses do Teorema 1.6 .8 estão satifeitas. Portanto, $\pi$ possui um atrator global $\mathscr{A}$.

Se $\bar{\rho}=2$ e $a^{2} \in \mathscr{E}_{1}$, como $\alpha=0$ neste caso, Teorema 4.1.2 implica a segunda parte do teorema.

Se $\bar{\rho}>2$ ou $a^{2} \notin \mathscr{E} 1$, como $\pi$ é um semifluxo global segue que $\tilde{\pi}$ é um semifluxo global em $\varphi_{1 / 2,-\alpha}\left(X^{1 / 2}\right)$ e também possui um atrator global $\widetilde{\mathscr{A}}:=\varphi_{1 / 2,-\alpha}(\mathscr{A})$. Portanto, segue do Teorema 4.1.2 que $\widetilde{\mathscr{A}}$ está em $\varphi_{1-\alpha,-\alpha}\left(X^{1-\alpha}\right)$ e é compacto em $\varphi_{1-\alpha,-\alpha}\left(X^{1-\alpha}\right)$. A prova da segunda parte do teorema para este caso está completa. 


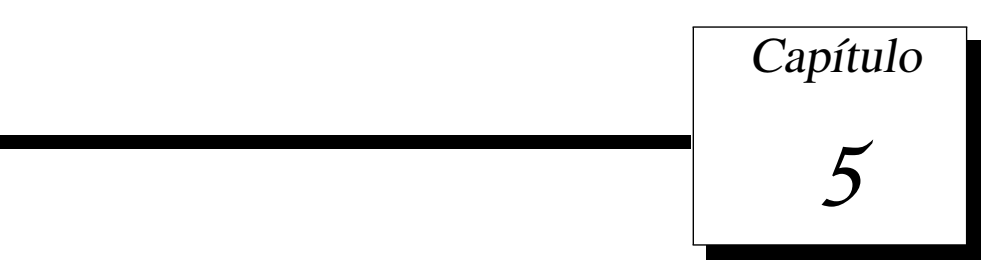

\section{Considerações finais}

Nosso trabalho de mestrado teve como finalidade entender os conceitos e técnicas desenvolvidas para demonstrar a existência de atratores globais para uma classe de equações parabólicas. Baseamos nosso trabalho no artigo [21], que é o primeiro de uma sequência de três artigos dos autores Prizzi e Rybakowski. Em todos as equações diferencias parciais consideradas são definidas em domínios arbitrários.

Em [20], os autores consideram a seguinte classe de equações das ondas amortecidas

$$
\begin{array}{ll}
\varepsilon u_{t t}+\alpha(x) u_{t}+\beta(x) u-\sum_{i j} \partial_{i}\left(a_{i j}(x) \partial_{j} u\right)=f(x, u), & t \geq 0, x \in \Omega, \\
u(x, t)=0, & t \geq 0, x \in \partial \Omega,
\end{array}
$$

onde $N \in \mathbb{N}, \Omega$ é um conjunto aberto em $\mathbb{R}^{N}$ arbitrário, $\varepsilon>0$ e $\alpha, \beta: \Omega \rightarrow \mathbb{R}$ e $f: \Omega \times \mathbb{R} \rightarrow \mathbb{R}$ são funções dadas. Em [20] mostram, para cada $\varepsilon>0$, a existência de atrator global $\mathscr{A}_{\varepsilon}$ (em $\left.H_{0}^{1}(\Omega) \times L^{2}(\Omega)\right)$ para a equação $\left(D W_{\varepsilon}\right)$. Novamente nesse trabalho a técnica de trucamento como apresentada em [21] foi utilizada. Um fato importante obtido nas demonstrações apresentadas em [20] é que as estimativas para a solução $u(t, x)$ de $\left(D W_{\varepsilon}\right)$ são uniformes com relação ao parâmetro $\varepsilon>0$.

Obsversamos que para $N=3$ e $\alpha \equiv 1$ a equação $\left(D W_{\varepsilon}\right)$ possui um problema parabólico limite que é o problema estudado neste trabalho. Como demonstrado em [21], e exposto aqui, o problema limite possui um atrator global $\widetilde{\mathscr{A}}_{0}$ em $H_{0}^{1}(\Omega)$. Em [19] os autores mostram que $\widetilde{\mathscr{A}}_{0}$ pode ser mergulhado em um conjunto compacto $\mathscr{A}_{0} \subset H_{0}^{1}(\Omega) \times L^{2}(\Omega)$ e que a família de atratores $\left(\mathscr{A}_{\varepsilon}\right)_{\varepsilon \in[0, \infty)}$ é semi-contínua superiormente quando $\varepsilon \rightarrow 0$. 


\section{Referências Bibliográficas}

[1] J.M. Arrieta, J.W. Cholewa, T. Dłtoko e A. Rodrigues-Benal, Asymptotoic behavior and attractors for reaction-diffusion equations in unbounded domains, Nonlinear Anal. 56 (2004), 515-554.

[2] A.V. Babin e M.I. Vishik, Attractor of Evolution Equations, North Holland, Amsterdam, 1991.

[3] A.V. Babin e M.I. Vishik, Attractor of partial differential evolution equations in an unbounded domain, Proc. Roy. Soc. Edinburgh Sect. A 116 (1990), 221-243.

[4] H. Brézis, Análisis Funcional, Alianza Universidad Textos 88, Madrid, 1984.

[5] A.N. Carvalho, Sistemas Dinâmicos não Lineares, Notas de Aula. Modelo de documento digital disponível em < http://www.icmc.usp.br/^andcarva/SDNL2009.pdf>. Acesso em: 13 de março de 2012.

[6] T. Cazenave e A. Haraux, An Introduction to Semilinear Evolution Equations, Oxford Science Publications, Oxford, 1998.

[7] J.W. Cholewa e T. Dłtoko, Global Attractors in Abstract Parabolic Problems, Cambridge University Press, Cambridge, 2000.

[8] R. Czaja, Differential Equations with Sectorial Operator, Wydawnictwo Uniwersytetu Slaskiego, Katowice, 2002.

[9] R. Dautray e J-L. Lions, Mathematical Analysis and Numerical Methods for Science and Technology, Volume 2, Springer-Verlag Berlin Heiddelberg, 1990.

[10] R. Dautray e J-L. Lions, Mathematical Analysis and Numerical Methods for Science and Technology, Volume 3, Springer-Verlag Berlin Heiddelberg, 1990. 
[11] K. Deimling, Nonlinear Functional Analysis, Springer-Verlag, 1985.

[12] D.G. de Figueiredo, The Ekeland Variational Principle with Applications and Detours, Tata Institute of Fundamental Research, 1989.

[13] A. Friedman, Partial Differential Equations, Holt, Rinehart and Winston, New York, 1969.

[14] J. Hale, Asymptotic Behavior of Dissipative Systems, American Mathematical Society, Providence, 1988.

[15] D. Henry, Geometric Theory of Semilinear Parabolic Equations, Springer-Verlag, Berlin, 1981.

[16] T. Kato, Perturbation Theory for Linear Operators, Springer-Verlag Berlin, 1995.

[17] O. Ladyzenskaya, Attractors for Semigroups and Evolution Equations, Cambridge University Press, Cambridge, 1991.

[18] M. Miklavcic, Stability for semilinear parabolic equations with noninvertible linear operator, Pacific Journal of Mathematics 118 (1985), 199-214.

[19] M. Prizzi e K. P. Rybakowski, Attractors for singularly perturbed damped wave equations on arbitrary unbounded domains, Top. Meth. in Nonlinear Anal. 32 (2008), 1-20.

[20] M. Prizzi e K. P. Rybakowski, Attractors for semilinear damped wave equations on arbitrary unbounded domains, Top. Meth. in Nonlinear Anal. 31 (2008), 49-82.

[21] M. Prizzi e K. P. Rybakowski, Attractors for reaction-diffusion equations on arbitrary unbounded domains, Top. Meth. in Nonlinear Anal. 30 (2007), 251-277.

[22] K.P. Rybakowski, The Homotopy Index and Partial Differential Equations, SpringerVerlag, Berlin, 1987.

[23] R. Temam, Infinite Dimensional Dynamical Systems in Mechanics and Physics, SpringerVerlag, New York, 1997.

[24] B. Wang, Attractors for reaction-diffusion equations in unbounded domains, Physica D 179 (1999), 41-52. 